\title{
La transmission des savoirs à l'ère numérique
}

\section{Arnaud Dhermy (dir.)}

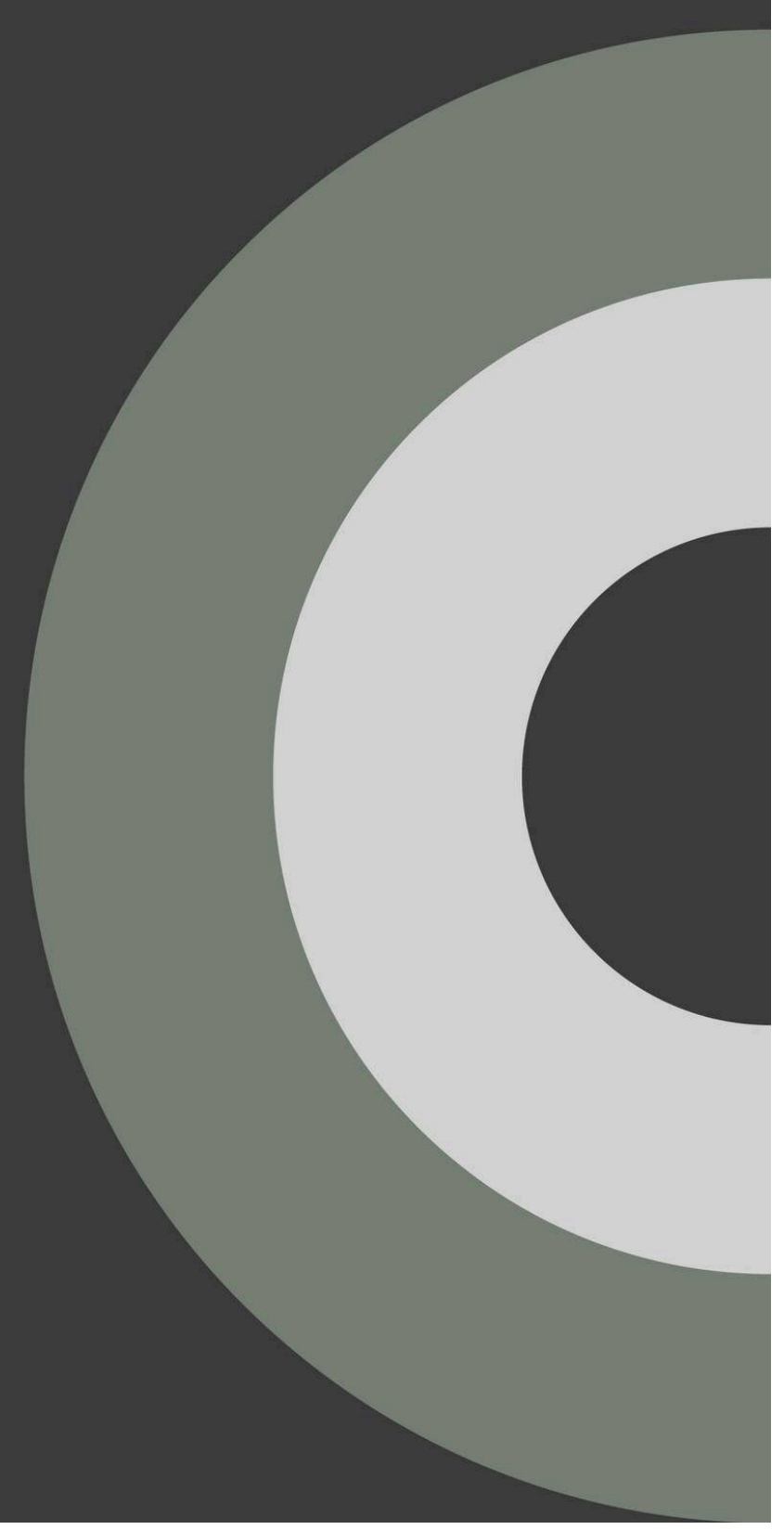




\section{La transmission des savoirs à l'ère numérique}

\section{Arnaud Dhermy (dir.)}

Éditeur : Éditions du Comité des travaux historiques et scientifiques Année d'édition : 2021

Date de mise en ligne : 8 juin 2021

Collection : Actes des congrès nationaux des sociétés historiques et scientifiques

EAN électronique : 9782735509027

\section{Sbooks}

http://books.openedition.org

\section{Référence électronique}

DHERMY, Arnaud (dir.). La transmission des savoirs à l'ère numérique. Nouvelle édition [en ligne]. Paris : Éditions du Comité des travaux historiques et scientifiques, 2021 (généré le 10 juin 2021). Disponible sur Internet : <http://books.openedition.org/cths/14988>. ISBN : 9782735509027.

Ce document a été généré automatiquement le 10 juin 2021.

(C) Éditions du Comité des travaux historiques et scientifiques, 2021

Conditions d'utilisation :

http://www.openedition.org/6540 
Nouveaux outils, nouveaux usages, nouveaux publics : la révolution technologique a changé la manière de produire, organiser et partager les savoirs. Pour interroger les modalités de cette transmission, l'ouvrage donne la parole à des chercheurs et à des collectionneurs. La diversité de leurs témoignages offre un ensemble original de réflexions et d'orientations méthodologiques qui sont autant d'études de cas dans un contexte de mobilités ergonomiques et participatives.

Le Congrès national des sociétés historiques et scientifiques rassemble chaque année universitaires, membres de sociétés savantes et jeunes chercheurs. Ce recueil est issu de travaux présentés lors $\mathrm{du} 143^{\mathrm{e}}$ Congrès sur le thème « La transmission des savoirs ».

\section{ARNAUD DHERMY}

Docteur en histoire des mondes modernes et contemporains

Chef de la mission de la Coopération régionale et responsable du programme de numérisation des publications des sociétés savantes à la Bibliothèque nationale de France 
SOMMAIRE

Introduction

Arnaud Dhermy

Faire et transmettre l'histoire de la justice : les enjeux du numérique

Marc Renneville, Jean-Lucien Sanchez et Sophie Victorien

Criminocorpus : une plate-forme numérique pour l'histoire publique de la justice

Transmettre l'histoire par le numérique

Transmission numérique des savoirs : quel type de vidéos, quel rôle des structures publiques?

Alexandre Moatti

Système de formation en ligne et norme de mérite : le projet TeSLA

Nathan De Vos

Méthodologie de l'enquête

Qu'est-ce que TeSLA?

Processus d'appropriation par les utilisateurs

Un mérite individualisé ?

Les enjeux de la numérisation des cartes et photographies anciennes dans la transmission des savoirs

Daniel Pouyllau et François Bart

Les fonds de cartes et de photographies anciennes à Bordeaux

Techniques et méthodes utilisées pour la numérisation et la description des cartes et des

photographies

La diffusion des cartes et photographies et la transmission des savoirs

Des catéchismes à Voltaire : le projet MEDIATE et le rôle des livres « moyens » dans la transmission culturelle à l'époque des Lumières

Alicia C. Montoya

Étudier le champ littéraire du XVIII ${ }^{\mathrm{e}}$ siècle

À la recherche des auteurs middlebrow

Définir la littérature de culture moyenne

Un phénomène éditorial européen

Des livres religieux, des livres conservateurs?

La base de données et le projet numérique MEDIATE

Le monument pour transmettre, la carte postale pour diffuser : de la collection à la base de données

France Debuisson-Lagneau

Cartes postales et illustres

La carte postale comme recours aux monuments défaillants

Vers la base de données

Un plaidoyer pour la transmission des bibliothèques privées des intellectuels et enseignants :

la bonne fortune du numérique

Odile Jacquemin

L'origine du propos

Un panel de douze bibliothèques pour illustrer la diversité des situations

Questionnements et pistes 
NOTE DE L'ÉDITEUR

Les articles de cet ouvrage ont été validés par le comité de lecture des Éditions du Comité des travaux historiques et scientifiques dans le cadre de la publication des actes du $143^{e}$ Congrès national des sociétés historiques et scientifiques tenu à Paris en 2018. 


\title{
Introduction
}

\author{
Arnaud Dhermy
}

1 Parmi les nombreuses contributions du $143^{\mathrm{e}}$ Congrès national des sociétés historiques et scientifiques consacrées à la transmission des savoirs, celles abordant le tournant numérique n'avaient pas fait l'objet d'une séance spécifique. Évoquée au gré des sessions, cette problématique a cependant constitué comme une trame de fond de plusieurs interrogations qui justifiait amplement la constitution du présent volume.

2 L'ère numérique accentue certes une diversification des savoirs et pose le défi d'une dispersion documentaire. Toutefois, les contributions ici rassemblées s'inscrivent moins dans une réflexion sur cette massification que dans une interrogation sur la manière de partager ce savoir en ligne. De même, elles questionnent les polarités entre lesquelles s'opèrent désormais cette transmission et plus largement l'adaptabilité de sa médiation.

3 De fait, parce que l'accès en ligne le permet, la transmission des savoirs à l'ère numérique implique le principe d'une mise à disposition universelle des contenus. Il s'agit désormais d'appréhender l'irrigation de communautés virtuelles dont ni les contours ni la nature n'ont a priori pu être exclusivement définis. Cette situation ne concerne pas seulement le partage des connaissances mais aussi leur organisation; en découle alors, en fonction des publics concernés, une interrogation qui s'élargit aux modes d'enrichissement de ces savoirs eux-mêmes.

4 Les contributions qui suivent se réfèrent d'une manière ou d'une autre à ces problématiques. Certaines expériences relatées abordent la porosité des périmètres de connaissance qu'entraîne une mise en réseau; d'autres reviennent sur l'opportunité que constitue une interconnexion entre communautés de recherche; d'autres en revanche font le constat d'une inadéquation persistante des outils de médiation disponibles, ou des intuitions ergonomiques qui les ont générés, en rapport à la diversité des profils ou aux usages auxquels ils ont finalement été confrontés. Toutes cependant retiennent le postulat d'une forme de public de plus en plus impliquée, non seulement en tant que consommateur de savoirs, mais aussi de plus en plus en tant que participant, dans un processus élargi de dynamiques documentaires, voire même d'expertises. 
5 La réflexion sur la transmission des savoirs à l'ère numérique, à lire ce qui suit, se poserait donc davantage à partir de l'émulation proactive qu'offre désormais toute mise en ligne. De même, elle ne pourrait se dissocier des problématiques se rapportant à la production de connaissances à laquelle participent de façon délibérée des acteurs non scientifiques - ce qu'on qualifierait volontiers aujourd'hui de production participative -, voire même elle ne pourrait se dissocier des problématiques sur l'élaboration de connaissances non plus destinées à acquérir un statut académique mais à constituer de "nouveaux savoirs", par exemple en matière de gestion de flux d'informations, en matière d'innovation, de pratiques interdisciplinaires et collaboratives.

6 Ce questionnement débouche sur celui des interfaces entre périmètres de recherche ou entre communautés de recherche, de même que sur celui du statut de transmetteurs et d'experts. Cette réflexion doit aussi être considérée en lien avec la constitution coopérative et comme au fil de l'eau des corpus qui densifie d'autant les ramifications et les segmentations des savoirs, qui complexifie les échanges au sein des réseaux de recherche et des milieux de la transmission.

7 Cette réflexion concerne également les supports de ces savoirs, leur ergonomie et plus largement les capacités de partage qu'offre l'ère numérique: la compatibilité universelle des espaces d'échanges et de transmission notamment. Elle touche enfin aux processus de qualification, de validation scientifique, y compris d'éléments de connaissances extra-académiques, par exemple ceux destinés à alimenter les débats sociétaux, politiques, ou à donner de la profondeur aux commémorations ou aux célébrations populaires.

8 Dans ce contexte se renforce la formation de certains pôles de connaissances constitués d'émetteurs de sources identifiés à la fois comme inventeurs et consommateurs. Décrire le réseau de telles transmissions des savoirs confinerait paradoxalement à désigner une forme plus traditionnelle de parité émulative, une forme renouvelée et étonnamment pérenne de sociabilité savante caractérisée par le truchement d'apprentissages indirects, croisés, alternatifs, voire informels, plutôt qu'à travers un apprentissage essentiellement descendant, qualifiant ou institutionnel.

C'est finalement sur cette interrogation partagée que réside le fil conducteur qui pourrait le mieux qualifier ce volume: celle découlant du constat d'un dérèglement croissant et inéluctable dans le rapport traditionnel entre transmetteur et récepteur d'un savoir, une fois celui-ci parvenu à l'ère numérique. D'où le besoin évoqué dans certaines contributions qui vont suivre d'une interrogation plus poussée sur les polarités de cette transmission. Au-delà du thème d'un désenclavement des ressources disponibles, il sera donc question des modalités de partage, puisque la manipulation d'un matériau mis en réseau ne saurait plus dépendre d'une communauté homogène, parce qu'elle ne saurait plus se cantonner à une même ergonomie ni à des outils d'exploitation exclusifs.

10 Naturellement, les contributions rassemblées ici n'offrent pas un panorama complet de toutes ces réflexions. Positionnées à différents stades des problématiques qui viennent d'être esquissées, elles forment même, il faut le reconnaître, un ensemble assez hétérogène qui ne saurait retranscrire une démarche linéaire. De même, elles 
constituent moins une suite de bilans ou de synthèses qu'une présentation de projets ou d'intuitions. Mais, dans le contexte qui vient d'être exposé, chaque sujet constitue malgré sa spécificité une suggestive étude de cas.

11 Ce volume laisse aussi pendantes, bien évidemment, plusieurs questions: celle notamment d'une disparité des savoirs qu'engendre un transfert progressif mais discontinu d'objets sur support numérique, celle aussi d'un manque de structuration globale, questions qui n'apparaissent qu'en filigrane dans les exposés réunis ici. Ces textes doivent donc être lus pour ce qu'ils sont : non pas un état complet de la question mais les pièces d'une réflexion en devenir dont on ambitionne de poser ici quelques points d'étape, d'une réflexion autour d'un contexte de mobilités méthodologique et ergonomique découlant de modes désormais virtuels de diffusion et d'espaces d'échange.

12 À travers une expérience pionnière de partage de savoirs telle qu'il pouvait en être envisagé dès 2003 - celle de Crimonocorpus -, Marc Renneville ouvre ici fort opportunément le recueil. Le retour qui est fait sur ce chantier né d'une volonté de diffuser un savoir centré sur l'histoire de la justice, des crimes et des peines rappelle l'intuition première de cette plate-forme de ressources - dont quelques-unes déjà étaient nativement numériques - incluant des outils, des informations de référence destinés à une communauté scientifique, mais aussi des savoirs susceptibles de nourrir une appropriation critique de la part d'un lectorat peu familier des sciences juridiques. Cette expérience a visé autant une démarche muséale et éditoriale innovante qu'une offre ouverte de réappropriation, de par la diversité des accès. Dans le cadre de la constitution d'un savoir numérique et de ses différentes ramifications, la construction de Criminocorpus est intéressante par ses porosités internes et ses possibilités de rebonds ; expérience qui se distingue donc moins ici en matière de structuration que de mise en réseau de ses composants.

13 Marc Renneville pose ainsi l'amorce d'une réflexion présente dans l'ensemble du présent volume: celle qui concerne une production de contenus «qu'il n'est plus possible d'organiser dans une forme narrative linéaire et univoque ", placée au centre d'une variété d'outils susceptibles de nourrir l'appropriation individuelle. En même temps, Criminocorpus est l'opportunité d'une mise en place des conditions d'un travail collectif, notamment incluant des spécialistes issus d'autres disciplines voire d'interlocuteurs qui ne relèvent pas du monde de la recherche mais qui disposent de connaissances complémentaires qu'il s'agit de combiner au sein d'environnements intégrés, dépendants de fonctionnalités spécifiques: un site web indépendant, un musée numérique, un carnet de recherche, une base de données.

Dans le prolongement de Criminocorpus, les trois contributions suivantes évoquent les problématiques de la substitution numérique à une interaction traditionnelle de savoirs, dans le dessein d'une mise à la disposition des publics à la fois plus pratique et plus universelle. Elles prolongent notamment la réflexion sur les remises à plat nécessaires que génèrent - dans la mesure où ce sont bien les contenus qui conditionnent une réflexion ergonomique et non l'inverse - la volonté d'un partage fluide de contenus et l'interaction que cela entraîne entre des communautés ou des dispositifs de statuts différents. 
15 En premier lieu, Alexandre Moatti aborde la question délicate de la continuité entre pédagogie scientifique hors les murs et canaux de diffusion, notamment par les réseaux sociaux, dans un contexte de densification où la faveur des publics ne se porte pas tant sur le média le plus approprié que sur sa popularité ou son accessibilité. Alexandre Moatti s'interroge à juste titre sur le rôle que devraient continuer d'occuper sur ce point les institutions publiques, non seulement pour assumer et défendre en ligne une médiation de qualité, voire qualifiante, mais aussi, à l'instar d'autres structures documentaires nationales, pour garantir une authentification, une labellisation des savoirs du milieu des contenus de toutes sortes mis à la disposition du grand public.

C'est sur une problématique proche, celle de la certification académique, que Nathan De Vos aborde à son tour les formes de substitution numérique de la transmission des savoirs, à travers un projet de formation en ligne intitulé TeSLA et la question de l'authentification à distance des candidats dans le cadre d'examens qualifiants, en lieu et place d'interactions en présence traditionnelles. À cet égard, il pose dans ce volume une problématique que l'on retrouvera plus loin : celle des ergonomies expérimentales confrontées à l'appropriation des publics.

17 Autre retour d'expérience sur les enjeux de mises à la disposition des publics et d'accessibilité, celui proposé par Daniel Pouyllau et François Bart en rapport cette fois avec les ressources documentaires que sont les matériaux cartographiques et photographiques. Ce projet de diffusion en ligne émane du centre d'information scientifique et technique du CNRS Regards et du consortium «ImaGEO, Images des géographes - Cartes et photographies ", combinés au sein de l'université BordeauxMontaigne. Sujet somme toute classique, la valorisation des collections numériques propre aux services documentaires des bibliothèques ou centres d'archives s'ouvre ici à certaines actions participatives visant à renseigner et indexer de manière plus appropriée les ressources du patrimoine scientifique, et à leur permettre une mise en miroir avec les problématiques et les matériaux du temps présent.

Déjà évoquées ici à propos du projet Criminocorpus, les bases de données considérées comme vectrices du traitement de matériaux de recherche et contribuant de ce fait à constituer des ensembles documentaires sont à nouveau abordées à travers la présentation par Alicia C. Montoya du projet MEDIATE. Cette entreprise basée à l'université Radboud de Nimègue (Pays-Bas) est consacrée à la réinvention d'un corpus documentaire à partir de l'analyse des volumes de diffusion d'une certaine littérature aujourd'hui tout à fait oubliée mais qui connut au xviII ${ }^{\mathrm{e}}$ siècle un grand succès éditorial et commercial.

Il y avait nécessité d'effectuer une approche quantitative - les catalogues de vente de bibliothèques - seule à même de digérer des centaines de milliers de références. Outre des rapprochements avec d'autres problématiques de recherche, le traitement numérique des données ouvre la voie à d'autres méthodologies, par exemple en incluant l'emploi d'instruments d'analyse utilisés plus couramment pour décrire les réseaux sociaux. Ces rapprochements permettent d'obtenir des résultats inédits portant sur des sources demeurées jusque-là enclavées, dispersées. Le projet MEDIATE illustre bien l'opportunité que représente un traitement numérique des matériaux pour une ouverture des périmètres de recherches et d'échanges. Le modèle de la base de 
données présentée ici, enrichie au fil de l'eau, permet en outre d'envisager à terme l'invention d'autres corpus et la constitution de nouveaux écosystèmes, dans la mesure où une véritable «mise en gamme » du savoir disponible devient possible, offrant en puissance d'autres modalités de transmission.

La réflexion qui fait suite aborde moins quant à elle l'invention de périmètres d'étude que les contraintes propres à la compilation des matériaux utiles à la recherche au sein d'un corpus déjà délimité. Cette problématique est à la base d'une convergence vertueuse que relate France Debuisson entre plusieurs catégories d'acteurs (collectionneurs amateurs, conservateurs, historiens de l'art et universitaires) autour d'un matériau populaire mais encore peu structuré, celui des cartes postales anciennes, notamment inspirées de la statuomanie qui a existé sous la III République. La diversité des approches et des objectifs réunis dans ce contexte suscite aussi un défi : celui de la manipulation fédérée des sources. Le projet de base de données de cartes postales À nos grands hommes témoigne de la recherche d'un processus qui, à l'écart d'une démarche qui se caractériserait par une constitution centralisée des connaissances, naît au contraire de chaque granularité d'appréhension des sources: depuis l'étape du collectage jusqu'à celle de l'analyse mais aussi, selon les communautés de chercheurs qui s'y emploient, de chaque niveau envisagé de mise à la disposition des publics.

21 La base de données À nos grands hommes, qui est née de cette convergence, illustre par ailleurs un type de projet réalisé sur la base de partenariats extra-institutionnels répartis non plus entre différentes spécialités, mais dans le cadre d'un processus concerté d'invention: signe que la constitution d'un périmètre de recherche sous forme numérique est susceptible de bousculer une articulation traditionnelle dans le traitement des données, et qu'à la constitution fédérée de nouveaux ensembles documentaires s'ajoute désormais une diversité et une répartition des axes d'appropriation comme des analyses par-delà les institutions et espaces de recherche.

Le volume s'achève sur une perspective originale : l'appel lancé par Odile Jacquemin pour une prise en considération de la documentation personnelle des intellectuels matériau lui-même constitué au fil de l'eau, volatil - et en faveur de sa pérennisation grâce à «la bonne fortune du numérique ». Est notamment esquissée ici la question d'une nouvelle strate de savoir, par la captation d'une étape du processus scientifique à travers sa seule matérialisation numérique: un essai d'instantané pour ainsi dire, réalisé à partir des réflexions esquissées par les chercheurs, qu'il s'agit de considérer comme matériaux en soi, distincts du résultat de recherche lui-même et notamment de sa manifestation textuelle. Une telle médiation numérique permet d'isoler un auteur à partir de ses notes, de son histoire et de sa documentation personnelle, rendues exploitables sous la forme d'apostille numérique à une œuvre par ailleurs publiée, démarche de cristallisation à laquelle l'auteur lui-même peut être invité à s'associer.

Par-delà les contraintes évidentes que supposeraient la patrimonialisation physique de l'intégralité d'une bibliothèque ou encore la restitution de sa logique de classement et d'accroissement, il s'agit d'un pari lancé pour une meilleure prise en compte des sécrétions méthodologiques et analytiques attachées à la recherche: annotations marginales et billets griffonnés encore en place dans les ouvrages, mais aussi échanges informels... toutes sortes d'à-côtés remettant, précise Odile Jacquemin, "parole et langage au cœur des projets, dans un échange ouvrant le passage de l'écrit à l'oral » étroitement associés et dépendants à la fois du témoignage vivant de l'auteur et de la médiation pérenne qu'il faudra lui attribuer pour lui conserver sa pertinence, en marge 
d'autres formes que sont les expositions virtuelles, les scénographies, les archives sonores, les entretiens ou les discussions retranscrites. Elle se place en tout cas sous un angle différent que ce que l'on pourrait par exemple exploiter en matière de paratexte, pour assumer un autre mode de transmission y compris muséale, dans un contexte cognitif virtuel anticipant l'inéluctable dispersion physique de ces cabinets de connaissances.

La transmission des savoirs à l'ère numérique entrâne indéniablement une déstabilisation des pratiques, des savoir-faire, à la fois du médiateur et du chercheur mais aussi dans les interactions qui leur sont propres. De fait, la légitimité d'un savoir à transmettre, en paraphrasant Antoine Prost, n'est pas la propriété de spécialistes dont la pertinence sur un sujet donné viendrait d'une "supposée maitrise d'une méthode inaccessible au profane; elle tient [en revanche] au travail qu'ils ont accumulé, aux connaissances qu'ils ont lentement amassées. Cela leur permet de valider certaines affirmations, certains choix ${ }^{1} »$. C'est pourquoi, au moment où la transmission de l'information est confrontée au défi de l'abondance, la conception traditionnelle d'une répartition vertueuse entre transmission et réception ne tient plus. Quelles seraient par exemple les conséquences d'un repli assumé par certaines communautés de recherche face à cette profusion, ou même le choix de leur part d'une utilisation sélective ? Se présente en revanche une réelle opportunité en faveur d'une extension des compétences attribuables aux différents acteurs potentiels de la production et de la transmission des savoirs, une opportunité en faveur d'une réactivation des parités entre démarches savantes de tout statut, académiques et extra-académiques, en vue d'une investigation répartie des nouveaux gisements de données. Toutefois, comme le rappelle Marc Renneville dans l'intervention qui va suivre, il convient de veiller «à ce que cette opportunité ne soit pas gâchée au profit des mirages du technologisme ».

\section{NOTES}

1. Antoine Prost, "Transmettre l'histoire", dans Chemins d'accès : quelle(s) médiation(s) pour l'histoire? ge rencontres des services éducatifs de musées, bibliothèques, archives et théâtres, éd. numérique, Paris, BNF, 2011, p. 7. [URL: http://classes.bnf.fr/actes/9/ Prost.pdf] 


\section{AUTEUR}

\section{ARNAUD DHERMY}

Docteur en histoire des mondes modernes et contemporains

Chef de la mission de la Coopération régionale et responsable du programme de numérisation des publications des sociétés savantes à la Bibliothèque nationale de France 


\title{
Faire et transmettre l'histoire de la justice : les enjeux du numérique
}

\author{
Marc Renneville, Jean-Lucien Sanchez et Sophie Victorien
}

\begin{abstract}
« La connaissance historique est, comme la connaissance d'autrui, comme la connaissance de soi, un cas particulier de la connaissance de

l'homme, et participe à son incertitude, à sa liberté essentielle. » (H.-I. Marrou, « Tristesse de
\end{abstract}

l'historien », p. 116.)

1 Conçu en 2003 par des historiens associés à des archivistes et des documentalistes, le projet Criminocorpus est né d'une intention historiographique et d'une volonté de diffusion du savoir historique en libre accès qui a marqué le paysage numérique français par sa logique thématique centrée sur l'histoire de la justice, des crimes et des peines. Le néologisme Criminocorpus a été forgé pour désigner initialement un site web qui s'est enrichi de nouvelles fonctionnalités, d'un blog externe et d'une revue. En 2016, le site web principal a été restructuré pour devenir le premier musée numérique d'Histoire de la justice, des crimes et des peines. Nous proposons de retracer ici notre démarche de transmission auprès de plusieurs lectorats identifiés (historiens, professionnels de la justice, étudiants...) et au-delà, de toute personne intéressée, en la situant dans l'historiographie de notre discipline. Nous expliciterons notre projet de promouvoir une histoire de la justice au sens large en exposant ses motivations, les aléas de nos réalisations mais également les conséquences sur notre métier d'historien de cette démarche de passeurs de savoirs et de savoir-faire.

\section{Criminocorpus : une plate-forme numérique pour I'histoire publique de la justice}

2 Criminocorpus est une plate-forme numérique francophone et partiellement plurilingue gratuite en ligne (fig. 1). Elle couvre tous les aspects de la recherche en histoire de la justice, des crimes et des peines. Le site Criminocorpus a été ouvert en 2005 à la suite 
d'une action concertée incitative du CNRS, «Histoire des savoirs ", dirigée par Marc Renneville, ce qui en fait un site relativement ancien dans le domaine des humanités numériques. Les contenus de Criminocorpus sont édités par le Centre pour les humanités numériques et l'histoire de la justice (CLAMOR) depuis septembre 2015. Il s'agit d'une unité mixte de service créée par le CNRS et le ministère de la Justice en partenariat avec les Archives nationales (UMS CNRS 3726). Assurant la maintenance et l'évolution de Criminocorpus, le CLAMOR porte une offre de services auprès de la communauté scientifique. Cette offre consiste à concevoir, éditorialiser ou rendre disponibles en libre accès sur le web des instruments de recherche, des corpus documentaires et des publications de formes très variées (article, exposition, synthèse, note documentaire, archive commentée...). Le CLAMOR développe son offre éditoriale sur la base d'une infrastructure numérique opérée par la très grande infrastructure de recherche HumaNum ${ }^{1}$ dédiée aux humanités numériques. Cet hébergement permet une mise en ligne pérenne et stable des données.

Fig. 1. - Criminocorpus, page d'accueil.

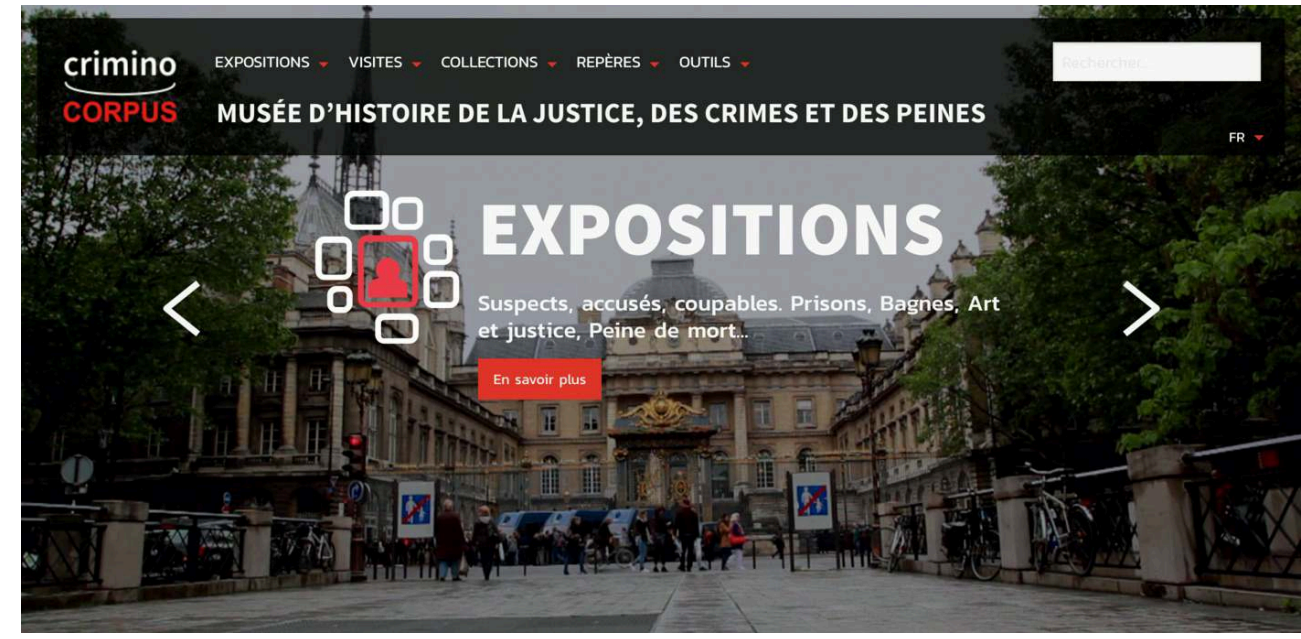

Capture d'écran (2018).

(c) Criminocorpus.

3 La plate-forme Criminocorpus a considérablement évolué tant sur le plan technologique que dans sa structuration. Le site portail initial est devenu depuis 2016 un musée dédié à l'histoire de la justice, le premier nativement numérique. Tous les contenus en ligne sur Criminocorpus sont accessibles gratuitement et la plate-forme se décline sous la forme de trois sites :

- un musée d'Histoire de la justice ${ }^{2}$;

- une revue scientifique ${ }^{3}$;

- et un carnet de recherche ${ }^{4}$.

4 L'objectif de transmission de savoir porté par Criminocorpus n'est pas de produire une histoire visant le grand public. L'exigence de notre démarche consiste à produire une offre de contenus, destinée à un public diversifié, composé de lectorats identifiés dont les attentes peuvent se recouper ou diverger.

Les thématiques de notre champ d'étude étant une ressource pour le débat public, les dossiers et les outils que nous proposons s'adressent simultanément à la communauté scientifique et à tout lecteur intéressé, les contributions publiées conciliant valeur 
scientifique, accessibilité et clarté du propos. Toutefois, bien que «la fonction sociale de l'histoire oblige [...] les historiens à prendre en charge la mémoire collective du pays ${ }^{5}$ ", nous n'entendons pas établir notre politique de publication en réponse à la demande sociale de mémoire ou à des prises de position militantes. Déjà, en 1982-1983, le séminaire de François Bédarida «L'histoire du temps présent et ses usages: recherche fondamentale et histoire appliquée » abordait cette question de la demande sociale, sa nécessaire mise à distance et le rôle de l'histoire et des historiens dans la

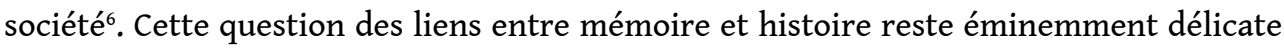
comme en témoigne l'action du Comité de vigilance face aux usages publics de l'histoire fondé à la suite de la loi du 23 février 2005 « exigeant des enseignants qu'ils insistent sur "le rôle positif" de la colonisation ${ }^{7}$ » ou de "Liberté pour l'histoire » née la même année en opposition aux lois mémorielles ${ }^{8}$. La dimension d'« histoire publique» inhérente au projet Criminocorpus consiste à rendre publiques et accessibles des ressources et elle est donc bien différente, en ce sens, de la public history américaine. Elle n'indique pas notre appartenance à un mouvement politique ou un quelconque activisme mais la volonté de toucher un public au-delà de la communauté scientifique.

Mais si notre projet éditorial n'entend pas répondre à la demande sociale de mémoire, que peut-il apporter à ses contemporains ? Doit-il tendre - par souci d'exhaustivité impartiale - à la restitution d'un passé totalisant tous les faits advenus, sans en négliger aucun?

« L'histoire, au contraire, écrit Lucien Febvre ${ }^{9}$ dans un texte célèbre, est appel à "ne pas se laisser écraser par cette accumulation inhumaine de faits hérités. Par cette pression irrésistible des morts écrasant les vivants - laminant sous leur poids la mince couche du présent jusqu'à lui enlever toute force de résistance". C'est "un moyen d'organiser le passé pour l'empêcher de trop peser sur les épaules des hommes". Elle interroge la mort en fonction de la vie. Dans ces réorganisations successives, certains faits passent au second plan, certains événements sont écartés : l'histoire aménage l'oubli, sélectif mais inéluctable. L'histoire, elle, est du côté de la connaissance, du savoir ; elle est mise à distance, rationalisation, volonté de comprendre et d'expliquer ${ }^{10}$."

Il nous semble ainsi que nous faisons moins œuvre de simplification et de réduction de l'histoire de la justice qu'une présentation de la recherche en train de se faire à travers non seulement des articles, mais aussi toute une palette d'outils en offrant différents niveaux d'accès à la connaissance de l'histoire de la justice grâce aux travaux de chercheurs et de sources variées. Henri-Irénée Marrou soulignait que :

«[...] si l'histoire se montre si "nourrissante" cela tient aussi à ce qu'elle ne nous met pas en présence d'un donné humain brut, mais que l'historien, et avant lui ses sources, ont déjà élaboré, rendu assimilable, compréhensible, rationnel ${ }^{11}$ ».

L'histoire de la justice étant un champ de connaissance sensible susceptible de provoquer des interprétations contradictoires, voire conflictuelles, Criminocorpus n'accueille aucune parole ou position militante contemporaine. La connaissance historique que nous entendons promouvoir n'a pas la prétention de donner, à elle seule, des leçons aux politiques présentes. Elle vise à mettre à la libre disposition des visiteurs des informations fiables et des outils pour nourrir une appropriation critique des représentations partagées sur le temps présent :

«Les savoirs ne sont pas comme des cailloux que le professeur puiserait dans sa musette et donnerait aux élèves pour qu'ils les mettent dans la leur. Savoir, c'est être capable de produire un discours qui donne du sens aux objets et aux dates ${ }^{12}$. » 


\section{Transmettre l'histoire par le numérique}

9 Si la rédaction d'un article ou d'un ouvrage correspond à une forme de diffusion traditionnelle des connaissances bien maîtrisée par les historiens, l'informatique et le numérique ont ouvert la voie à la production de contenus hypertexte et hypermédia qu'il n'est plus possible d'organiser dans une forme narrative linéaire et univoque. Publier en ligne n'est pas pour l'équipe de Criminocorpus un choix par défaut mais un moyen d'expérimenter de nouvelles écritures et des modes de restitution inédits du savoir historique. Le libre accès à des savoirs spécialisés génère en effet des opportunités et des contraintes dont il convient de tenir compte. Une publication scientifique en ligne présente de fortes chances d'être lue par un public non spécialiste et les textes et documents peuvent être reliés à d'autres textes et documents en ligne, ce qui rend possibles les lectures buissonnières, les rebonds d'une source à une autre. Toutefois, le risque est grand, si ce cheminement n'est pas guidé, de contribuer involontairement à une appréhension dispersée des connaissances. Notre conviction est que l'historien ne peut y parer seul. Il doit impérativement agir collectivement en faisant appel à des compétences tierces s'il veut construire un espace de savoir alliant qualité et accessibilité.

10 Cette exigence est probablement inhérente à tout projet de transmission de connaissance mais les écritures numériques n'en sont aujourd'hui encore qu'au stade de l'expérimentation. La conséquence directe des potentialités de l'hyperlien, c'est que l'éditorialisation en ligne ne peut se réduire à déposer un texte dans un espace de lecture à distance. Il faut imaginer, tester et réajuster sans cesse. L'organisation de la plate-forme Criminocorpus n'a cessé ainsi d'évoluer au fil de l'enrichissement de son contenu et de l'évolution des technologies disponibles ${ }^{13}$. La description que nous proposons ci-après de notre musée est donc, à n'en pas douter, tout à la fois actuelle et datée.

11 Le musée d'Histoire de la justice de Criminocorpus propose cinq espaces: des expositions, des visites pouvant toucher un large public, une bibliothèque numérique, des outils et des repères destinés a priori à un public plus spécialisé. Ces rubriques ont été pensées notamment en fonction des profils des utilisateurs potentiels. Or les premiers résultats de deux études d'ergonomie cognitive menées sur la fréquentation de notre site révèlent que la perception de notre musée est différente de celle que nous avions imaginée. Si plusieurs publics se rendent effectivement sur le site, ils ne perçoivent pas forcément l'organisation des ressources de la même manière. La leçon que nous tirons de ce constat est simple et nous renvoie à la didactique des disciplines : le savoir de l'historien n'est pas suffisant pour trouver les bonnes stratégies d'appropriation des contenus disponibles. S'agissant de publication numérique, l'apport de l'ergonomie cognitive sera sollicité pour concevoir l'évolution de nos futures interfaces ${ }^{14}$.

\section{Les expositions}

12 Le musée donne accès actuellement à trente-neuf expositions virtuelles qui se répartissent à travers cinq parcours : "En prison », "Au bagne ", "Suspects, accusés, coupables », « Art et justice » et « Peine de mort ». Ces expositions sont des productions numériques originales ou le portage en ligne d'expositions initialement organisées dans 
des musées, des bibliothèques ou des centres d'archives. Ce portage permet ainsi aux auteurs de voir leur travail valorisé et conservé sur le long terme. Mais cela permet surtout, dans le cadre du portage d'une exposition institutionnelle, de l'ouvrir à un plus grand nombre de visiteurs en élargissant considérablement le spectre de sa diffusion. Citons par exemple le partenariat établi entre Criminocorpus et les Archives nationales qui a permis la mise en ligne de l'exposition « Fichés ? Photographie et identification du Second Empire aux années $60 \%$. Alors que cette exposition n'était visible que du 27 septembre au 26 décembre 2011 aux Archives nationales (site de l'hôtel de Soubise), elle est désormais accessible en ligne (dans sa déclinaison numérique), et ce sans limite de temps.

Ces expositions peuvent être soit uniques (mais leurs auteurs conservent la possibilité de les modifier a posteriori), soit ouvertes. Dans ce cas, il s'agit d'expositions qui peuvent être enrichies au fil de l'eau, par exemple l'exposition "La mémoire des murs", coordonnée par Jean-Claude Vimont. Cette exposition, dédiée aux graffitis pénitentiaires, se décline sous la forme de chapitres réalisés par différents auteurs: "Les graffitis de la maison centrale de Clairvaux» par Laure Bulmé ; «Les graffitis contemporains du château de Gaillon » par Dominique Pitte, France Poulain et JeanLouis Breton, etc.

14 Certains auteurs sollicitent spontanément Criminocorpus tandis qu'une veille assurée par l'équipe permet également de démarcher directement des auteurs. Le portage en ligne est assuré gratuitement par nos soins et l'auteur reste maître d'œuvre jusqu'à la publication finale: le projet de numérisation est discuté et élaboré en amont avec l'équipe de Criminocorpus qui accompagne l'auteur tout au long du processus de mise en ligne. En parallèle, certaines expositions virtuelles sont traduites en anglais afin de les rendre accessibles au public anglo-saxon (et au-delà). Ces expositions virtuelles constituent une porte d'entrée extrêmement attractive pour capter et intéresser un public large et pas forcément sensibilisé à l'histoire de la justice. Elles constituent également un module qui permet de vulgariser et de rendre accessibles auprès du plus grand nombre des productions scientifiques relativement exigeantes. Elles permettent ainsi aux historiens de diffuser leurs travaux de recherche auprès d'un public auquel ils n'étaient pas forcément destinés initialement.

\section{Les visites}

15 Les visites virtuelles permettent au public de pénétrer et de découvrir des lieux de justice habituellement fermés, réservés ou disparus comme la prison de la Santé à Paris, l'ancienne prison du Havre et, depuis le mois d'octobre 2016, le palais de justice de Paris. Ces parcours multimédia donnent accès au fil du thème choisi à la visite des lieux, à des entretiens filmés, des témoignages, des objets du quotidien et des documents rares. Chacune de ces visites est l'occasion de suivre des liens vers les autres ressources du musée et de la revue Criminocorpus. Ces visites œuvrent à la conservation patrimoniale des lieux de justice en permettant, pour ceux qui ne sont désormais plus visibles ou qui ont subi d'importants travaux à la suite de leur fermeture, de pouvoir en conserver la trace. Ce portage en ligne permet ainsi de saisir à un moment donné le fonctionnement et l'état d'un bâtiment puisque chacune de ces visites est présentée par des agents y ayant exercé tout ou partie de leur carrière. Par exemple, pour la maison d'arrêt de la Santé, fermée en juillet 2014 pour la réalisation d'une importante 
campagne de travaux, un surveillant présente chacune des pièces accessibles en prenant le soin de fournir des informations aux visiteurs. Ces visites permettent ainsi de conserver le témoignage des pratiques professionnelles de ces agents. Et cela permet en parallèle aux agents de pouvoir conserver la mémoire de ces lieux. Elles disposent également d'une dimension civique en permettant à tout citoyen de pénétrer dans des lieux de justice, notamment des lieux d'exécution des peines comme les prisons, et de prendre conscience de la réalité de la détention et de l'organisation de la justice.

\section{La bibliothèque}

La bibliothèque donne accès à des collections thématiques du musée qui rassemblent un ensemble documentaire particulièrement riche sur l'histoire de la justice, des crimes et des peines. Les documents numérisés sont des reproductions dont les originaux sont conservés dans différents services, institutions publiques (Archives nationales, archives départementales, musées, bibliothèques...), ou qui appartiennent à des collections privées (chercheurs, collectionneurs...). Les collections donnent ainsi accès à des documents de natures très diverses: manuscrits, tapuscrits, imprimés, sources sonores ou vidéos, plaques de verre, articles de journaux, ouvrages rares, photographies... On trouve notamment deux séries de journaux de faits divers criminels de l'entre-deux-guerres conservés par la Bibliothèque des littératures policières : une série complète de Police magazine couvrant la période 1931-1939 (plus de 350 numéros) et une série complète de Détective couvrant les années 1928 à 1940 (582 numéros). Ces séries ont fait en outre l'objet d'un traitement par reconnaissance optique de caractères (OCR) qui permet aux lecteurs de les interroger par mots-clés, facilitant ainsi la navigation et l'utilisation de ces données. Les collectionneurs qui confient leurs images conservent la possibilité d'enrichir leur fonds au fur et à mesure.

\section{Les repères}

Le musée offre également différents « Repères » qui mettent à la disposition du public des corpus juridiques, des chronologies et les textes de loi qui y sont liés. On trouve notamment toutes les versions du Code civil, de 1804 à 2004, ainsi que toutes les versions de l'ordonnance du 2 février 1945 sur la justice des mineurs. Enfin deux nouveaux modules en ligne se détachent des autres repères par leur caractère participatif mais aussi car ils touchent un public beaucoup plus large. HUGO, dédié à la connaissance du patrimoine judiciaire, vise à dresser un inventaire patrimonial des lieux de jugement et d'exécution des peines en rassemblant des informations relatives à l'architecture, l'histoire et la mémoire des lieux. HUGO propose une interface de consultation bilingue en libre accès destinée à faciliter les recherches sur le patrimoine judiciaire. Conçu sur une base participative, il offre à tous la possibilité de contribuer à la collecte des données : ainsi chacun sur le territoire national, outre-mer inclus, peut alimenter cette immense base de données. La base de données sur les complaintes criminelles, quant à elle, donne accès, grâce au travail de Jean-François Heintzen, à plusieurs centaines de crimes commis en France métropolitaine entre 1870 et 1939, pour lesquels une ou plusieurs complaintes ont été publiées. Les utilisateurs peuvent également contribuer à cette base de données en signalant et en envoyant une copie d'une feuille de chanson avec les paroles d'une complainte criminelle absente de notre corpus. Cet outil est un bel exemple, notamment, de publication de données historiques 
enrichies d'un travail de valorisation par le biais d'articles, d'expositions, de conférences chantées et de manifestations scientifiques ${ }^{15}$, de mutualisation des données dont Marin Dacos déplorait en 2013 la place de "parent pauvre de l'activité historique ${ }^{16}$ ».

\section{Les outils}

Le musée propose enfin dans sa dernière section «Outils » des bases de données de référence permettant de débuter une recherche (bibliographie sur l'histoire de la justice de 1789 à 2011, guide des archives judiciaires et pénitentiaires de 1800 à 1958). Cette section a également vocation à accueillir des bases de données produites par des chercheurs souhaitant partager le résultat de leurs travaux.

\section{La revue hypermédia Criminocorpus}

19 En complément du site musée et en liaison hypermédia avec ses ressources, la revue Criminocorpus est une publication scientifique dédiée à l'histoire de la justice, des crimes et des peines hébergée par la plate-forme OpenEdition Journals. À la différence du site musée, le contenu et la forme de la revue offrent moins de possibilités de restitution plurielle. Les auteurs peuvent y soumettre des articles et des comptes rendus d'ouvrages évalués par le comité de rédaction de la revue. Ils peuvent également proposer des dossiers thématiques ou bien alimenter un des dossiers déjà en ligne. La revue Criminocorpus n'est pas une publication périodique réalisant des numéros clos. Elle entend procéder par accumulation et publie au fil de l'eau les articles acceptés en les insérant dans des dossiers thématiques afin d'améliorer la cohérence d'accès pour les lecteurs. La revue publie également des actes de colloques et de journées d'études. Les articles sont associés, autant que faire se peut, aux sources mobilisées par les auteurs. Cette association est rendue possible par la publication simultanément de tout ou partie de ces documents sources (hébergés dans le musée Criminocorpus) ou par l'insertion dans le corps de l'article de liens hypertexte vers les sources disponibles sur internet.

\section{Le blog d'actualités}

Les actualités sur l'histoire de la justice sont diffusées via le carnet de recherche de Criminocorpus. Ce blog, hébergé par la plate-forme Hypothèses, permet de signaler des informations dans le domaine de la recherche, de l'édition et des manifestations culturelles en matière d'histoire de la justice, des crimes et des peines. Notre lettre d'informations, avec plus de 2500 abonnés, et les réseaux sociaux (Facebook et Twitter) permettent aussi de relayer ces informations auprès d'un public peut-être un peu plus large que celui du carnet de recherche quoique ce dernier soit un des plus fréquentés sur la plate-forme Hypothèses. Cependant, nous ne sommes toujours pas véritablement satisfaits de la diffusion des productions publiées sur Criminocorpus. Il ne s'agit pas d'un problème de fréquentation de notre plate-forme puisque nous comptons en moyenne 60000 visites par mois. Notre travail est connu au sein du cercle de la recherche mais trop peu à notre goût auprès des amateurs ou des professionnels de la justice qui pourtant découvrent souvent lors de nos rencontres (ateliers, séminaires, tournages, etc.) cet outil avec curiosité et intérêt. De même, nous sommes également préoccupés 
par la difficulté d'accompagner le lecteur qui ne vient pas de la recherche et qui peut être freiné par certains contenus spécialisés et pointus. C'est pourquoi nous avons initié depuis plusieurs années une réflexion sur cette question - en imaginant par exemple des parcours via des visites thématiques - et collaboré depuis quelques mois avec Emmanuelle Papinot, docteure en ergonomie cognitive sous la direction d'André Tricot (université Toulouse II - Jean-Jaurès), afin de faciliter l'accès aux contenus du site en fonction des attentes des utilisateurs.

Comme le précise Antoine Prost :

«L'histoire n'est pas la propriété des historiens. [...] La méthode historique n'a rien de spécifique. Il y a une méthode critique pour interpréter les documents, mais l'historien explique de la même façon que le témoin raconte l'accident qu'il vient de voir. L'avance des historiens sur un sujet donné ne vient pas de leur supposée maîtrise d'une méthode inaccessible au profane; elle tient au travail qu'ils ont accumulé, aux connaissances qu'ils ont lentement amassées. Cela leur permet de valider certaines affirmations, certains choix de documents ${ }^{17}$. »

Travailler en collectif, avec des chercheurs mais aussi des professionnels issus d'autres disciplines qui ne sont pas uniquement du monde de la recherche (archivistes, conservateurs, professionnels du monde de la justice, réalisateurs, etc.) ou avec des amateurs intéressés par cette thématique et aux connaissances complémentaires, est indispensable pour continuer à développer notre projet ${ }^{18}$. Ainsi, dans cette démarche, collaborer avec ces intervenants est un atout non négligeable car l'historien n'a pas forcément la main sur les sensibilités locales, et il va pouvoir ainsi apporter une contextualisation, replacer dans un contexte plus large les questionnements émanant de la société. Encore une fois les liens entre histoire et mémoire s'invitent dans notre réflexion:
« La différence majeure entre l'histoire et la mémoire ne réside pas dans la méthode ou dans le rapport aux archives. Elle se situe dans le type de questionnement adressé au passé. Les producteurs de mémoire ont surtout le souci de "sauver de l'oubli", ou de réhabiliter, les individus et les groupes qui ont leur faveur. Alors que le rôle de l'historien consiste à élaborer des questionnements qui lui permettront de mieux comprendre, voire d'expliquer, le passé, avec l'espoir que cela puisse aider les hommes d'aujourd'hui à "mieux vivre", comme disait déjà Marc Bloch ${ }^{19}\left[. . .{ }^{20}\right.$. »

Le projet Criminocorpus nous a obligés à développer des compétences pour lesquelles nous ne sommes pas formés classiquement dans nos cursus en tant que chercheurs, «à apprivoiser de nouveaux savoir-faire ». Cette expression est empruntée à l'article de Laure Blévis et Claire Zalc qui expliquent après avoir dirigé l'exposition «1931, les étrangers au temps de l'Exposition coloniale » :

«Sortir de la tour, se frotter au présent, répondre aux exigences cumulées de la mise en espace et de la mise en regard du public sont autant de prises de risques. Notre engagement dans ce projet a consisté, pour une part, à apprivoiser de nouveaux savoir-faire; il a, dans le même temps, contribué à conforter le nôtre. Il nous a permis de renforcer nos convictions quant aux manières d'écrire mais aussi de montrer le passé, d'expliciter nos fondamentaux, de faire preuve d'inventivité ${ }^{21}$.»

24 Les historiens ont rarement la charge de concevoir de bout en bout une exposition : ils sont le plus souvent associés comme experts validant la qualité des contenus ${ }^{22}$. Publier 
sur une plate-forme numérique, imaginer de nouvelles formes de récit hypermédia, de nouveaux modes de visualisation des données, accompagner les projets des collègues jusqu'à leur mise en ligne tels des passeurs et échanger avec les informaticiens supposent aussi un minimum de connaissances dans le domaine des humanités numériques et de s'interroger quant aux incidences sur le travail d'écriture de l'historien ${ }^{23}$. Ce profil de «cyberhistoriens" travaillant en collectif peut parfois intriguer, déstabiliser car nous assistons bien à un changement possible dans les pratiques et les savoir-faire du métier d'historien ${ }^{24}$. L'essentiel pour les historiens, dans ce tournant numérique, est de porter les enjeux de connaissance dans la transmission des contenus. Le numérique constitue une réelle opportunité pour mieux appréhender et restituer la complexité de l'histoire. C'est à l'historien qu'il revient de veiller à ce que cette opportunité ne soit pas gâchée au profit des mirages du technologisme.

\section{BIBLIOGRAPHIE}

AZÉMA Jean-Pierre, BADINTER Élisabeth, BECKER Jean-Jacques et al., « Liberté pour l'histoire », Libération, éd. numérique, 13 décembre 2005. [URL : http://www.liberation.fr/societe/ 2005/12/13/liberte-pour-1-histoire_541669]

BLÉVIS Laure, ZALC Claire, « Comment “mettre l'histoire en musée” : retour sur l'exposition “1931, les étrangers au temps de l'Exposition coloniale" ", La vie des idées, éd. numérique, 26 janvier 2009. [URL : http://www.laviedesidees.fr/Comment-mettre-l-histoire-en-musee.html]

BLOCH Marc, Apologie pour l'histoire ou Métier d'historien, Paris, Armand Colin, 2002 (1949).

BURGESS Joanne, «L'historien, le musée et la diffusion de l'histoire », Revue d'histoire de l'Amérique française, vol. LVII, $n^{\circ} 1,2003$, p. 33-44.

COMITÉ DE VIGILANCE FACE AUX USAGES PUBLICS DE L'HISTOIRE, « Manifeste du Comité de vigilance face aux usages publics de l'histoire », éd. numérique. [URL : http://cvuh.blogspot.fr/2007/02/ manifeste-du-comite-de-vigilance-face.html]

DAcos Marin, «Cyberclio : vers une cyberinfrastructure au cœur de la discipline historique », dans Clavert Frédéric, Noiret Serge (dir.), L'histoire contemporaine à l'ère contemporaine, Berne, Peter Lang, 2013, p. 29-41.

DELACROIX Christian, « Demande sociale et histoire du temps présent : une normalisation épistémologique? », Espaces temps, nº 84-86, 2004, p. 106-119.

DELALANDE Nicolas, VINCENT Julien, « Portrait de l'historien-ne en cyborg », Revue d'histoire moderne et contemporaine, vol. LVIII-IV bis, $\mathrm{n}^{\circ}$ 5, 2011, p. 5-29.

FEBVRE Lucien, « Vers une autre histoire », dans id., Combats pour l'histoire, Paris, Armand Colin, 1992 (1952), p. 437.

MARROU Henri-Irénée, « Tristesse de l'historien 》, Vingtième siècle : revue d'histoire, nº 45, 1995

(1939), p. 109-132. 
NOIRIEL Gérard, « Histoire, mémoire, engagement civique », Hommes et migrations, $\mathrm{n}^{\circ}$ 1247, 2004, p. 17-26.

NOIRIEL Gérard, « Naissance du métier d'historien », Genèses : sciences sociales et histoire, n 1,1990 , p. 58-85.

PAPINOT Emmanuelle, «Criminocorpus, musée d'Histoire de la justice. Quels publics ? Quels usages ? Quelle ergonomie ? ", rapport de recherche, éd. numérique, Paris, CLAMOR, 2018. [URL : https:// hal.archives-ouvertes.fr/hal-01800523]

PROST Antoine, «Comment l'histoire fait-elle l'historien? ", Vingtième siècle : revue d'histoire, $\mathrm{n}^{\circ}$ 65, 2000, p. 3-12.

PROST Antoine, "Transmettre l'histoire ", dans Chemins d'accès : quelle(s) médiation(s) pour l'histoire? $9^{e}$ rencontres des services éducatifs de musées, bibliothèques, archives et théâtres, éd. numérique, Paris, BNF, 2011. [URL : http://classes.bnf.fr/actes/9/Prost.pdf]

RENNEVILLE Marc, SANCHEZ Jean-Lucien, VICTORIEN Sophie, «Criminocorpus : un projet numérique pour l'histoire de la justice ", Digital humanities quarterly, éd. numérique, vol. XII, $n^{\circ} 1,2018$. [URL : http://www.digitalhumanities.org/dhq/vol/12/1/000365/000365.html]

RYGIEL Philippe, Historien à l'âge numérique, Villeurbanne, Presses de l'ENSSIB, 2017.

\section{NOTES}

1. https://www.huma-num.fr/

2. https://criminocorpus.org/fr/

3. https://journals.openedition.org/criminocorpus/

4. https://criminocorpus.hypotheses.org/

5. G. Noiriel, « Naissance du métier d'historien », p. 82.

6. C. Delacroix, «Demande sociale et histoire du temps présent: une normalisation épistémologique?».

7. Comité de vigilance face aux usages publics de l'histoire, «Manifeste du Comité de vigilance face aux usages publics de l'histoire ".

8. Voir le texte de la pétition, J.-P. Azéma, É. Badinter, J.-J. Becker et al., « Liberté pour l'histoire ".

9. L. Febvre, « Vers une autre histoire », p. 437.

10. A. Prost, « Comment l'histoire fait-elle l'historien ? », p. 5-6.

11. H.-I. Marrou, « Tristesse de l'historien », p. 130.

12. A. Prost, « Transmettre l'histoire », p. 3.

13. Pour en savoir plus sur l'évolution de Criminocorpus et les étapes de ce projet, voir M. Renneville, J.-L.Sanchez, S. Victorien, «Criminocorpus : un projet numérique pour l'histoire de la justice ".

14. E. Papinot, «Criminocorpus, musée d'Histoire de la justice. Quels publics? Quels usages? Quelle ergonomie?».

15. Ce projet lancé par Jean-François Heintzen a été mis en ligne en collaboration avec le CLAMOR. Il a donné lieu à la constitution d'un comité scientifique réunissant des 
chercheurs issus de différentes disciplines chargés de travailler sur ces sources encore peu explorées et pourtant si riches et de préparer un colloque sur cette thématique organisé en 2019 dont les actes sont disponibles dans notre revue.

16. M. Dacos, "Cyberclio: vers une cyberinfrastructure au cœur de la discipline historique ", p. 32.

17. A. Prost, « Transmettre l'histoire », p. 7.

18. Nous nous appuyons sur la définition de Gérard Noiriel quand nous utilisons l'expression d'historien professionnel : « Un "historien", au sens actuel du terme, est un "professionnel" de l'histoire, c'est-à-dire un individu pour qui la recherche historique est un métier, qui est par conséquent formé et rémunéré comme tel.» (G. Noiriel, « Naissance du métier d'historien », p. 58.)

19. M. Bloch, Apologie pour l'histoire ou Métier d'historien.

20. G. Noiriel, « Histoire, mémoire, engagement civique », p. 19.

21. L. Blévis, C. Zalc, «Comment "mettre l'histoire en musée": retour sur l'exposition “1931, les étrangers au temps de l'Exposition coloniale" ".

22. J. Burgess, «L'historien, le musée et la diffusion de l'histoire ».

23. Sur cette question, voir notamment $\mathrm{P}$. Rygiel, Historien à l'âge numérique.

24. N. Delalande et J. Vincent, « Portrait de l'historien-ne en cyborg ».

\section{RÉSUMÉS}

Conçu en 2003 par des historiens associés à des archivistes et des documentalistes, Criminocorpus est né d'une intention historiographique et de diffusion du savoir historique qui a marqué le paysage numérique français par sa logique thématique centrée sur l'histoire de la justice, des crimes et des peines. Espace d'expérimentations numériques open source en libre accès, le site web initial s'est enrichi de nouvelles fonctionnalités, d'un blog externe et d'une revue. En 2016, la plate-forme a produit le premier musée numérique d'Histoire de la justice, des crimes et des peines. Nous proposons de retracer dans cet article notre démarche de transmission auprès de plusieurs lectorats identifiés (historiens, professionnels de la justice, étudiants...) et, au-delà, de toute personne intéressée.

\section{AUTEURS}

\section{MARC RENNEVILLE}

Directeur de recherche au CNRS, Centre pour les humanités numériques et l'histoire de la justice (CLAMOR, UMS 2637)

\section{JEAN-LUCIEN SANCHEZ}

Chargé d'études en histoire, direction de l'Administration pénitentiaire, Laboratoire de recherche et d'innovation, ministère de la Justice 


\section{SOPHIE VICTORIEN}

Ingénieure de recherche au CNRS, Centre pour les humanités numériques et l'histoire de la justice (CLAMOR, UMS 2637) 


\title{
Transmission numérique des savoirs : quel type de vidéos, quel rôle des structures publiques?
}

\author{
Alexandre Moatti
}

1 Alors au cabinet de la ministre de la Recherche (Claudie Haigneré), nous avions organisé en mars 2004 la $1^{\text {re }}$ Fête de l'internet scientifique ${ }^{1}$ (sur le modèle de la Fête de l'internet, gérée elle depuis 1997 par des associations d'usagers). Ce fut la seule, mais elle marquait une prise de conscience publique de l'internet comme outil de transmission des savoirs. En même temps était inauguré par le ministère le site science.gouv.fr (disparu en 2014), qui recensait divers sites publics et académiques de vulgarisation de qualité - ainsi qu'était lancé le site mariecurie.science.gouv.fr, conçu par l'université Paris VIII et soutenu par le ministère afin de promouvoir des contenus francophones de qualité lors du $100^{\mathrm{e}}$ anniversaire du prix Nobel des Curie (décembre 2003²).

connaît la suite en matière d'internet scientifique, qu'il n'y a pas lieu de décrire là ; après les sites et portails internet, avec le web 2.0 apparurent les blogs de sciences, d'abord dans le monde anglophone, puis dans le monde francophone (à partir de 2005³). La troisième phase - toujours en cours - fut celle des vidéos scientifiques, avec l'immense essor pris par ce média internet: en matière d'action publique, ce fut l'arrivée bienvenue, en 2010, d'Universcience.tv (Cité des sciences/ Palais de la découverte), venant là aussi combler un manque en matière de vidéos francophones de vulgarisation; puis l'arrivée des youtubeurs vulgarisateurs (sciences, histoire, etc.), dans le monde anglophone (vers 2010) puis francophone (vers 2013).

Pourtant - et là l'histoire est moins connue -, l'action publique avait pris une longueur d'avance en matière de transmission des savoirs, y compris par vidéo : le site Canal- $U^{4}$, "la web TV des universités", avait été créé dès 2000 , dans une optique fédératrice de diverses initiatives universitaires de cours et conférences vidéo ${ }^{5}$. Comme souvent en France, de bonnes idées et des projets numériques précurseurs existent, avant que l'essai soit transformé ailleurs (un autre exemple serait Gallica, créé par la BNF bien avant «l'affaire » Google Books vs Bibliothèque numérique européenne ${ }^{6}$ ). Intégrons aussi 
dans cette vista pionnière l'initiative du philosophe Yves Michaud, lors de la mission An 2000 (conduite par Jean-Jacques Aillagon) : l'Université de tous les savoirs (UTLS), 365 conférences sur l'état du savoir scientifique, enregistrées, filmées et mises en ligne chaque jour de l'année 2000, bientôt suivies de 620 conférences « UTLS-La suite ${ }^{7}$ » (dont « UTLS au lycée »), de 2001 à 2013.

4 Après ce rappel historique sommaire, que dire de nos jours de l'action publique en la matière, alors que la notion même d'action publique semble à présent galvaudée, et que la «matière » est maintenant en vrac sur internet, émanant de concepteurs et diffuseurs de tous ordres? Comme le décrivait Yves Michaud lui-même, dans sa conférence de clôture de quatorze années d'aventures :

«La massification a noyé notre projet dans un raz-de-marée d'offres [...], un changement complet de mode de sélection de la connaissance s'est opéré : au début des années 2000 il s'agissait de trier puis diffuser - à présent il s'agit de publier puis trier." "

5 Tout acteur public, qu'il s'agisse de Canal-U ou d'UTLS les précurseurs, ou d'Universcience.tv par la suite, n'a pu que constater ce déferlement de contenus de transmission du savoir, que ce soit via les youtubeurs individuels (et... individualistes? En tout cas en conformité avec la mise en scène que chacun fait de soi sur internet) ; ou via des initiatives plus organisées et internationales, comme les conférences TED (Technology, entertainment and design) ou TEDx ${ }^{9}$ qui, comme UTLS, conservent le lien avec une conférence physique.

6 Ici n'est pas non plus le lieu de décrire et d'analyser ces multiples chaînes vidéo. Néanmoins le responsable public, ou l'enseignant-chercheur, pourrait assurer un suivi et une évaluation de ces divers canaux, et ce en appui de ses étudiants, ou du grand public, ou des enseignants du secondaire. Même si la qualité moyenne est tout à fait acceptable, certaines chaînes YouTube, que ce soit en sciences exactes ou en sciences humaines, sont meilleures que d'autres; et même certaines chaînes de bon niveau peuvent perdre en qualité dans le temps. La recherche publique pourrait s'intéresser plus étroitement à l'évaluation de ces chaînes; et l'action publique en matière d'éducation nationale aussi.

7 Mais c'est sur une critique plus incisive de l'action publique actuelle en matière de diffusion des savoirs que nous souhaiterions centrer notre propos ; en distinguant deux pôles, l'action publique dépendant du ministère de la Culture, et celle dépendant de l'Enseignement supérieur et de l'Éducation nationale. Nous prendrons à cet effet quelques exemples, tirés de l'action publique dans l'orbite du ministère de la Culture.

Le premier exemple concerne les émissions culturelles et scientifiques de Radio France (France Culture, France Inter). Bien que financées sur fonds publics, elles ne sont pas disponibles sans limite de temps, mais téléchargeables pendant une durée d'un an, et en streaming pendant trois ans; au-delà, elles ne sont plus accessibles car soumises à droits d'auteurs (de l'animateur, voire des intervenants ${ }^{10}$ ). Les accords passés par Radio France avec les sociétés de droits d'auteurs ne sont pas rendus publics : mais, dans le 
budget de l'établissement public, la dépense relative aux sociétés de droits (pour la diffusion de l'émission elle-même et pour ces téléchargements, ou podcasts) se monterait à 35 millions d'euros par an. Il est dommageable que le système de coproduction, mis en place à la télévision française vers 1995 autour des émissions d'entertainment, ait été ainsi étendu aux émissions radiophoniques du savoir. De fait, le paiement en droits d'auteurs constitue une économie importante en charges sociales pour l'établissement public, permettant d'améliorer à plus bas coût la rémunération des animateurs d'émission ${ }^{11}$, entretenant un régime de "starification»: ce dernier phénomène est évidemment moins sensible pour les présentateurs d'émissions culturelles que pour les stars du divertissement télévisuel, mais cette dérive de rémunération vers un système de droits d'auteurs a cette conséquence inattendue, et problématique, d'une limitation de l'accès numérique à la connaissance... Il y a là une forme de déresponsabilisation de l'institution publique à transférer sur une gestion collective de droits ce qui relève d'une stratégie et d'une chaîne de valeur au cœur de son métier, à savoir rendre disponibles de manière permanente des savoirs l'éducation étant une des missions du service public audiovisuel.

9 L'accès aux documentaires culturels des chaînes de télévision publique illustre aussi cette dérive. Qu'il y ait coproduction avec des auteurs et des producteurs (et prise de risque associée) est sans doute là plus dans l'ordre des choses; que ces documentaires puissent être achetés sur un site France Télévisions (par exemple Pluzzvad, fondu en 2017 dans le site France.tv dépendant du groupe public), aussi. Cependant ces documentaires (« émissions de stock ») sont là noyés dans l'intégralité de l'offre France Télévisions (divertissement compris, ou "émissions de flux ») : il pourrait être utile de les rendre disponibles (y compris à la vente) de manière différenciée sur internet, et beaucoup plus visible. Ces documentaires sur Churchill, sur Einstein, sur la Première Guerre mondiale doivent-ils voisiner avec les séries télé ? Comment mieux faire connaître ce type de productions? Il est enfin une autre catégorie de documentaires de télévision publique, ne figurant pas sur le site France.tv, même à l'achat, et de la société de production de laquelle on doit alors rechercher le site "à la main »: et là le documentaire n'est pas nécessairement à la vente, ni en $\mathrm{CD}$, ni en téléchargement ${ }^{12} \ldots$ Vient alors naturellement la question corrélative: pourquoi ne pas rendre ces documentaires éducatifs et culturels disponibles de manière gratuite, quitte à ce que ceci soit prévu dès le départ dans les contrats avec les chaînes publiques ? Certes ces documentaires pourraient être à nouveau vendus par leurs producteurs à des chaînes francophones (par exemple Canada, Suisse, Belgique...), ou rediffusés, ou rachetés et traduits, ou vendus en DVD. Mais s'agit-il de cas fréquents, de recettes importantes pour ces producteurs de documentaires - dont souvent la chaîne publique est d'ailleurs coproductrice? Une télévision étrangère renoncerait-elle à l'achat d'un documentaire pour la simple raison qu'il est déjà disponible sur internet? Ce n'est pas certain.

Le deuxième exemple, toujours dans le domaine des politiques publiques du numérique culturel, a trait aux vidéos de l'Institut national de l'audiovisuel (INA), établissement dépositaire des archives de l'audiovisuel public. Un certain nombre d'émissions culturelles de grande valeur (par exemple les émissions de l'ex-ORTF, à une époque où l'office public était son propre producteur - ce vers quoi l'on pourrait revenir pour les émissions scientifiques et culturelles) sont disponibles de manière payante sur le site de l'INA : ainsi il vous en coûtera 2,99 euros pour visionner un remarquable débat d'une heure entre Claude Lévi-Strauss et François Jacob, au cours duquel les deux professeurs au Collège de France s'entretiennent, en janvier 1972, de «la pensée sauvage » décrite 
par l'ethnologue ${ }^{13}$. Là aussi pose problème le fait que des émissions qui sont pleinement propriété publique (via l'ORTF) et dont la numérisation a été financée par le grand emprunt (investissements d'avenir, financement public) soient payantes pour l'internaute - qui en tant que contribuable en viendrait à payer une troisième fois pour le même contenu... Le rapport d'activité de l'INA est peu disert sur ces ressources propres: nous avons évalué à 250000 euros par an (sur un budget total de fonctionnement de 125 millions d'euros ${ }^{14}$ ) la part de ces ressources - ce que l'on peut appeler une "politique des picaillons" (c'est-à-dire de très faibles revenus), par rapport à l'enjeu que constitue l'accès libre et gratuit à ces vidéos publiques du savoir, et le rayonnement ainsi apporté à l'institution publique ${ }^{15}$.

11 De manière connexe, liée aussi à l'INA, vient une question que nous avons mise en exergue en début d'article : une institution publique doit-elle obligatoirement valoriser ses contenus sur YouTube (filiale de Google depuis 2005) ? Sans répondre à cette question, pour laquelle les jeux semblent déjà faits, nous donnerons deux effets pervers de cet état de fait. L'un est issu de notre expérience propre, celle de notre web TV universitaire d'humanités et de culture générale CultureGnum. $\mathrm{fr}^{16}$, et nous ramène à Radio France : pour être intégré au portail de webconférences universitaires de France Culture, il est nécessaire à ce jour (pour des raisons informatiques, ou d'uniformisation paresseuse) d'avoir ses vidéos sur YouTube! Ainsi, d'un producteur public (notre chaîne CultureGnum) à un autre (le portail de conférences France Culture), il est nécessaire de passer par YouTube, donc par les GAFA ${ }^{17}$. De la même manière, relevons qu'un producteur de contenus culturels publics (comme l'INA) aura tendance à être plus attentif à son site YouTube qu'à son site propre, notamment à cause du compteur de vues qui est l'élément de reconnaissance sur internet !... Sans (trop) forcer le trait, on peut dire que le compteur YouTube des abonnés a un caractère addictif pour un producteur vidéo (public ou non) - c'est une redoutable arme de YouTube pour agréger sur sa plate-forme les producteurs de contenus.

12 Et l'action publique en matière d'enseignement supérieur, dans tout ça? Le sujet est ténu. Il existe des vidéastes isolés dans des laboratoires qui font un travail remarquable ${ }^{18}$; nous avons aussi mentionné notre chaîne CultureGnum.fr (FMSH, dans le cadre de Canal-U). Existent bien évidemment des web TV plus spécifiques liées à un organisme de recherche donné ${ }^{19}$, sachant qu'elles sont parfois plus attachées à l'actualité et à la politique de communication de l'organisme (y compris dans les annonces de résultats scientifiques) qu'à une transmission des savoirs, qui elle est peutêtre moins spectaculaire et plus laborieuse.

Il n'y a pas d'action explicite de soutien à la vidéo de diffusion des connaissances de la part du ministère de l'Enseignement supérieur. Le ministère de la Culture, via le Centre national du cinéma $(\mathrm{CNC})$, finance quant à lui depuis 2017 des youtubeurs ${ }^{20}$, via des programmes de soutien allant de 30000 à 50000 euros par an pour les vidéastes sélectionnés; dans une optique libérale, on peut s'étonner de pareille utilisation des fonds publics (ce serait en fait l'adaptation aux «nouveaux» médias des aides publiques au cinéma et aux documentaires); dans une optique antilibérale, on peut s'étonner que ces aides passent par l'utilisation d'un média appartenant aux acteurs monopolistiques de l'internet... En tout état de cause, remarquons la disproportion des 
moyens publics mobilisés : on peine à trouver, en tant qu'acteur public de la diffusion de la connaissance (comme CultureGnum), un soutien public de fonctionnement, alors que les établissements publics du ministère de la Culture disposent de crédits destinés à des vidéastes privés YouTube - nous ne portons pas là de jugement de valeur sur ces derniers, mais nous notons la dissymétrie.

La situation générale est que chaque organisme de recherche, chaque université, chaque grand établissement (Collège de France, Académie des sciences, etc.) développe ses vidéos sur son site propre, chacun considérant que YouTube est le point de ralliement, où l'on peut trouver toutes ces vidéos. Nous plaidons cependant pour une action publique concertée (Culture et Enseignement supérieur) autour d'un portail de vidéos de diffusion de la connaissance, donnant accès aux divers sites publics. Dans son bilan de 2013, parfois amer à propos de la masse des contenus internet, Yves Michaud lui aussi estime - touche positive - qu'il y a encore de la place pour une stratégie éditoriale d'orientation, au sein d'un "portail de la culture francophone ». Certes, il convient d'être vigilant, les stratégies proclamées avec le portail Bibliothèque numérique européenne (2005) ayant plutôt échoué ; la même velléité revient pourtant sous diverses formes dans plusieurs domaines, comme "l'Airbus du numérique », ou le « Netflix à la française ${ }^{21}$ »... Entre deux voies, celle d'une action publique à caractère bureaucratique amenant à ce genre d'impasses, et celle d'une action publique s'en remettant uniquement à YouTube pour assurer la fonction de portail de vidéos, il existe peut-être une voie médiane, celle d'un portail léger assurant l'indexation de l'ensemble des contenus culturels publics, audio et vidéo : podcasts France Culture, vidéos INA dans leur intégralité, chaînes des organismes de recherche, Collège de France, Canal-U bien sûr... Le fonctionnement en silos des diverses institutions éducatives et culturelles chacune cultivant son jardin - est un réel handicap sur internet : y faire masse, et y faire connaître un portail unique d'émissions culturelles de télé et de radio, est un défi à relever par l'action publique, au bénéfice de l'accès numérique à la connaissance.

\section{BIBLIOGRAPHIE}

BENHAMOU Françoise, Les dérèglements de l'exception culturelle : plaidoyer pour une perspective européenne, Paris, Le Seuil, 2006

BRATTON Benjamin, « We need to talk about TED », The Guardian, éd. numérique, 30 décembre 2013. [URL : https://www.theguardian.com/commentisfree/2013/dec/30/we-need-to-talk-aboutted]

« France TV, TF1 et M6 s'allient pour créer un Netflix à la française », Le Parisien, éd. numérique, 15 juin 2018. [URL : https://www.leparisien.fr/culture-loisirs/tv/france-tv-tf1-et-m6-s-allientpour-creer-un-netflix-a-la-francaise-15-06-2018-7773741.php]

LIÈVRE Hervé, « La web télévision, une nouvelle forme de diffusion des savoirs ? L'exemple de Canal- $U$ », Perspectives documentaires en éducation, $\mathrm{n}^{\circ}$ 62, « Diffusion des résultats de la recherche : une nouvelle donne numérique ", 2005, p. 101-107. 
"Les youtubeurs vont pouvoir bénéficier des aides du CNC », L'Express, éd. numérique, 3 octobre 2017. [URL : https://lexpansion.lexpress.fr/high-tech/les-youtubeurs-vont-pouvoir-beneficierdes-aides-du-cnc_1949091.html]

MOATTI Alexandre, Au pays de Numérix, Paris, Presses universitaires de France, 2015.

MOATTI Alexandre, «Bibliothèque numérique européenne : de l'utopie aux réalités ", Annales des mines : réalités industrielles, 2012, p. 43-46.

\section{NOTES}

1. On en trouve une trace sur les archives gouvernementales du site internet.gouv.fr (http://archives.internet.gouv.fr/archives/rubrique7809-7659.html) et dans la presse, par exemple https://bfmbusiness.bfmtv.com/01-business-forum/les-sciencessinvitent-a-la-fete-de-linternet-238349.html

2. Le site, conçu au Laboratoire d'évaluation et de développement pour l'édition numérique (LEDEN, Paris VIII / MSH Paris-Nord) par la Pr Ghislaine Azémard et ses étudiants, n'existe plus en tant que tel; il continue à servir cependant, depuis 2005, de support multimédia à une exposition itinérante, en France et dans le monde (Chine, Colombie, océan Indien), par exemple début 2018 au Panthéon à Paris (voir http:// leden.org/exposition-marie-curie-femme-de-science/). Nous souhaitons rendre hommage à Jean-Pierre Dalbéra, alors à la tête de la mission Recherche et technologie (MRT) du ministère de la Culture, qui avait pavé la voie avec ses remarquables sites anniversaires de personnalités et d'événements culturels.

3. Voir par exemple l'association Café des sciences (https://www.cafe-sciences.org/), réunissant depuis 2005 des blogueurs scientifiques (nous avons tenu, de 2006 à 2014, un blog de mathématiques et de physique adhérant à cette association).

4. https://www.canal-u.tv/

5. Un historique de Canal-U à ses débuts est donné par l'un de ses fondateurs : H. Lièvre, "La web télévision, une nouvelle forme de diffusion des savoirs? L'exemple de Canal$U$ ». Nous remercions Hervé Lièvre de nous avoir fait connaître cette référence.

6. Pour un historique critique du sujet "Google défiant l'Europe ", voir A. Moatti, Au pays de Numérix ; id., "Bibliothèque numérique européenne : de l'utopie aux réalités ».

7. Ces conférences ont longtemps figuré sur le site du Monde, à la suite d'un partenariat noué avec cet organe de presse. Signe des temps, elles en ont disparu, mais elles sont bien heureusement conservées sur le site Canal-U, susmentionné : https://www.canalu.tv/producteurs/universite_de_tous_les_savoirs

8. Y. Michaud, "L'université de tous les savoirs : bilan d'une aventure », conférence du 29 septembre 2013 (https://www.canal-u.tv/video/universite_de_tous_les_savoirs/ 1_universite_de_tous_les_savoirs_bilan_d_une_aventure.12936).

9. On trouve une intéressante critique de la démarche TED/TEDx par B. Bratton, « We need to talk about TED ». Le chroniqueur y dénonce la place trop importante faite non à la transmission du savoir, mais au témoignage personnel, aux discours du "bien-être », voire à la " placebo science ", dans des discours parfois très simplificateurs, appuyés sur le marketing et le technoprophétisme. 
10. Les intervenants, par exemple des universitaires dans des émissions de France Culture, savent peu qu'ils peuvent déclarer leur passage à une société de droits d'auteurs (comme la Société civile des auteurs multimédia) et être rémunérés dans le cadre des accords entre Radio France et les sociétés de droits (environ 80 euros pour une heure d'interview, ce qui n'est pas négligeable). Ceci est peu connu hors les journalistes et intervenants professionnels, et pour cause puisqu'un seul et même montant est à se partager en fin d'année (le moins de personnes se déclarent, le plus important est le taux horaire pour les déclarants habituels).

11. Ce système de droits d'auteurs pour les présentateurs permet de diminuer les charges sociales, comme le régime des intermittents permet de diminuer - pour l'établissement public - les charges sociales relatives aux techniciens des mêmes émissions, ce qui a souvent été dénoncé. Pour Françoise Benhamou, spécialiste de l'économie de la culture, "la législation sociale pousse à cette dérive, les cotisations sociales étant bien moindres pour un auteur que pour un salarié » (F. Benhamou, Les dérèglements de l'exception culturelle : plaidoyer pour une perspective européenne).

12. Nous donnerons un exemple, à propos d'un excellent documentaire, pas de télévision nationale, mais en partie importante financé sur fonds publics (CNC, Télé Lyon Métropole, région Rhône-Alpes, direction de l'Architecture du ministère, etc.) : Firminy: le maire et l'architecte (2007, 54 minutes, http://www.film-documentaire.fr/ 4DACTION/w_fiche_film/26666_1); nous n'avons pas pu trouver ce documentaire à l'achat.

13. https://madelen.ina.fr/programme/la-logique-du-vivant-francois-jacob-rencontreclaude-levi-strauss (série « Un certain regard »).

14. Nous avons détaillé dans Au pays de Numérix notre calcul à partir des données disponibles (INA rapport d'activités, 2013). Depuis 2016, le rapport d'activités ne permet plus de faire ce genre d'extrapolations. On peut néanmoins lire sur le site de la direction du Budget (https://www.performance-publique.budget.gouv.fr/sites/ performance_publique/files/farandole/ressources/2016/rap/pdf/

DRGPGMPGM845.pdf), programme $n^{\circ} 845$, INA, que : 1) «L'arrêt d'une facturation d'un minimum garanti sur la plate-forme YouTube impacte le résultat de $89 \mathrm{~K} €$ par rapport à l'année 2015 »; 2) « Les recettes sur l'activité du online sont baisse de $10 \mathrm{~K} €$ par rapport à 2015 ", ce qui ne laisse guère imaginer une augmentation sensible des ressources propres liées aux abonnements des internautes.

15. Cette " politique des picaillons » est aussi à l'œuvre dans l'absence de publication d'une iconographie libre de droits sur internet, émanant de collections patrimoniales : la même pusillanimité existe dans les institutions muséales et mémorielles publiques en France, par comparaison à d'autres pays (voir A. Moatti, Au pays de Numérix: un exemple en est la photographie de Charles de Gaulle sur Wikipédia, émanant non d'une collection française mais - clin d'œil de l'histoire - de la Bundesarchiv allemande...).

16. Nous avons créé cette chaîne www.cultureGnum.fr en octobre 2016, dans le cadre de Fondation Maison des sciences de l'homme (FMSH, Paris).

17. Rappelons que c'est par cet acronyme que sont désignés les grands acteurs numériques quasi monopolistiques : Google, Amazon, Facebook, Apple (GAFA).

18. Voir par exemple la vingtaine de vidéos du Centre d'histoire sociale des mondes contemporains (Paris I) réalisées par Jeanne Menjoulet: https://histoiresociale.cnrs.fr/films/films-chs/. 
19. Voir par exemple la chaîne Interstices de l'Institut national de recherche en informatique et en automatique (INRIA) : https://interstices.info/?s=\&fwp_type=video.

20. Voir «Les youtubeurs vont pouvoir bénéficier des aides du CNC » ainsi que, sur le site du CNC, "Fonds d'aide aux projets pour les nouveaux médias»: https:// www.cnc.fr/professionnels/aides-et-financements/nouveaux-medias-et-creationnumerique/aide-a-l-innovation/fonds-daide-aux-projets-pour-les-nouveauxmedias_191100.

21. «France TV, TF1 et M6 s'allient pour créer un Netflix à la française ».

\section{RÉSUMÉS}

Dans le cadre du thème de "La transmission des savoirs" du $143^{\mathrm{e}}$ Congrès du CTHS, nous souhaitons amorcer ici une brève histoire de la vulgarisation scientifique sur internet : avec une vingtaine d'années de recul, il s'agit déjà d'un objet historique. Ce faisant, en tant qu'acteur public ainsi qu'observateur de l'action publique dans ce domaine, nous nous demanderons quelle y est la place de cette dernière, en distinguant l'action publique liée au ministère de la Culture et celle liée au ministère de l'Enseignement supérieur. La question réductrice de notre propos et sans doute provocatrice pouvant être : « Peut-on penser une action publique hors YouTube? »...

\section{AUTEUR}

\section{ALEXANDRE MOATTI}

Chercheur associé HDR à l'université Paris-Diderot (SPHERE, UMR 7219)

Chercheur au Réseau atelier internet lyonnais (RAIL, ENSSIB / IXXI) 


\title{
Système de formation en ligne et norme de mérite : le projet TeSLA
}

\author{
Nathan De Vos
}

1 Cette communication tire sa matière de l'expansion des universités dites open universities, ou universités ouvertes, qui proposent des cursus entièrement en ligne. Ces institutions sont davantage à rapprocher des cours par correspondance que des très à la mode formations ouvertes à distance, ou MOOC (massive open online course), qui insistent sur le côté collaboratif et communautaire que rendent possible les nouvelles technologies. Les universités ouvertes, à l'inverse, proposent un apprentissage qui se veut « classique » car censé mener à l'obtention d'un diplôme équivalent à ceux reçus via un cursus en université " présentielle » tout en dépassant les restrictions en matière d'espace et de temps de ces établissements classiques.

2 Ces sources nouvelles d'enseignement sont à la recherche d'un positionnement clair, justement à mi-chemin entre les universités traditionnelles et les MOOC, autrement dit entre d'un côté des institutions jouissant d'une légitimité traditionnelle et de l'autre un mode de partage d'un savoir fluide, libre et moins ancré institutionnellement. Les universités ouvertes souhaitent en quelque sorte capter les avantages des deux modes d'enseignement en offrant la possibilité d'obtenir des diplômes institutionnalisés comme ceux qui sont obtenables dans les universités classiques mais avec la flexibilité logistique des MOOC.

Or ces universités ouvertes se heurtent à la difficulté d'organiser des évaluations pour un nombre important d'étudiants dans un contexte d'asynchronicité spatiale et temporelle (les MOOC ont moins ce problème car ils ne doivent pas spécialement mener à une certification du savoir acquis). Cette difficulté est d'abord logistique. Il est, en effet, délicat de faire en sorte que l'ensemble des étudiants se réunisse en un lieu à un moment donné pour passer un examen puisque la raison même de leur engagement dans ce type d'université est leur manque de disponibilité. Certaines universités tentent de résoudre cette contrainte via l'implantation de centres d'études (study centers) décentralisés dans les différentes régions du pays concerné, diminuant la complexité organisationnelle des examens du point de vue des étudiants, mais cette solution est peu satisfaisante car onéreuse (notamment à cause de la nécessité de louer 
des locaux uniquement en période d'examens, de payer du personnel surveillant...) et peu réaliste pour atteindre les étudiants profitant de la formation en ligne (e-learning) afin d'étudier dans une université de leur pays d'origine tout en travaillant à l'étranger.

4 La difficulté est également d'ordre pédagogique, puisque cette distance rend délicate l'analyse de la manière dont les étudiants s'approprient la matière diffusée en ligne. Excepté par le biais d'enquêtes réalisées a posteriori, les professeurs sont peu à même de savoir comment s'effectue cette appropriation, la communication entre les deux parties étant généralement limitée du fait de cette asynchronicité spatiale et temporelle. Cela est problématique pour certaines universités lorsqu'elles tentent de fournir une certification perçue, tant par les étudiants potentiels que par le marché du travail, comme aussi valable et légitime que celle de leurs concurrentes qui assurent cours et examens en présence. L'idée d'égalité des chances entre étudiants, concept normatif ayant subi de nombreuses et riches mutations au cours de l'histoire sur lesquelles nous n'insisterons pas ${ }^{1}$, constitue un argument de légitimité fondamental de l'enseignement. Mais comment envisager cette égalité, quelles que soient les nuances qu'on lui apporte, si les étudiants évoluent dans un contexte aussi flou quant à leurs conditions réelles de travail ? Il devient par exemple facultatif, dans ce type de modèle pédagogique, de réunir les étudiants en un moment donné et d'être certain qu'ils aient reçu les mêmes explications ou de leur donner la possibilité de poser des questions en même temps que les explications sont transmises.

5 De ce fait, la mise au point d'un système pouvant améliorer la fiabilité des évaluations accomplies en ligne constitue un enjeu énorme, tant pédagogiquement que commercialement. La Commission européenne s'est emparée de cette problématique en dégageant $\mathrm{du}$ financement pour la recherche et le développement de solutions d'évaluation en ligne (e-assessment). Le projet TeSLA provient de cette initiative. Il se caractérise, comme nous allons le voir, par l'intégration d'outils de surveillance biométrique.

6 Cet article débutera par une description brève du système TeSLA. Ensuite, il explicitera la méthodologie utilisée lors de notre travail de terrain ainsi que ses objectifs. Puis il démontrera en quoi le processus d'appropriation de TeSLA par ses utilisateurs diffère de l'usage tel qu'il était imaginé a priori par les concepteurs du projet et évoquera les interrogations éthiques et sociologiques qui en découlent. Enfin, une courte analyse explicitera les implications d'une telle appropriation du système en matière de mérite scolaire.

\section{Méthodologie de l'enquête}

7 Notre angle d'approche consiste avant tout à considérer les technologies telles que TeSLA comme des configurateurs sociaux. En effet, loin d'être considérée comme un objet inerte et totalement malléable par ses utilisateurs, cette technologie va rendre possible (ou empêcher) un ensemble d'actions émanant de personnes ou de groupes de personnes. Elle va attribuer des rôles, permissifs ou à l'inverse restrictifs selon les individus $^{2}$. De ce fait, un objet technologique influe sur une certaine forme d'interaction sociale. En tant qu'interface entre le professeur et ses étudiants, TeSLA convient particulièrement à ce type d'approche qui permet de garder un regard extérieur et potentiellement critique. Nous proposons par conséquent de nous focaliser sur les pratiques que nous avons effectivement observées dans le cadre de ce projet. 
8 Cette approche nécessite donc d'analyser comment les concepteurs du projet se représentent en amont les utilisateurs du système et leur usage de celui-ci. Comme l'évoque Lucy Suchman, les concepteurs du projet font des "plans", ils dressent notamment les actions concrètes que va induire l'appropriation du système. C'est une approche déterministe, du fait qu'elle postule que les utilisateurs vont respecter le principe du système et les usages qui en découlent ${ }^{3}$.

Pour ce faire, notre enquête de terrain s'est déroulée en deux temps. Le premier temps est celui des entretiens exploratoires réalisés auprès des partenaires du projet alors que celui-ci était encore à l'état d'embryon ${ }^{4}$. Ces entretiens ont permis de faire comprendre le fonctionnement global du système, mais aussi quelles étaient les idées sous-jacentes au projet. En un second temps, des entretiens avec des professeurs et des étudiants ayant expérimenté le système pendant une des périodes de test ont été menés ${ }^{5}$. Nous insisterons sur les entretiens faits auprès des professeurs pour les observations qui suivront. Les étapes de cette enquête ont toutes deux été menées sur la base d'une méthodologie semi-compréhensive inspirée de Christophe Lejeune ${ }^{6}$ et de Jean-Claude Kauffman ${ }^{7}$, autrement dit avec des questions ouvertes permettant aux répondants d'aborder le système de la manière qui avait le plus de sens pour eux.

\section{Qu'est-ce que TeSLA?}

10 TeSLA est un système d'évaluation destiné à un usage exclusivement en ligne et plus précisément un système d'authentification des apprenants. En effet, TeSLA est totalement découplé du contenu de l'apprentissage ou des évaluations, il vise uniquement à déterminer si l'étudiant effectuant des tâches sur un ordinateur est celui qu'il prétend être ou non. Il est utilisé pour authentifier l'étudiant lors d'« activités d'évaluation » au sens large (celles-ci pouvant être des examens écrits ou oraux, des rédactions, des exercices...). Comme déjà évoqué, le système permet l'asynchronicité temporelle des étudiants, ceux-ci pouvant généralement réaliser l'activité au moment où ils le souhaitent dans le respect d'un temps imparti. D'un point de vue logiciel, TeSLA prend la forme d'un module externe fonctionnant sur plusieurs plates-formes de formation en ligne largement distribuées sur le marché.

11 TeSLA se dote d'un ensemble d'outils de biométrie et d'analyse de texte pour réaliser cette authentification. Il est la combinaison des cinq instruments suivants :

- la reconnaissance faciale : l'outil fonctionne par le biais de la webcam, l'étudiant étant censé se tenir devant son ordinateur pendant la durée de l'activité. Il fonctionne en quasi continu en comparant des photos de l'étudiant prélevées à un rythme très régulier (toutes les minutes environ) avec les données de l'enrôlement ;

- la reconnaissance vocale, d'un fonctionnement similaire à la reconnaissance faciale, dédiée aux examens oraux;

- la reconnaissance de frappe au clavier : l'outil reconnaît notamment des régularités dans la manière de frapper au clavier indépendamment du contenu rédigé sur la base du principe de mémoire musculaire des doigts (par automatisme, ceux-ci pressent et relâchent les touches du clavier à vitesse relativement constante) ;

- l'antiplagiat ;

- la reconnaissance du style d'écriture : cet outil fonctionne, en parallèle de la reconnaissance de frappe au clavier, par l'analyse du contenu rédigé (régularité grammaticale, syntaxique...). 
12 Afin que le système puisse déterminer si l'étudiant est bien celui qu'il prétend être, il est nécessaire que ce dernier s'enregistre. Pour ce faire, il doit fournir au préalable un échantillon de ses données biométriques (un enregistrement vocal, par exemple) qui sera enregistré sur un serveur et qui servira de base pour établir la correspondance entre l'identité réelle de la personne et l'identité prétendue lors de la réalisation des activités. Le dispositif laisse la possibilité au corps enseignant d'activer ou de désactiver les instruments qu'il juge pertinents en fonction du contexte pédagogique.

Le principal objectif de TeSLA est l'authentification de l'étudiant à distance, autrement dit la possibilité de poser un diagnostic binaire (c'est lui, c'est elle devant l'écran, ou non) et ce en continu. Cette authentification est posée par les instruments via la production d'indices de ressemblance entre les échantillons envoyés lors de l'inscription de l'apprenant et les échantillons envoyés pendant l'accomplissement de la tâche d'évaluation. Des avertissements sont envoyés à l'enseignant si certains indices sont inférieurs à un seuil de sécurité prédéfini par les concepteurs des instruments. Les indices sont présentés sous la forme d'un tableau chronologique relatant les différentes données recueillies par les instruments biométriques au cours de chaque activité.

14 Ainsi, les enseignants peuvent avoir davantage de preuves que le travail produit est bien celui de l'étudiant officiellement inscrit. TeSLA se place dans une optique de vérification individuelle : il faut que le contenu évalué envoyé via la plate-forme en ligne soit rédigé des mains de l'étudiant inscrit et provienne de sa réflexion propre.

15 Explicitement ou implicitement, une série de comportements est attendue de la part d'un apprenant consentant à utiliser TeSLA (la notion de consentement est souvent utilisée dans le projet du fait des données à caractère privé qui transitent par le système pour l'usage des outils biométriques).

16 Lors de la démarche d'inscription, il s'agit de produire un contenu au cours d'une évaluation " de a à $\mathrm{z}$ ». Par cela, nous entendons « du cerveau aux doigts qui écrivent sur le clavier ». TeSLA vise à recréer à distance des conditions d'un examen écrit passé en salle, où, durant un temps imparti, l'apprenant n'a pas le droit de communiquer avec qui que ce soit et doit adopter un comportement non ambigu. Il est cependant évident que le système TeSLA, tel qu'il est pensé actuellement et utilisé seul, ne couvre pas toute l'étendue des comportements potentiels communément non admis en salle d'examen (le fait de parler n'est généralement pas pris en compte puisque l'instrument de reconnaissance vocale ne fonctionne pas obligatoirement, de même il n'y a aucun moyen de vérifier ce qui se trouve hors du champ de vision de la webcam...).

17 Certains comportements attendus sont aussi des situations engendrées ou potentiellement engendrées de fait par l'usage de TeSLA, bien qu'ils n'aient pas été pris en compte lors de la conception du projet. Il s'agit principalement de prérequis logistiques induits par l'utilisation de l'évaluation :

- un environnement permettant l'usage des instruments: aucune autre personne dans le champ de la webcam, pas de bruit parasite pendant l'usage de la reconnaissance vocale... Bref, un environnement que l'on imagine clos et calme ;

- la possibilité de libérer un espace de temps continu sans être dérangé : à nouveau, cela implique un environnement propice à la solitude. Quid si la personne disparaît du champ de vision de la webcam pendant un certain temps ? Le professeur en sera informé ;

- la connexion à internet doit avoir une qualité suffisante. Dans le cas contraire, le fonctionnement des instruments peut être gêné (cela s'applique surtout à la reconnaissance 
faciale et la reconnaissance de frappe au clavier, qui fonctionnent de manière continue pendant la durée de l'activité). enseignée et testée. Il n'agit que comme un moyen de vérifier l'identité de celui qui subit une évaluation pour la rendre valide. TeSLA est vendu comme " pédagogiquement neutre ", autrement dit, il laisserait aux enseignants l'autonomie d'utiliser comme ils le souhaitent cette boîte à outils mise à leur disposition.

\section{Processus d'appropriation par les utilisateurs}

Nos observations faites sur le terrain ont permis de mettre en avant trois éléments qui ressortent fortement des entretiens et qui témoignent de la recomposition de la relation pédagogique à laquelle participe TeSLA. L'usage d'une méthodologie aussi ouverte et exploratoire que la nôtre permet de rendre compte des pratiques des utilisateurs et de la façon dont ils y donnent sens.

\section{La surveillance via le miroir sans tain}

De nos observations sur le terrain ressort une forme d'opacification de la relation entre professeurs et étudiants. Le système TeSLA rend la surveillance exercée par le professeur peu visible pour l'étudiant. C'est la métaphore du miroir sans tain, dispositif utilisé notamment dans les commissariats de police afin d'observer des suspects placés dans une pièce sans que ceux-ci puissent voir concrètement la surveillance qui pèse sur eux. Dans ces circonstances, le suspect sait qu'il y a un dispositif en face de lui permettant de le surveiller mais il ne sait pas quelles en sont les modalités exactes (que surveille-t-on exactement? Surveille-t-on son langage extracorporel ? Le surveille-t-on constamment ? Combien de personnes se trouvent-elles derrière la vitre teintée ? Y a-til réellement quelqu'un qui surveille?). Le surveillé a donc conscience d'être potentiellement observé, mais sans être capable de décrire les informations exactes que le surveillant prélève à cet égard.

21 Concernant TeSLA, le caractère opaque de cette observation se situe à deux niveaux différents, s'additionnant l'un l'autre :

- le niveau algorithmique : il s'agit du prélèvement des données par les outils biométriques et de leur traitement. Les étudiants, et généralement les professeurs, ne connaissent pas le fonctionnement exact de ces équipements et sont donc incapables de rendre compte des critères qui sont pertinents selon le système. Seules des instructions pour permettre la collecte efficace de ces données sont communiquées ;

- le niveau interprétatif: l'usage que les professeurs font des tableaux des données biométriques recueillies est incertain. En effet, il est prévu par les concepteurs du projet que les professeurs soient libres de travailler sur ces données de la manière qu'ils jugent être la plus pertinente. Il n'y a donc pas de standardisation des interprétations de ces données, excepté le rapport entre chacune d'elles et un seuil de confiance prédéterminé par les concepteurs. En matière de lutte contre la triche, les liens entre le règlement intérieur des universités et l'intégration en son sein de TeSLA restent également obscurs pour les professeurs. 
Cette double dimension de flou entourant la surveillance des étudiants via TeSLA contribue à instaurer de la part des professeurs un regard différent de celui existant dans des institutions classiques (par exemple dans une salle d'examen). Ici, il s'agit pour les étudiants de «montrer patte blanche » compte tenu de la méconnaissance des facteurs influençant l'appréciation du professeur et la manière dont celui-ci utilise le système. "Montrer patte blanche » signifiant montrer à tout moment qu'aucune influence externe ne vient perturber le juste déroulement du processus d'évaluation.

\section{Approche comportementaliste}

L'observation à distance des étudiants régulée par le système TeSLA n'est pas étrangère aux principes pédagogiques originels de la formation en ligne. Andrew Feenberg et Edward Hamilton ${ }^{8}$ rendent compte de l'évolution des paradigmes de la formation en ligne en commençant par l'inspiration comportementaliste qui constitue la base des premiers écrits en la matière. Son principe est qu'avec l'éducation à distance il est impossible pour un professeur d'observer un étudiant de la même manière que s'il était physiquement présent. Du coup, le professeur, sur la base «du peu d'informations » qu'il peut obtenir à distance, essaye de déduire comment se déroule le processus d'apprentissage du côté de l'étudiant. Cela se limite fatalement à l'observation de comportements (rapidité à réaliser une activité, temps passé sur une même page...). Bien évidemment, il existe actuellement la possibilité de dialoguer avec l'étudiant, mais cela ne figure pas dans les caractéristiques de TeSLA, qui est uniquement un système "d'observation de comportements ${ }^{9}$ ». Rappelons que ce qui compte pour TeSLA concerne des comportements enregistrables (frappe au clavier, présence physique devant l'écran de l'ordinateur...).

Comme nous avons pu le voir lors de notre description du système, TeSLA sert à aider le professeur à émettre un jugement sur des étudiants. Conscient de ce fait, l'étudiant observé par TeSLA se retrouve du même coup conditionné à agir d'une certaine manière, implicitement requise. Concrètement, cela se révèle par des attitudes, des façons d'agir, parmi lesquelles le fait de ne pas oser bouger de certaines manières devant son ordinateur, de ne pas oser regarder dans certaines directions, car cela pourrait selon les dires de certains étudiants être interprété par le système comme du recopiage d'une autre source (alors que les instruments de biométrie présents ne font que de l'authentification et sont incapables d'interpréter des mouvements).

Autre façon d'agir: des connexions fréquentes à la plate-forme. En effet, TeSLA vise avant tout à être implémenté dans le cadre d'une évaluation en continu, où les étudiants réalisent des activités tout au long d'une période. Les professeurs eux-mêmes expliquent qu'ils désirent exploiter TeSLA dans un cadre continu afin d'obtenir davantage de suivi et de rendre plus difficile la triche (tromper le système de multiples fois étant plus difficile qu'une seule fois). Les étudiants sont donc "physiquement obligés » de se connecter à la plate-forme.

Dernière attitude relevée : l'utilisation des données recueillies par TeSLA au-delà du simple objectif d'authentification: certains professeurs imaginent aller plus loin et utiliser les tableaux de résultats dans d'autres cadres d'étude, notamment à des fins analytiques. Par exemple, les données recueillies sur la reconnaissance de frappe au clavier serviraient à voir à quel moment de l'examen l'étudiant est plus ou moins actif. Il s'agirait de fait d'un détournement de l'usage initialement prévu du système. 
Comme indiqué précédemment, TeSLA fournit uniquement, et ce, quel que soit l'instrument, des données quantitatives (des chiffres organisés chronologiquement qui s'affichent en vert, en jaune ou en rouge en fonction de leur valeur par rapport à un indice de confiance). La croyance en une science de la "quantification» des comportements humains est à rapprocher du comportementalisme des origines de la formation en ligne. TeSLA suppose la possibilité de calculer la conformité des étudiants à un comportement attendu de leur part (or le conditionnement évoqué plus haut est en partie provoqué par des croyances erronées et exagérées qu'ont les étudiants par rapport aux capacités du système).

\section{La décontextualisation}

La démonstration de l'aspect purement quantitatif des données fournies par TeSLA nous permet de rebondir sur sa dimension décontextualisante. Par cela, nous signifions le fait que ces chiffres ne sont aucunement sensibles au contexte d'usage dans lequel ils sont produits. Ceci vaut tant pour le contexte dans le sens restreint du terme (l'interaction entre l'étudiant et le système, son environnement direct d'usage : est-il ou est-elle chez lui ou chez elle? Dans une pièce fermée ou non? Bruyante ou non ? Ou avec la présence de tierces personnes à proximité ?) que dans le sens très large et macrosocial qui influe fortement sur son aisance avec le système (est-ce que la personne vit dans un milieu où les nouvelles technologies de communication sont présentes? A-t-elle un environnement socio-économique propice à la bonne compréhension du système ?).

Tous ces facteurs, sans détailler les cas particuliers, influent sur la manière dont les étudiants utilisent le système, et donc sur les chiffres produits par celui-ci. Le cas précis suivant, recueilli lors des entretiens, témoigne de la discrimination directe que les instruments biométriques de TeSLA peuvent potentiellement faire peser sur les étudiants : une dame peu habituée à utiliser un ordinateur devait réaliser des activités en se faisant authentifier par les outils de reconnaissance faciale et de frappe au clavier ${ }^{10}$. L'expérience révéla que l'outil de reconnaissance de frappe au clavier était très peu précis avec les personnes qui n'ont pas l'habitude de frapper au clavier. En effet, l'algorithme de cet outil suppose une forme de régularité dans la manière de presser et de relâcher les touches (la temporalité entre les frappes) et celle-ci n'est présente que chez les individus ayant acquis une forme de mémoire musculaire du geste de frappe. Les variations de temporalité étant trop fortes chez cette personne, l'algorithme renvoya des chiffres très bas, qui étaient censés trahir une volonté de tromper le système.

30 Une telle décontextualisation inclut avec elle certaines représentations sur la manière dont le professeur est capable de surveiller ses étudiants via TeSLA. Son regard ne peut se porter sur leur environnement social ou encore sur leurs aptitudes à utiliser les outils de formation en ligne, mais uniquement sur le fait que l'information rentrée à l'aide du clavier émane bien de la bonne personne.

\section{Un mérite individualisé ?}

31 Comme nous l'avons dit en introduction, TeSLA vise à apporter davantage de rigueur et donc de légitimité à l'évaluation en ligne. Les trois aspects découlant des entretiens que 
nous avons menés et qui ont été exposés ci-dessus mettent en évidence la particularité avec laquelle TeSLA tente de remplir cette tâche. Il s'agit de mettre en place une manière perçue comme égalitaire et objective de juger si l'acquisition du savoir par l'apprenant est satisfaisante.

Comme nous l'avons brièvement abordé dans notre point méthodologique, un tel objet technique, de par les comportements qu'il permet, ou pas, à certains acteurs, induit une norme et de ce fait une représentation du monde. Au-delà de l'instauration d'une nouvelle logistique pour juger les élèves, TeSLA tente de mettre en place de nouvelles normes d'égalité, et donc des présupposés spécifiques dans la manière de juger l'acquisition du savoir. Il s'agit d'une nécessité, comme nous l'avons expliqué dans l'introduction, du fait du contexte particulier dans lequel se situent ces universités ouvertes, visant à proposer un enseignement proche de celui des universités classiques malgré des contraintes de distance.

Il est bien évidemment délicat de tracer une limite entre les normes d'éducation incluses dans un cursus sans TeSLA et dans un cursus avec TeSLA, vu que le système sert à sécuriser des cours universitaires déjà existants et qu'il peut être implémenté avec une certaine liberté par le corps enseignant (rien ne dit pour l'instant que TeSLA doive être utilisé à une certaine fréquence, ou pour tel ou tel type d'activité, ce qui laisse ouverte une grande gamme d'interprétations par les usagers du corps enseignant). Néanmoins, par l'analyse, rapide dans le cadre de cette contribution, des entretiens passés auprès des concepteurs et des utilisateurs, nous pouvons mettre en évidence un certain déplacement dans les soubassements normatifs qui animent l'objet. Il était important de regarder les principes d'usage tels que pensés par les concepteurs de TeSLA et comment ceux-ci sont perçus et réinterprétés par les acteurs sur le terrain car les normes d'un tel objet technique ne sont pas gravées dans le marbre lorsque les concepteurs établissent des plans, mais proviennent plutôt de cet aller-retour entre objet et utilisateurs ${ }^{11}$.

\section{Les corps comme garants de l'objectivité}

Sur la base des trois grandes observations de terrain que nous avons mises en évidence (la surveillance par miroir sans tain, l'approche comportementaliste et la décontextualisation de l'apprentissage) se dégage une volonté d'objectivité pure repoussant toutes les contingences qui seraient dues à ce contexte particulier d'asymétrie temporelle et spatiale entre l'étudiant et le professeur. Créer de l'indubitable dans de telles conditions nécessite de se raccrocher à l'observation de traits extrêmement fondamentaux, dont les outils technologiques sont (théoriquement) capables de rendre compte sans altération. Ce dénominateur commun, postulat implicite sur lequel TeSLA se fonde afin de rendre ces diversités d'étudiants et de contextes d'usage comparables, est celui de l'objectivité des corps physiques.

Le fait de choisir délibérément ce critère comme garant de l'égalité et de la rigueur dans le jugement des apprenants est loin d'être anodin. Le système vise à neutraliser tous les aspects subjectifs pouvant entraver le jugement effectué par les enseignants. Pour cela, il permet d'observer ce que le corps a de plus calculable et descriptible par des variables quantitatives et ce à travers un voile d'opacité qui espère contribuer à assurer l'objectivité de l'observation. 
Caractériser de la sorte des normes et en déduire un principe fondateur qui assure la cohérence du système peut nous aider à interroger la transmission du savoir qui a lieu dans ce cadre. En nous posant la question "Qu'est-ce que la transmission du savoir via un dispositif tirant sa légitimité de l'observation objective des corps des apprenants?", nous sommes en mesure de donner un éclairage sur le profil de l'étudiant méritant qui émane de TeSLA.

\section{Individualité du mérite}

Du fait de cette authentification des étudiants sur la base d'une vérification physique de leur présence face à un écran d'ordinateur, TeSLA implique de manière assez directe une forme d'individualisation $d u$ processus d'apprentissage. Les trois tendances remontant du périmètre de l'enquête en témoignent: les outils biométriques sont conçus pour la surveillance d'une seule personne à la fois, ils engendrent potentiellement une forme d'analyse comportementale des étudiants et ils excluent du point de vue des professeurs toute donnée contextuelle.

TeSLA par exemple n'est pas équipé pour surveiller des étudiants effectuant un travail de groupe, il se concentre sur la vérification de la présence effective d'un apprenant devant son écran d'ordinateur pendant que celui-ci rédige son activité d'évaluation.

Le fait que TeSLA tende à exclure toute possibilité d'évaluation collective provoque d'ailleurs des réactions circonspectes chez certains professeurs. Une fois le candidat isolé de son contexte social et le savoir équitablement diffusé, comment expliquer les inégalités subsistantes en matière de résultats aux évaluations? Une certaine vision du mérite scolaire découle de ce regard ultra-individualisé sur l'étudiant. Puisque le mérite devient un ensemble de chiffres individuels et que tous les déterminants sociaux sont éclipsés, les causes imputées à ce mérite paraissent dès lors émaner de l'individu. Le mérite scolaire semble avoir été distribué au sein des étudiants sous la forme d'un capital spécifique, une forme de motivation (pour reprendre un terme couramment employé par les concepteurs du projet) permettant à l'apprenant d'utiliser au mieux des ressources (les matériaux de cours) dont il dispose équitablement. La focalisation sur les comportements objectifs des apprenants vise dès lors à rendre compte au mieux de ce mérite. En d'autres termes, en ne prenant en compte que ces aspects objectivables par la biométrie et la diffusion uniforme du savoir, on facilite une définition objective des mérites à promouvoir et donc une hiérarchisation des étudiants en fonction de cette définition.

Cependant, comme nous l'avons brièvement rappelé, une application technique influence le comportement de ses utilisateurs mais n'exerce pas de purs déterminismes. Notre travail de terrain ne nous permet pas d'affirmer qu'un professeur utilisant TeSLA verra toujours ses étudiants sous l'angle individualisé que nous avons tenté d'évoquer. Mais il est clair en revanche que, par le dispositif entonnoir que constitue le système en matière de surveillance des étudiants (une agrégation des comportements des étudiants sous la forme de chiffres) ainsi que par l'appropriation qui en a été faite par une partie des professeurs participant aux essais (que dans certains cas nous pourrions qualifier de dérives car non prévues par le système, comme le calcul de l'activité de l'étudiant alors que les données recueillies ne doivent normalement servir qu'à l'authentification), TeSLA implique malgré tout la représentation d'étudiants d'après des capacités individuelles prédéterminées. 


\section{BIBLIOGRAPHIE}

AKRICH Madeleine, «Les utilisateurs : acteurs de l'innovation », dans Akrich Madeleine, Callon Michel, Latour Bruno (dir.), Sociologie de la traduction : textes fondateurs, Paris, Presses des Mines, 2006, p. 253-265.

GROOTAERS Dominique, «Les mutations de l'égalité des chances à l'école », Courrier hebdomadaire du CRISP, $\mathrm{n}^{\circ} 1893,2005$, p. 5-43.

HAMILTON Edward, FEENBERG Andrew, «The technical codes of online education ", E-learning and

digital media, vol. II, $\mathrm{n}^{\circ} 2,2005$, p. 104-121.

INTRONA Lucas, « Disclosive ethics and information technology : disclosing facial recognition

systems ", Ethics and information technology, vol. VII, $\mathrm{n}^{\circ}$ 2, 2005, p. 75-86.

KAUFMANN Jean-Claude, L'entretien compréhensif, Paris, Armand Colin, 2016.

LEJEUNE Christophe, « Montrer, calculer, explorer, analyser : ce que l'informatique fait (faire) à

l'analyse qualitative ", Recherches qualitatives, vol. IX, 2010, p. 15-32.

SUCHMAN Lucy, Plans and situated actions : the problem of human-machine communication, Cambridge, Cambridge University Press, 1987.

\section{NOTES}

1. D. Grootaers, « Les mutations de l'égalité des chances à l'école ».

2. L. Introna, «Disclosive ethics and information technology: disclosing facial recognition systems ».

3. L. Suchman, Plans and situated actions: the problem of human-machine communication.

4. Ces entretiens furent étalés entre le septième et le douzième mois du projet (qui a duré trois ans) auprès de sept ingénieurs (avec deux grands types de profils : des ingénieurs travaillant sur les instruments de biométrie et ceux travaillant sur l'intégration de ces outils avec les plates-formes d'enseignement en ligne).

5. Ces entretiens furent étalés entre le vingt-cinquième et le trentième mois du projet. L'échantillon se constituait de six professeurs enseignant dans des universités ouvertes et de quinze étudiants de ces mêmes universités, tous ayant testé TeSLA.

6. C. Lejeune, «Montrer, calculer, explorer, analyser : ce que l'informatique fait (faire) à l'analyse qualitative ».

7. J.-C. Kaufmann, L'entretien compréhensif.

8. E. Hamilton, A. Feenberg, "The technical codes of online education ».

9. Au sens strict, le seul comportement dont TeSLA est censé rendre compte est la présence de l'étudiant en face de l'écran. Mais, sur la base de cela, les professeurs tendent à inférer davantage d'informations : motivation, manière de travailler...

10. Lors des essais du projet TeSLA, le système a été implémenté dans diverses universités européennes, certaines utilisant l'enseignement à distance pour toucher un public plus défavorisé. La prise en considération des aspects contextuels macrosociaux prend tout son sens ici. 
11. M. Akrich, « Les utilisateurs : acteurs de l'innovation ».

\section{RÉSUMÉS}

Dans le contexte de l'émergence des universités ouvertes qui proposent des cursus universitaires à réaliser entièrement en ligne, la mise au point d'une solution fiable et légitime pour attester la validité des évaluations est un enjeu crucial. Le projet TeSLA tente de répondre à cette problématique par l'authentification des apprenants par le biais d'outils de reconnaissance biométrique. L'usage de ceux-ci induit un certain mode de surveillance opaque et une analyse comportementaliste des étudiants embarquant une certaine notion du mérite scolaire qui devient ultra-individualisé, voire inné.

\section{AUTEUR}

\section{NATHAN DE VOS}

Chercheur en sociologie, Centre de recherche information, droit et société (CRIDS, université de Namur) 


\title{
Les enjeux de la numérisation des cartes et photographies anciennes dans la transmission des savoirs
}

\author{
Daniel Pouyllau et François Bart
}

Nous remercions les personnes suivantes pour leurs réalisations et pour leur collaboration: Caroline Abéla, Shadia Kilouchi et Carlos Jenart (centre Regards, CNRS), Philippe Laymond (bibliothèque de géographie - cartothèque du département de Géographie de l'université Bordeaux-Montaigne), Maud Pichardie, Nicolas Samoyault, Marie-France Pouyllau, Caroline Lefranc, Françoise Comet et Stéphane Pouyllau.

Plusieurs organismes en France et dans le monde ont entrepris au cours des vingt-cinq dernières années la numérisation de leurs fonds de cartes anciennes, géographiques et historiques (cartes topographiques et cartes thématiques), de plans cadastraux, de photographies aériennes et d'images satellitaires afin de développer leur communication. Les travaux réalisés par le centre d'information scientifique et technique du CNRS Regards à Pessac et par le consortium «ImaGEO, Images des géographes - Cartes et photographies ", financés par la très grande infrastructure de recherche (TGIR) Huma-Num ${ }^{1}$, s'inscrivent dans ces actions et permettent de sauvegarder des documents anciens relativement fragiles et de faciliter les comparaisons avec des documents plus récents produits par les nouveaux outils de la géomatique et de la cartographie 2.0. La numérisation et la description détaillée par des métadonnées de ces documents facilitent aussi la diffusion de plusieurs fonds concernant des pays d'Afrique, d'Amérique et d'Asie, en particulier vers les chercheurs des pays couverts par ces cartes ; le problème principal de la diffusion vers les pays des Suds est lié aux contraintes des accès à internet dans ces pays, parfois aux limites de la distribution de l'électricité et au manque de matériel adapté à la lecture de cartes de grand format, les lecteurs utilisant souvent leur téléphone portable pour la consultation. D'autres fonds de photographies scientifiques, en noir et blanc et en couleurs, en particulier de diapositives prises par des chercheurs depuis les années 1950, commencent aussi à être numérisés. 
2 La diffusion de ces ressources est effectuée via l'archive ouverte MédiHAL diffusée par le Centre pour la communication scientifique directe (CCSD-CNRS) ${ }^{2}$. Grâce à une indexation détaillée et aux annotations apportées par les auteurs ou par des documentalistes-iconographes en collaboration avec des chercheurs, cette archive facilite la transmission des savoirs géographiques. La participation à des formes d'indexation collaborative est recherchée pour compléter et améliorer la description scientifique de ces supports.

\section{Les fonds de cartes et de photographies anciennes à Bordeaux}

3 L'histoire des relations entre Bordeaux et l'Afrique et les Antilles a donné lieu, au cours des derniers siècles, à de nombreuses publications sur les pays africains, en particulier francophones, et sur les Antilles françaises. Plusieurs organismes publics et privés (assemblées délibérantes, universités, établissements consulaires, associations, entreprises) ont développé à Bordeaux des fonds documentaires, des bibliothèques, des services d'archives et des centres de documentation qui ont facilité l'organisation, la conservation et la diffusion de ces documents.

\section{La Société de géographie commerciale de Bordeaux}

4 Créée en 1874 par un groupe de négociants, d'armateurs et de professeurs soutenu par la chambre de commerce de Bordeaux, elle a été la troisième société de géographie créée en France après celles de Paris (1821) et de Lyon (1873), et la vingtième du monde. Reconnue d'utilité publique par décret du 31 août 1896, elle favorisait, à travers son bulletin et ses conférences, la diffusion de connaissances scientifiques sur les régions $\mathrm{du}$ sud-ouest aquitain et sur les pays d'outre-mer avec lesquels le port de Bordeaux entretenait des relations commerciales importantes. La Société de géographie de Bordeaux ${ }^{3}$ est l'héritière de la Société de géographie commerciale de Bordeaux : cette société savante organise chaque année des conférences, les Lundis de la géographie ${ }^{4}$.

5 Le fonds de la Société de géographie commerciale de Bordeaux et celui de l'ancien Institut colonial de Bordeaux ont été déposés à la bibliothèque pluridisciplinaire de l'université de Bordeaux depuis 1974. Ces fonds sont composés principalement de revues périodiques géographiques des $\mathrm{xIX}^{\mathrm{e}}$ et $\mathrm{xx}^{\mathrm{e}}$ siècles sur le thème de l'empire colonial français (dont les Annales de l'Institut colonial de Bordeaux, le Bulletin mensuel: renseignements commerciaux et économiques), ainsi que d'ouvrages anciens et d'atlas des $\mathrm{XVIII}^{\mathrm{e}}$ et $\mathrm{XIX}^{\mathrm{e}}$ siècles sur les thèmes de la découverte et du voyage (Afrique, Océanie), parmi lesquels la Description de l'Afrique par Jean Léon l'Africain, le Petit atlas maritime de Bellin, ouvrages possédés aussi par la BNF, mais il est intéressant de signaler leur présence à Bordeaux ${ }^{5}$.

6 Le fonds de cartes géographiques de la Société de géographie commerciale de Bordeaux a par contre été versé à la bibliothèque de géographie - cartothèque de l'université Bordeaux-Montaigne décrite ci-après et, pour certains pays d'Afrique, d'Amérique latine et d'Asie du Sud-Est, au fonds du centre Regards (fig. 1). 
Fig. 1. - Madagascar : carte géologique et minière.

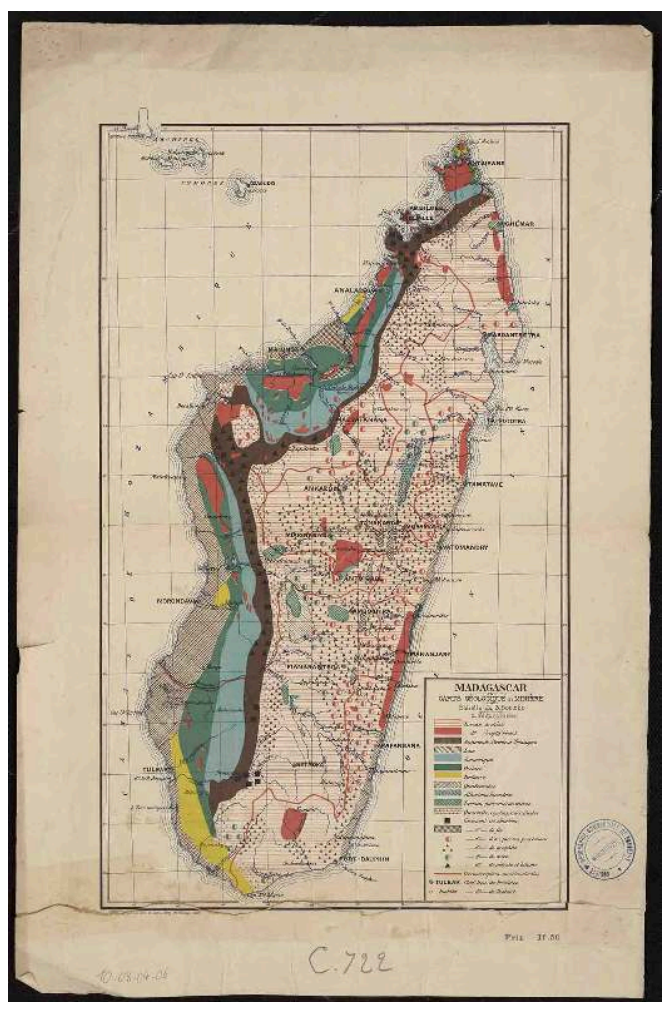

Source : http://1886.u-bordeaux-montaigne.fr/items/show/9816

CC - Public Domain Mark 1.0

\section{La bibliothèque de géographie - cartothèque de l'université Bordeaux-Montaigne}

7 Elle gère un fonds classique pour un département d'université (ouvrages, revues, photographies aériennes et images satellitaires, diapositives) mais elle possède aussi un fonds ancien très riche, constitué en particulier de 73000 cartes $^{6}$. Dans ce fonds, les cartes déposées par la Société de géographie commerciale de Bordeaux sont signalées dans le SUDOC (Système universitaire de documentation) et en cours de numérisation et de mise en ligne dans la bibliothèque numérique 1886 - Collections patrimoniales numérisées de Bordeaux-Montaigne ${ }^{7}$, et également accessibles sur Gallica ${ }^{8}$ (fig. 2). Les opérations de numérisation ont bénéficié de l'appui de la TGIR Huma-Num dans le cadre du consortium ImaGEO qui est présenté ci-dessus. 
Fig. 2. - Fouta Central : ligne télégraphique de Saldé à Matam.

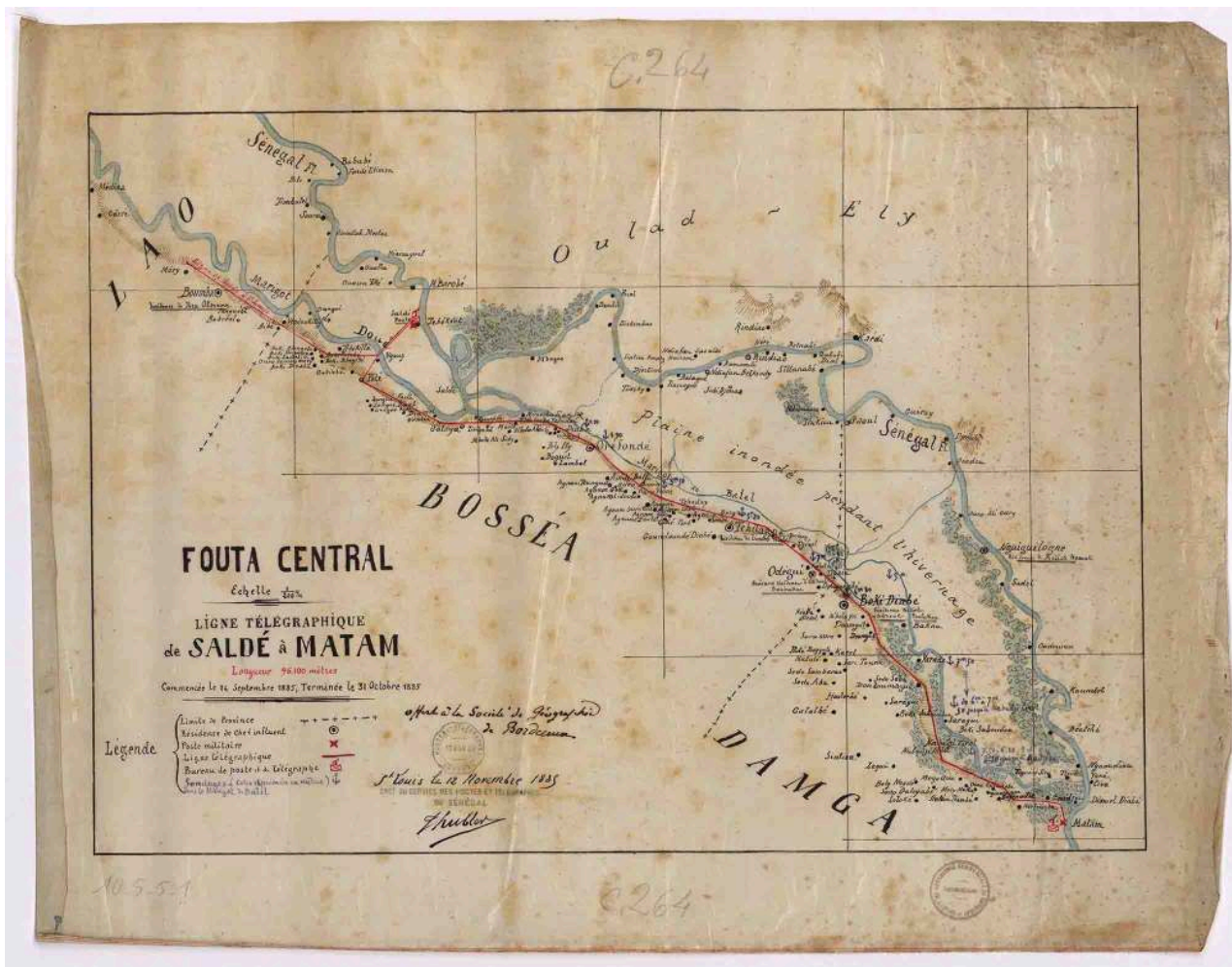

Source : http://1886.u-bordeaux-montaigne.fr/items/show/9344

CC - Public Domain Mark 1.0

\section{Le centre d'information scientifique et technique du CNRS Regards}

C'est la composante documentaire de Passages, unité mixte de recherche regroupant le CNRS, l'université Bordeaux-Montaigne, l'université de Bordeaux, l'université de Pau et des Pays de l'Adour et l'École nationale supérieure d'architecture et de paysage de Bordeaux (ENSAP). Créé en 1968 sous le nom de CEGET (Centre d'études de géographie tropicale, laboratoire propre du CNRS), il comprend une bibliothèque de recherche, une cartothèque-photothèque et un système de bases de données. Le fonds documentaire couvre les domaines suivants: géographie, aménagement, développement, environnement, santé et sociétés. Composé de 40000 monographies, de dizaines de milliers d'articles publiés dans plus de 1400 titres de revues, 15000 cartes, 22000 photographies aériennes et plus de 20000 diapositives prises sur le terrain par des chercheurs à partir des années 1950, ce fonds a fait l'objet dès 1972 d'un traitement à travers la base de données de Regards, qui signale plus de 167000 documents.

9 Le fonds de la cartothèque-photothèque de Regards est également en cours de numérisation : cette initiative concerne à ce jour l'Amérique et l'Afrique.

Pour l'Amérique :

- la Caraïbe et les îles des Antilles : ce fonds, composé de 1061 cartes et plans publiés de 1702 à 1988, est structuré en 29 collections couvrant pratiquement tous les pays de la zone. D'autres fonds sur cette région du monde ont été aussi numérisés et représentent des ressources importantes: les départements français Guadeloupe, Martinique et Guyane française mais aussi Haïti. Ces fonds de cartes sont liés à l'édition de quatre Atlas des 
départements français d'outre-mer publiés de 1975 à 1982 par le CEGET (Martinique, 119 cartes de l'Atlas publié en 1977 ; Guadeloupe, 122 cartes ou reproductions de cartes publiées de 1667 à 1982 [fig. 3] ; Guyane, 198 cartes ou reproductions publiées de 1599 à 1988) ;

Fig. 3. - Guadeloupe : carte géomorphologique.

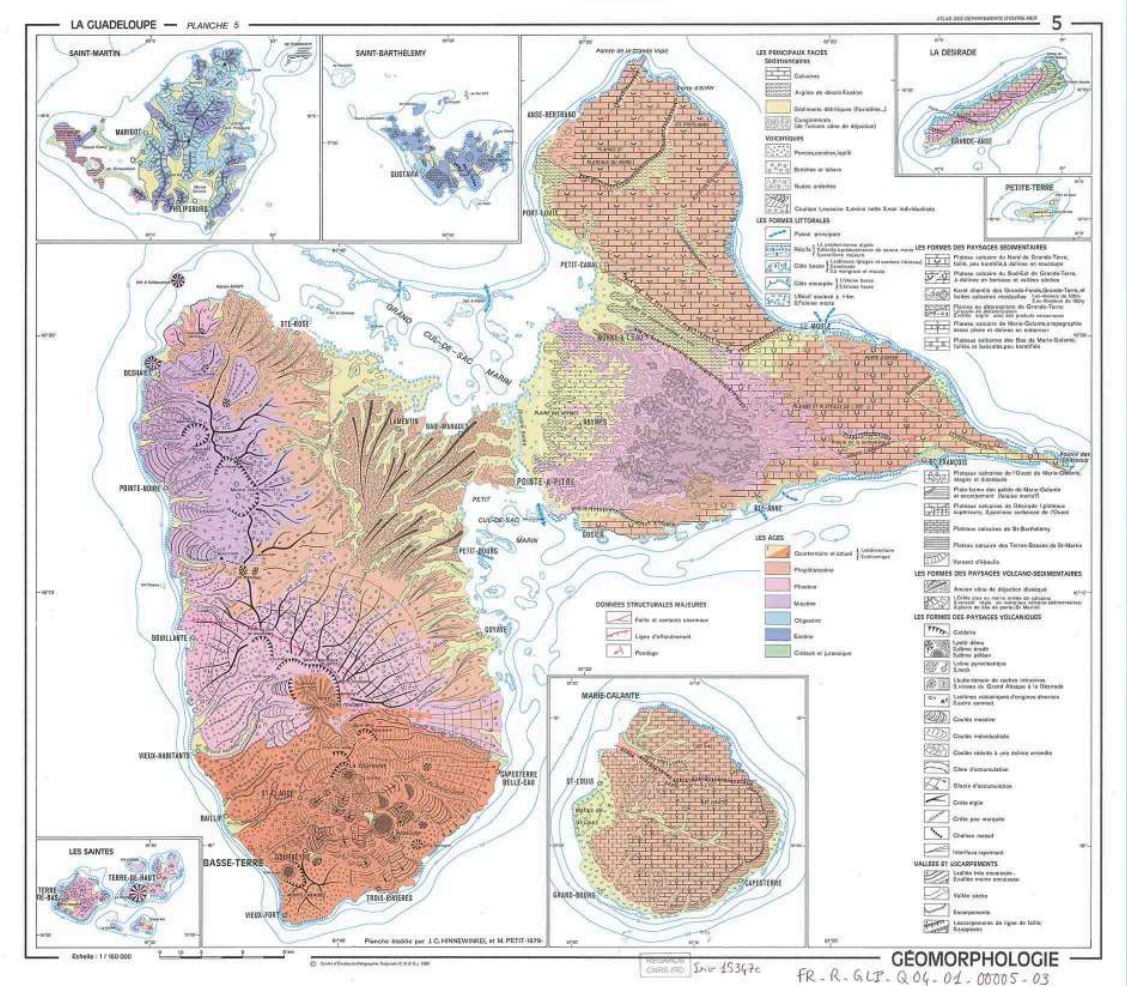

CC BY-NC-SA 4.0

- l'Argentine : 610 cartes et plans cadastraux composent ce fonds, légué en grande partie par le professeur Romain Gaignard (université de Toulouse - Le Mirail) qui a présenté en 1979 à Bordeaux une thèse remarquable sur la Pampa argentine de 1872 à 1979. Le fonds de cartes et de plans cadastraux-couvre en majorité les provinces de Buenos Aires et de Córdoba (fig. 4), et de façon plus restreinte les provinces de la Pampa et de Santa Fé ; 
Fig. 4. - Province de Córdoba, La Francia (Argentine) : plan cadastral.

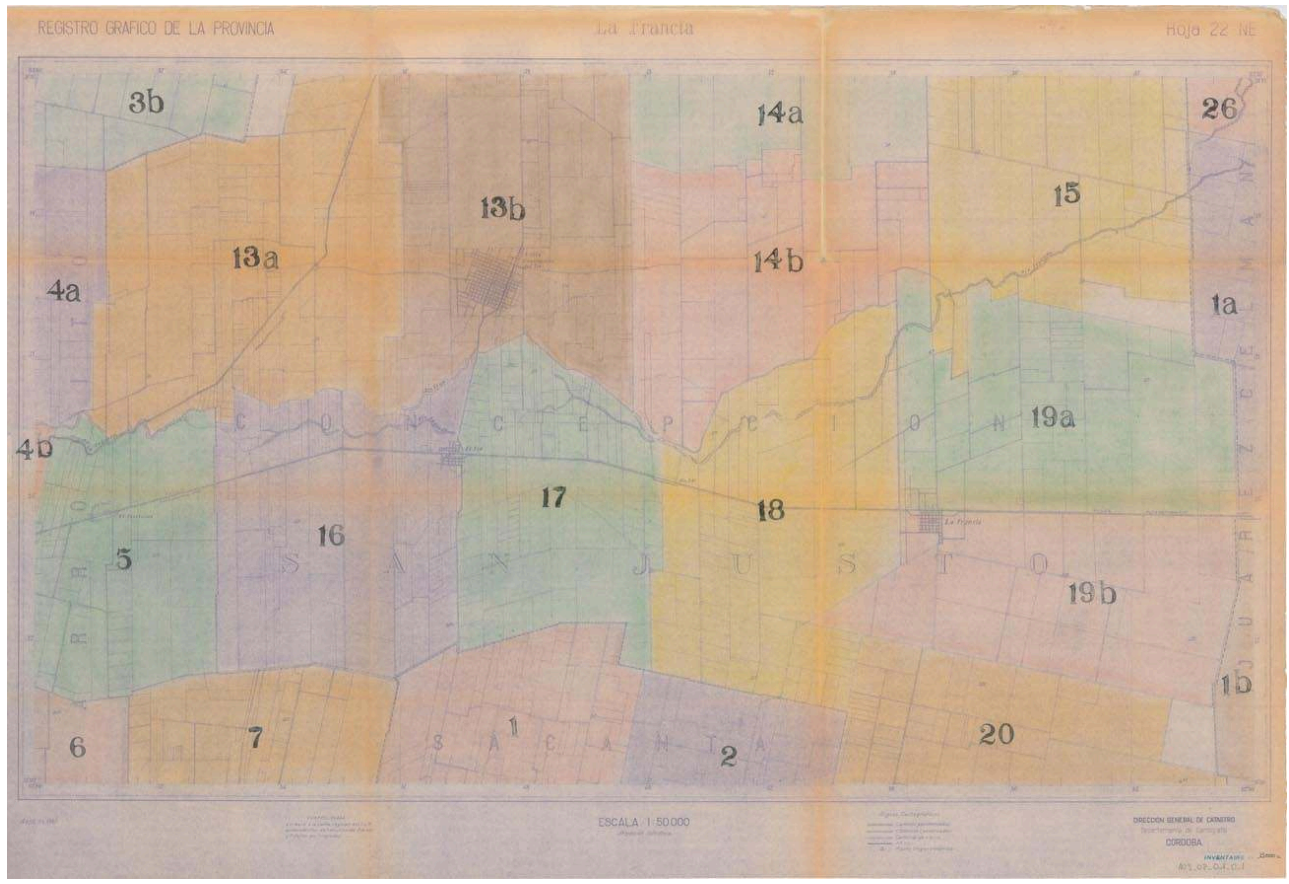

Échelle 1/50 000 (1967). Source : https://www.navigae.fr/resources/images/regards/argentine/ oai_nakala_fr_hdl_11280_b383bdc9.jpg

CC BY-NC-SA 4.0

- le Brésil : 478 cartes topographiques régulières à des échelles complémentaires, cartes thématiques du milieu naturel et de l'aménagement et images satellitaires et radar. Le fonds contient aussi un Atlas de 399 planches publié par le géographe Hervé Théry ;

- la Colombie : 130 cartes topographiques et thématiques publiées de 1881 à 1995 ;

- le Venezuela : 747 documents, probablement le fonds le plus important en France sur ce pays. Il couvre l'ensemble du territoire fédéral du Venezuela avec des cartes publiées de 1825 à 1991: cartes topographiques de reconnaissance à diverses échelles, cartes topographiques régulières à des échelles complémentaires $(1 / 5000$ à $1 / 6000000)$ et cartes thématiques (géologiques, géomorphologiques, pédologiques, hydrologiques, climatologiques, politiques, de la végétation et de l'utilisation des sols, économiques et industrielles, routières). Le fonds comprend de nombreuses images satellitaires Landsat et SPOT, des images radar à visée latérale SLAR Goodyear et des images SIR-A prises depuis la navette spatiale américaine. Ce fonds correspond aux travaux de chercheurs du centre ayant travaillé dans ce pays entre 1972 et 1982 (fig. 5). 
Fig. 5. - Guanare-Masparro (Venezuela) : carte géomorphologique.

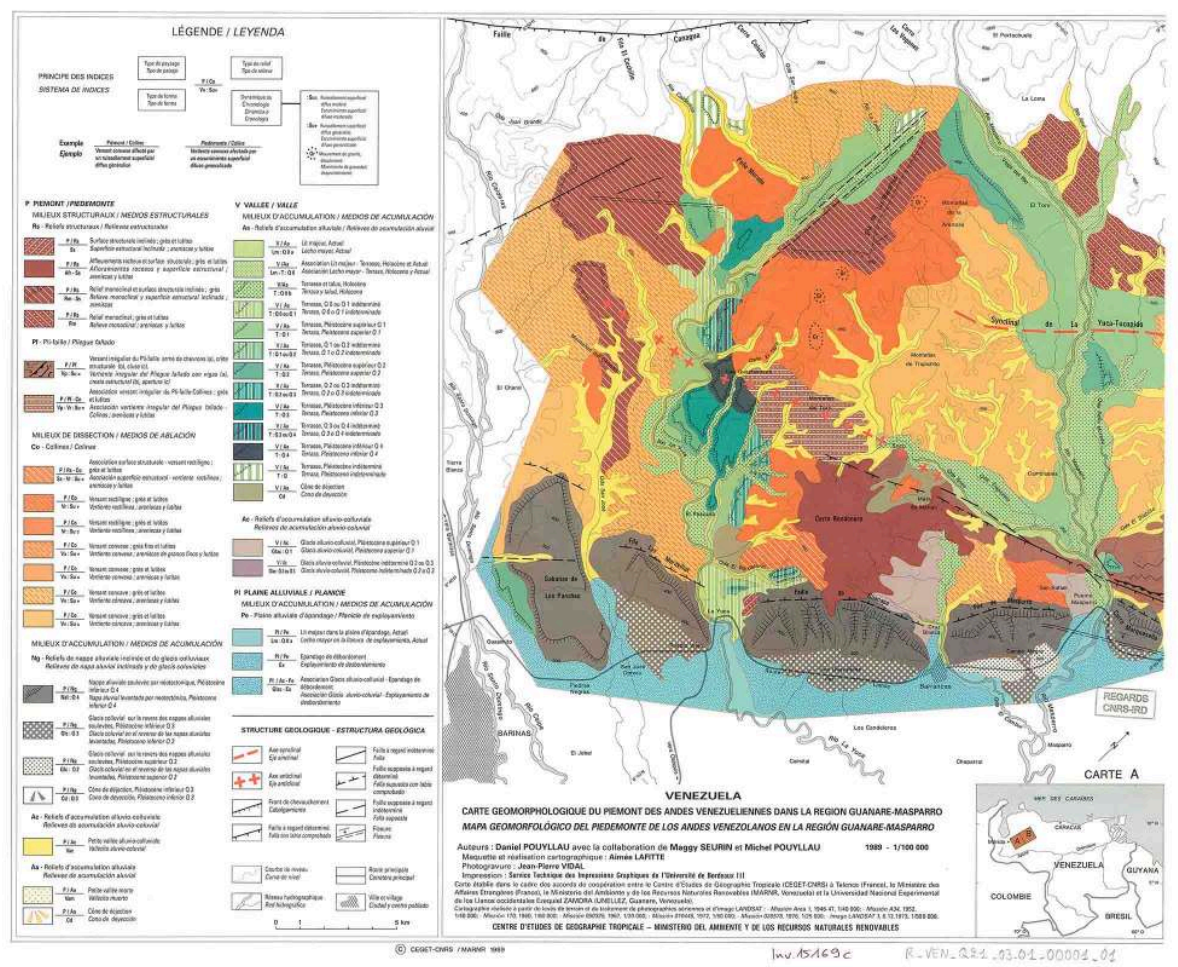

Échelle 1/100 000 (1989). Source : https://www.navigae.fr/map?

lang=fr\&ids=oai:nakala.fr:hdl_11280_d58ae330

CC BY-NC-SA 4.0

\section{Pour l'Afrique :}

- le Burundi : lors de la réalisation de l'atlas du Burundi publié en 1979 par le CEGET, plus de 200 cartes ont été rassemblées et éditées entre 1937 et 1979. Ce fonds a été complété par des cartes éditées jusqu'en 1986 ;

- Madagascar: ce fonds, l'un des plus importants de Regards, rassemble 1319 cartes correspondant aux débuts de la cartographie régulière de ce pays (fin du XIX ${ }^{\mathrm{e}}$ siècle et début $\mathrm{du} \mathrm{xx}^{\mathrm{e}}$ siècle), ainsi que les cartes topographiques de reconnaissance à diverses échelles, les cartes topographiques régulières à des échelles complémentaires $(1 / 20000$ à $1 / 200000)$ et les cartes thématiques (fig. 6). Ce fonds a été utilisé pour plusieurs recherches ${ }^{9}$ permettant de montrer le lien entre la carte comme outil descriptif de la nature et la représentation de situations environnementales successives dans l'histoire de ce pays ${ }^{10}$; 
Fig. 6. - Ambilobe (Madagascar) : carte géomorphologique.

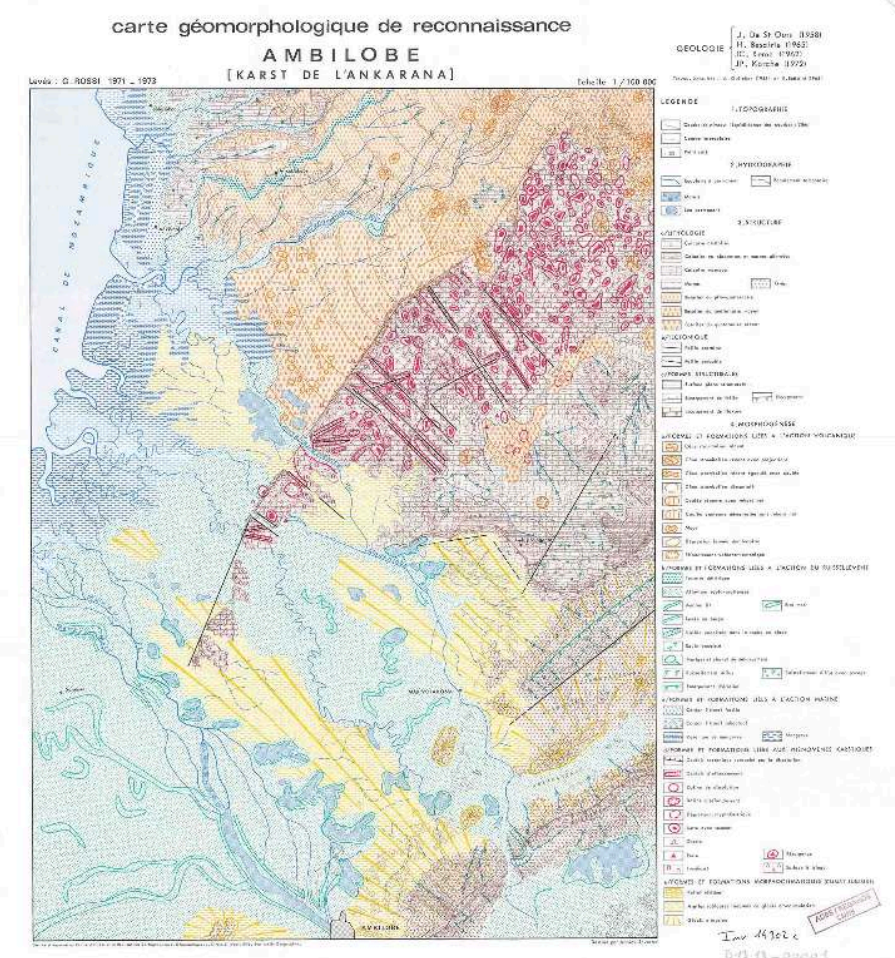

Georges Rossi (CNRS). Échelle 1/100 000 (1973). Source : https://www.navigae.fr/map? textSearch=Ambilobe\%20\&lang=fr\&ids=oai:nakala.fr:hdl_11280_cc7140e3

CC BY-NC-SA 4.0

- le Niger : 221 cartes, publiées entre 1882 et 1993, couvrant le Niger mais aussi les anciennes régions correspondant actuellement au Mali, à la république de Guinée et au Nigéria. Ce fonds a été numérisé dans le cadre du développement d'un partenariat avec l'université Abdou-Moumouni de Niamey.

Plusieurs autres fonds, principalement des pays d'Afrique et d'Asie, sont en cours de numérisation, l'ensemble des ressources de cette cartothèque s'élevant à plus de 15000 cartes et plans.

Le fonds de photographies scientifiques de Regards comprend plus de 35000 clichés, en noir et blanc et en couleurs, en particulier des diapositives prises sur le terrain par des chercheurs pour illustrer leurs recherches, de 1950 à 1980, ainsi que des photographies aériennes et d'images satellitaires. Le fonds de diapositives en couleurs commence à être numérisé : 4000 diapositives sont accessibles sur MédiHAL dans la Photothèque du centre d'information scientifique et technique Regards ${ }^{11}$, également avec le soutien de la TGIR Huma-Num. Les diapositives des pays suivants sont notamment accessibles pour l'Afrique (Burkina Faso, Burundi, département de La Réunion, Kenya, Madagascar, Ouganda, république de Guinée, Tanzanie); pour l'Amérique (Argentine [fig. 7], Brésil, Venezuela); pour l'Asie (Vietnam) ${ }^{12}$. 
Fig. 7. - Chaco (Argentine) : ouvriers agricoles.

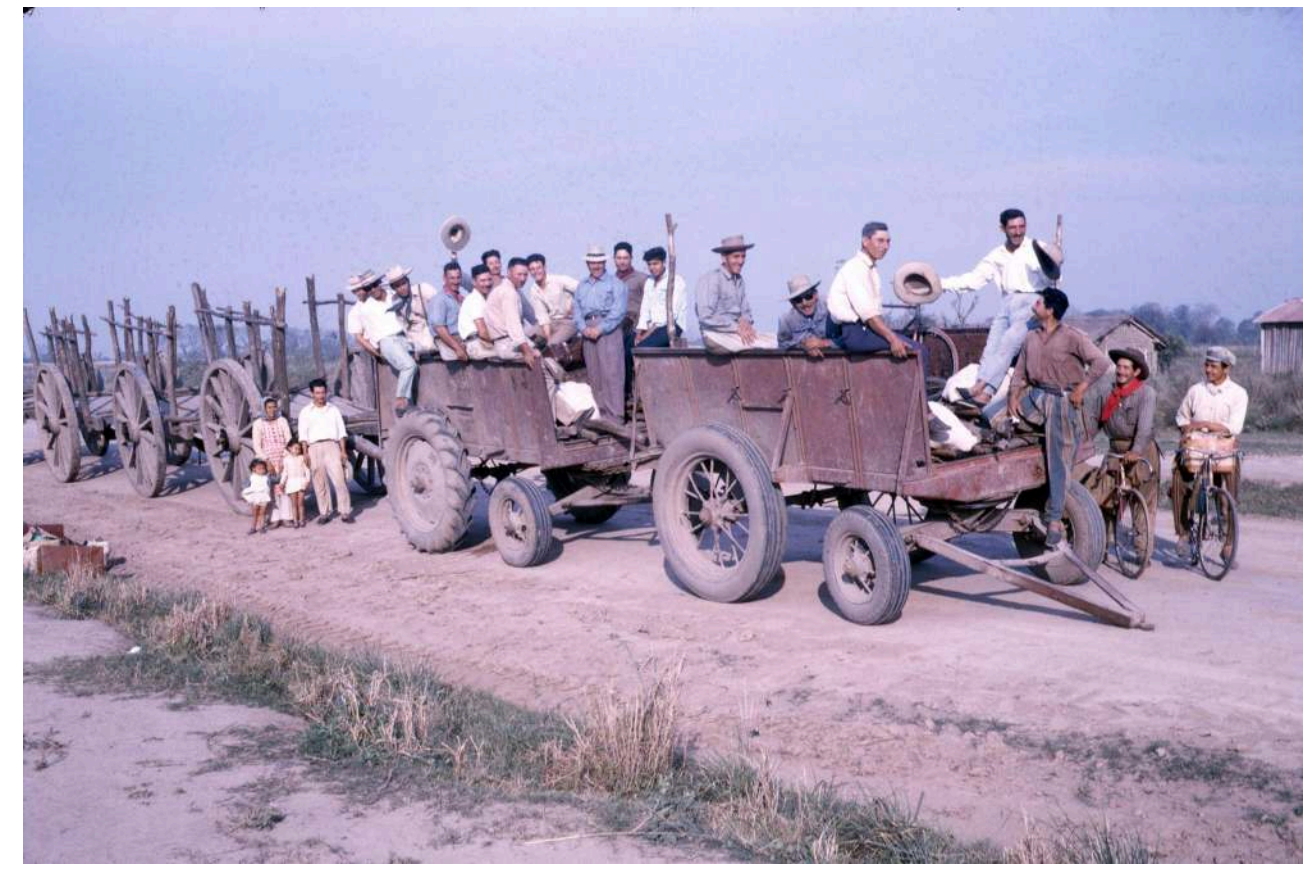

Cliché Romain Gaignard (1960). Source : https://medihal.archives-ouvertes.fr/ROMAINGAIGNARD/ medihal-00697464v1

CC BY-NC-SA 4.0

\section{Techniques et méthodes utilisées pour la numérisation et la description des cartes et des photographies}

Les fonds sont ordonnés en fonction d'un plan de classement générique type mais adapté à chaque pays, car les fonds ne sont pas équivalents et l'ensemble des types de cartes ne sont pas présents dans chacun d'eux. En général, le plan de classement suit l'ordre : cartes anciennes, cartes topographiques, cartes de géographie physique, cartes de géographie humaine et économique. À l'intérieur de chaque sous-ensemble, les cartes sont classées en fonction de l'échelle puis de la date d'édition ou d'impression.

L'inventaire des cartes du centre Regards est fait en suivant la norme de format de description EAD (encoded archival description) et rédigé conformément à la norme ISAD(G) (international standard archival description - general) v2 : chaque fonds est donc identifié et présenté selon la hiérarchie de collections, séries, sous-séries et pièces.

L'indexation permet de donner une description précise du contenu de chaque carte : en particulier les mots-matières et les noms géographiques, mais aussi les noms de personnes, des collectivités, des dates indiquées sur les cartes. L'indexation thématique est faite en utilisant le thesaurus thématique " géographie-développement » du centre Regards avec une possibilité de complément par des mots libres ${ }^{13}$.

La géolocalisation de chaque carte est faite en utilisant le système Google Maps : cela permet de proposer à la fois la reproduction de la carte numérisée et l'extrait des images satellites de Google avec la possibilité de zoomer à la fois sur l'image de la carte et sur les images de Google Maps. L'utilisation des API ${ }^{14}$ de Google Maps permet ainsi 
d'enrichir l'information proposée à l'utilisateur de ces fonds numérisés. Par contre, le Géoportail de l'IGN n'est pas utilisé car il ne couvre pas ces fonds de nombreuses régions du monde.

18 La numérisation des cartes est faite en général au format TIFF avec une définition de $300 \mathrm{DPI}^{15}$. Dans certains cas, les supports ne permettent pas une bonne reproduction au format TIFF, par exemple pour les cartes tirées sur du papier diazoïque (ou tirages de plans), imprimées ou dessinées sur calque. La reproduction au format TIFF entraîne par ailleurs une extension de la coloration dans les zones de pliure, alors qu'elle est plus réduite au format JPEG. Dans les cas de livrets de commentaires associés à la carte (fréquents pour les cartes géologiques, pédologiques ou de la végétation), la numérisation du livret est faite au format PDF.

Pour en permettre l'interopérabilité ${ }^{16}$, les métadonnées des documents contenus dans les inventaires sont publiées sur la plate-forme Archive numérique d'objets et de matériaux iconographiques (AOMS) du Centre national pour la numérisation de sources visuelles (CN2SV). AOMS est moissonné par Isidore, plate-forme permettant la recherche et l'accès aux données numériques et numérisées des publications en sciences humaines et sociales développée par la TGIR Huma-Num.

L'accès aux fonds de cartes est réservé à des chercheurs dans le cadre de leurs travaux avec les limites classiques de réutilisation des données dans le domaine de la recherche scientifique : ne pas modifier les documents reproduits, faire mention du copyright ou $\mathrm{du}$ droit d'auteur relatif au document, faire tout ce qui est raisonnablement possible pour assurer la précision des documents reproduits et indiquer un lien pérenne vers la source. D'autre part, des négociations sont en cours avec plusieurs partenaires étrangers et avec l'IGN pour la diffusion de cartes sous droits, en particulier pour les publics de chercheurs des pays des Suds, notamment de pays africains; nous reviendrons sur cette situation dans notre troisième partie.

21 L'accès aux fonds de cartes du centre Regards est facilité par l'utilisation du progiciel libre Pleade, largement utilisé dans le domaine des archives en France, et disponible sur le serveur AOMS du CN2SV. Une évolution est en cours avec le développement d'un outil de recherche permettant de consulter ces données : Navigae, nouvelle plate-forme de recherche au service de la valorisation de données géographiques patrimoniales ${ }^{17}$.

Les cartes du fonds de la Société de géographie commerciale de Bordeaux versées dans le fonds de la bibliothèque de géographie - cartothèque de l'université BordeauxMontaigne sont quant à elles décrites dans Babord+, le catalogue du réseau des bibliothèques universitaires de Bordeaux, ainsi que dans le SUDOC, géré par la Direction de la documentation des deux universités de Bordeaux, l'université de Bordeaux et l'université Bordeaux Montaigne. Les fonds numérisés sont également accessibles sur Gallica.

23 Les diapositives du fonds Regards sont en général de marque Kodachrome 64 ou 25 et elles ont été conservées dans de très bonnes conditions, dans des tiroirs métalliques à l'abri de la lumière; les films Kodak Ektachrome, Agfachrome, Fujichrome et autres sont en général moins bien conservés. La numérisation est réalisée avec une résolution de 1800 ou 3600 DPI et les fichiers sont disponibles en deux formats : TIFF et JPEG ; la profondeur de la numérisation est de 16 bits pour le TIFF et de 8 bits pour le JPEG. photographies. L'indexation détaillée (mots-clés thématiques et géographiques) est 
faite selon les mêmes principes que ceux utilisés pour l'indexation des cartes. Des annotations ont été reproduites lorsque les auteurs les avaient indiquées et les documentalistes-iconographes rédigent régulièrement des compléments sous la forme d'un résumé. La localisation géographique est donnée pour chaque photographie par l'indication de la longitude et de la latitude de la prise de vue : cela permet l'utilisation des API de Google Maps et la génération de l'extrait de l'image satellitaire qui apparait en dessous de la reproduction de la diapositive. Un complément important est ainsi donné à l'information fournie par l'image en situant la photographie ancienne sur l'imagerie satellitaire de Google Maps avec, parfois, la possibilité de visualiser l'état actuel du lieu de la prise de vue, lorsque l'outil Street View est proposé par le système Google.

Les diapositives sont diffusées en général avec une licence Creative Commons 4.0, demandant aux utilisateurs de ces photographies d'en indiquer la paternité et de ne pas en faire d'utilisation commerciale ni de modification. Dans quelques cas, les auteurs ont demandé d'indiquer une limite de type copyright. Les utilisateurs de MédiHAL sont soumis aux règles habituelles du bon usage des données dans le monde scientifique : respect des travaux originaux, mention des auteurs originaux et du lieu de conservation, respect et accord des personnes photographiées. L'auteur transmet à MédiHAL, à titre non exclusif, le droit de diffusion de son œuvre dans les conditions de l'accès libre et gratuit. La propriété intellectuelle des documents déposés reste entièrement celle du ou des auteurs.

La description et l'indexation détaillées, les annotations apportées par les auteurs ou par des documentalistes-iconographes facilitent la transmission des savoirs géographiques. La mise en place d'une participation à des formes d'indexation collaborative est recherchée pour pouvoir compléter et améliorer la description scientifique de ces supports.

\section{La diffusion des cartes et photographies et la transmission des savoirs}

Deux orientations principales ont été prises pour la diffusion des cartes et, dans une moindre mesure, pour la diffusion des photographies diapositives, avec l'objectif de faciliter la transmission des savoirs :

- la diffusion vers les milieux de la recherche en France et dans les pays où l'accès à internet est généralisé et performant;

- la recherche de méthodes adaptées de diffusion vers les étudiants et les enseignants de pays d'Afrique au sud du Sahara et de Madagascar, pays où l'accès à l'électricité et à internet est loin d'être généralisé.

Les fonds de cartes ont été largement utilisés dans les milieux de la production de recherche en France dans les années 1960-1980, en particulier pour la production d'atlas régionaux ou nationaux. Plusieurs universités ont ainsi produit des atlas sur les régions françaises et des atlas nationaux en liaison avec les accords de coopération développés avec des pays ou des régions d'Afrique, d'Amérique latine, d'Asie et d'Océanie. Cela a été le cas à Bordeaux, à la fois à l'Institut de géographie (devenu département de Géographie de l'université Bordeaux-Montaigne) et au CEGET (les fonds documentaires de ce centre sont maintenant gérés par le centre Regards). 
Pour produire ces atlas, de nombreuses cartes ont été achetées ou demandées en prêt afin de compléter les travaux de terrain et de photo-interprétation : elles ont servi de base à certaines productions, l'objectif de ces atlas étant d'éditer un document scientifique de l'état de la région ou du pays à un moment donné. L'Institut de géographie de Bordeaux a ainsi produit l'atlas d'Aquitaine, plusieurs atlas de villes d'Aquitaine, l'atlas du Burundi en 1979 dans le cadre d'une association proche de l'université, l'atlas de Maurice en 1997 ; le CEGET a de son côté édité les quatre atlas des départements d'outre-mer (la Réunion en 1975 en coédition avec l'IGN, la Martinique en 1977 en coédition avec l'IGN, la Guyane en 1979 en coédition avec l'ORSTOM [IRD], et la Guadeloupe en 1982); l'atlas d'Haïti a lui aussi été publié par le CEGET en 1985. Les fonds de cartes utilisés pour la production de ces atlas et les planches d'atlas ellesmêmes ont été numérisés pour faciliter leur diffusion, comme tout document aujourd'hui accessible par internet.

31 Les statistiques d'utilisation des fonds de cartes numérisées par des chercheurs ont montré, pour le cas des cartes de Madagascar, une utilisation quatre fois plus importante au cours des quatre dernières années : ce fonds est en effet le premier qui a été numérisé et est accessible depuis 2009. Trois programmes de recherche ont été conduits dans trois régions de ce pays et le centre Regards a fourni des copies des cartes numérisées, en particulier pour des équipes de recherche malgaches, sous la forme de disques durs amovibles. Il faut préciser que Madagascar avait demandé, lors de son indépendance en 1960, la rétrocession de la propriété des cartes et photographies aériennes réalisées par les divers services géographiques français ayant opéré dans et sur le pays.

L'utilisation des cartes anciennes et la comparaison entre des séries de cartes sur plusieurs dizaines d'années ont permis par exemple, dans le cas de Madagascar, de donner de nouvelles interprétations sur l'évolution du milieu naturel et en particulier sur l'évolution des forêts : la même carte, plus ou moins modifiée, a été largement utilisée par de nombreux opérateurs donnant ainsi des conclusions très différentes.

L'accès à la documentation conservée en France est facile depuis les pays occidentaux et nettement plus complexe depuis certains pays d'Afrique où l'accès à internet est très limité, y compris dans les capitales, et où l'électricité n'est pas distribuée régulièrement. L'accès à l'information, lorsque c'est possible, s'effectue par exemple pour les étudiants par l'intermédiaire d'un téléphone portable, outil peu adapté à la recherche bibliographique dans des bases de données et encore moins adapté à la consultation de thèses ou de cartes, par exemple. Les tablettes sont encore peu diffusées et les salles de lecture des bibliothèques sont encore peu fournies en ordinateurs en libre accès. Il est fréquent par exemple de voir un seul ordinateur au bureau des bibliothécaires, situation que l'on connaissait en France il y a vingt-cinq à trente ans. Certains étudiants possèdent un ordinateur mais d'autres demandent à leurs condisciples ou aux enseignants de leur prêter le leur.

Deux orientations sont actuellement en cours de réflexion à Regards (CNRS) et à l'université Bordeaux-Montaigne. La première est la recherche des modalités de signature de conventions avec les organismes concernés pour leur donner des copies des fichiers des cartes numérisées afin qu'ils les diffusent sur des serveurs locaux pour faciliter l'accès à ces informations. Une procédure est en cours avec Madagascar mais elle n'a pu encore être mise en place. 
La deuxième est la mise en œuvre d'un système de diffusion à la demande de copies de documents vers les étudiants, en particulier dans le cadre de leurs travaux de recherche en deuxième et troisième cycles. L'association RAFID (Réseau d'acteurs pour la diffusion des fonds d'information et de documentation pour le développement ${ }^{18}$ ) s'est associée au centre Regards dans ce projet pour essayer de développer ces procédures avec l'université Abdou-Moumouni de Niamey (Niger). Les demandes des étudiants, et éventuellement des enseignants, sont étudiées par des documentalistes gestionnaires de bases de données et des propositions de mots-clés sont faites aux demandeurs pour rechercher les documents (y compris les cartes). Après validation des propositions par les étudiants demandeurs, les recherches sont réalisées et les copies des documents sont envoyées. Les limites de la bande passante d'internet dans certains pays, c'est le cas au Niger et à Madagascar, ajoutées aux limites de la distribution et de la qualité de l'électricité, imposent d'envoyer autant de courriels que de documents en fichiers attachés. En effet, l'envoi de plusieurs documents en pièces jointes à un courriel se traduit en général par le fait que seul le texte du courriel arrive à son destinataire, les fichiers attachés se perdant sur la route d'internet semée de passages dans plusieurs serveurs qui représentent autant de barrières.

Ce sont là des réalités très différentes de celles que nous connaissons en France, et qui imposent donc des méthodes de travail adaptées et des moyens humains conséquents. L'association RAFID souhaite développer des partenariats avec des acteurs institutionnels au nord et au sud pour participer à ce type d'action visant à contribuer à l'accès à l'information et à la transmission des savoirs. En convergence avec les nombreuses actions institutionnelles engagées dans le cadre de conventions et partenariats bilatéraux et multilatéraux, l'action d'associations de type ONG est probablement intéressante, car elle est peu développée dans le domaine de la communication documentaire et de la médiation.

\section{BIBLIOGRAPHIE}

AMELOT Xavier, «L'évaluation par télédétection de la déforestation à Madagascar : comparer ce qui est comparable ", dans XI Journées scientifiques du Réseau télédétection de l'AUF, Télédétection et gestion de l'environnement (Antananarivo, Madagascar, 3-7 novembre 2008), éd. numérique, [s. 1.], [s. n.], 2008. [URL : https://halshs.archives-ouvertes.fr/halshs-00347480]

AMELOT Xavier, MOREAU Sophie, CARRIÈrE Stéphanie M., « Des justiciers de la biodiversité aux injustices spatiales : l'exemple de l'extension du réseau d'aires protégées à Madagascar ", dans Blanchon David, Gardin Jean, Moreau Sophie (dir.), Justices et injustices environnementales, Nanterre, Presses universitaires de Paris-Nanterre, 2012, p. 193-216.

BART François, « Les cahiers d'outre-mer (1948-2015) : bilan, évolutions, perspectives », Les cahiers d'outre mer, $\mathrm{n}^{\circ} 272,2015$, p. 595-601.

HUETZ DE LEMPS Alain, « Les géographes de Bordeaux et l'outre-mer », Les cahiers d'outre-mer, $\mathrm{n}^{\circ} 200$, Bordeaux et l'outre-mer, 1997, p. 541-574. 
QUÉMÉRÉ E., AMELOT Xavier, PIERSON Julie, CROUAU-ROY B., CHIKHI. L., « Genetic data suggest a natural prehuman origin of open habitats in northern Madagascar and question the deforestation narrative in this region ", dans Proceedings of the National Academy of Sciences of the United States of America, éd. numérique, [s. 1.], National Academy of Sciences, 2012. [URL : https://

halshs.archives-ouvertes.fr/halshs-00745221]

VENNETIER Pierre, «Cinquante ans de Cahiers d'outre-mer : essai de bilan scientifique », Les cahiers

d'outre-mer, $\mathrm{n}^{\circ}$ 200, Bordeaux et l'outre-mer, 1997, p. 575-606.

\section{NOTES}

1. Dès 2006, Regards (http://www.regards.cnrs.fr/) a bénéficié de l'appui du très grand équipement (TGE) Adonis du CNRS (devenu TGIR Huma-Num) dans le cadre d'un appel à propositions qui a conduit, avec d'autres partenaires, à la mise en place d'une Archive numérique d'objets et de matériaux iconographiques scientifiques (AOMS) par le Centre national pour la numérisation de sources visuelles (CN2SV). La numérisation et la description des cartes ont été poursuivies avec l'appui de la TGIR Huma-Num dans le cadre du consortium ImaGEO (https://imageo.hypotheses.org, les données techniques sont décrites ci-après). Cet organisme, porté par l'unité mixte de services 3598 associant le CNRS, l'université d'Aix-Marseille et le Campus Condorcet, facilite le tournant numérique de la recherche en sciences humaines et sociales en France. HumaNum met en œuvre un dispositif humain de compétences et une infrastructure technologique (services numériques pérennes) à l'échelle nationale et européenne en s'appuyant sur un important réseau de partenaires et d'opérateurs. Les consortiums thématiques, mis en place par la TGIR, regroupent des acteurs de communautés scientifiques pour produire de manière raisonnée et collective des corpus de sources en facilitant les recommandations scientifiques et les bonnes pratiques technologiques. Pour faciliter la diffusion des données de la recherche produites, Huma-Num a développé un dispositif technologique qui permet le traitement, la conservation, l'accès et l'interopérabilité : il offre ainsi des services numériques dédiés, une plate-forme d'accès unifié (Isidore) et une procédure d'archivage à long terme.

2. MédiHAL est une archive ouverte développée par le CCSD en coopération avec le $\mathrm{CN} 2 \mathrm{SV}$ et en collaboration avec le centre Regards. Cette archive a été depuis intégrée à la plate-forme d'archivage HAL également développée par le CNRS.

3. https://www.socgeobx.fr/

4. F. Bart, « Les cahiers d'outre-mer (1948-2015) : bilan, évolutions, perspectives ».

5. A. Huetz de Lemps, «Les géographes de Bordeaux et l'outre-mer»; P. Vennetier, "Cinquante ans de Cahiers d'outre-mer : essai de bilan scientifique ".

6. https://www.u-bordeaux-montaigne.fr/fr/documentation/vos-bibliotheques/ bibliotheque-de-geographie-cartotheque-centre-de-ressources-de-l-isic.html. Voir aussi la présentation de la cartothèque du département de Géographie de l'université Bordeaux-Montaigne par Philippe Laymond: https://www.youtube.com/watch? $\mathrm{v}=\mathrm{F} 00$ sghDKhoE.

7. http://1886.u-bordeaux-montaigne.fr

8. https://gallica.bnf.fr/edit/und/1886-collections-patrimoniales-numerisees-debordeaux-3-0 
9. X. Amelot, S. Moreau, S. M. Carrière, « Des justiciers de la biodiversité aux injustices spatiales : l'exemple de l'extension du réseau d'aires protégées à Madagascar ».

10. X. Amelot, «L'évaluation par télédétection de la déforestation à Madagascar: comparer ce qui est comparable »; E. Quéméré, X. Amelot, J. Pierson, B. Crouau-Roy, L. Chikhi, "Genetic data suggest a natural prehuman origin of open habitats in northern Madagascar and question the deforestation narrative in this region ».

11. https://medihal.archives-ouvertes.fr/REGARDS-PHOTOS/

12. D'autres fonds documentaires importants existent à Bordeaux, en particulier: le fonds documentaire du Centre d'étude d'Afrique noire (CEAN), devenu en 2011 Les Afriques dans le monde (LAM), lors de la fusion avec le Centre d'études et de recherches sur les pays d'Afrique orientale (CREPAO) de l'université de Pau et des Pays de l'Adour (http://lam.sciencespobordeaux.fr/fr/page/bibliotheque). C'est un fonds important mais il ne contient pas de cartes ni de photographies; il compte actuellement 30000 monographies et 850 titres de revues: il est géré par l'Institut d'études politiques de Bordeaux et diffusé dans le système Babord + et le SUDOC. Il est spécialisé dans les domaines des sciences politiques, administratives et juridiques, de la sociologie, de l'économie, de l'histoire contemporaine et des relations internationales de l'Afrique subsaharienne et particulièrement l'Afrique lusophone et australe. Signalons également: la bibliothèque municipale de Bordeaux qui possède un fonds important sur l'Afrique et l'Amérique latine et a entrepris la numérisation de certains documents; les archives départementales de la Gironde qui conservent aussi des documents en particulier sur l'Afrique dans les domaines administratifs, réglementaires et commerciaux provenant de plusieurs donations; enfin on peut citer d'autres fonds qui n'existent plus, comme celui de l'École internationale de Bordeaux, créée par en 1972 par l'Agence de coopération culturelle et technique, qui comptait une documentation intéressante de photographies concernant l'actualité dans les pays francophones.

13. La description des cartes suit les principes du catalogage et de l'indexation : norme AFNOR «Documents cartographiques", Z 44-067, septembre 1991 (documentation, catalogage des documents cartographiques, rédaction de la notice bibliographique); norme ISO 3166-1 pour le signalement des pays ; norme ISO 639-2b pour le signalement de la langue des unités documentaires décrites; norme ISO 8601 pour le signalement des dates, consignées dans l'instrument de recherche et dans les unités documentaires décrites.

14. Les API (application programming interface) sont des interfaces de programmation permettant de faire un lien entre un logiciel externe et le système cartographique de Google.

15. Dot per inch (point par pouce).

16. L'interopérabilité consiste dans le moissonnage des métadonnées à l'aide du protocole OAI-PMH (open archives initiative protocol for metadata harvesting) selon le vocabulaire Dublin Core simple (DCes).

17. https://www.navigae.fr/

18. http://rafid.hypotheses.org/ 


\section{RÉSUMÉS}

La numérisation de fonds de cartes anciennes par plusieurs centres de recherche, dont le centre d'information scientifique et technique du CNRS Regards (Pessac), et par le consortium « ImaGEO, Images des géographes - Cartes et photographies ", financée par la très grande infrastructure de recherche Huma-Num, permet de sauvegarder ces documents fragiles par la nature de leur support et par leur format, et de faciliter les comparaisons avec des documents plus récents produits par la géomatique et la cartographie 2.0. La description détaillée par des métadonnées facilite aussi la diffusion de plusieurs fonds concernant des pays d'Afrique, d'Amérique et d'Asie pour les chercheurs des pays couverts par ces cartes. D'autres fonds de photographies scientifiques, en particulier de diapositives prises par des chercheurs depuis 1950, sont en cours de numérisation et sont diffusés dans l'archive ouverte MédiHAL : la transmission des savoirs est enrichie par l'indexation détaillée et les annotations par leurs auteurs ou par des documentalistes.

\section{AUTEURS}

\section{DANIEL POUYLLAU}

Ingénieur de recherche au CNRS en retraite

Administrateur de l'association RAFID (Réseau d'acteurs pour la diffusion des fonds

d'information et de documentation pour le développement)

\section{FRANÇOIS BART}

Professeur émérite de l'université Bordeaux-Montaigne

Président d'honneur de la revue Les cahiers d'outre-mer 


\title{
Des catéchismes à Voltaire : le projet MEDIATE et le rôle des livres « moyens » dans la transmission culturelle à l'époque des Lumières
}

\author{
Alicia C. Montoya
}

1 Dans cette contribution, nous présentons le projet MEDIATE (Measuring Enlightenment: disseminating ideas, authors, and texts in Europe), dont le but est d'étudier la circulation transnationale des livres et la transmission des savoirs à l'époque des Lumières (1665-1830). Prenant appui sur un corpus inédit de quelques centaines de catalogues de vente aux enchères de bibliothèques privées, ce projet postule que parmi les livres les plus diffusés au XVIII ${ }^{\mathrm{e}}$ siècle se trouve une catégorie d'ouvrages popularisateurs qui constitue un corpus de textes de culture moyenne. Ce sont ces livres qui, par leur fonction de passerelle intellectuelle, ont permis à un lectorat de sous-élite de faire connaissance avec les nouvelles idées des Lumières, sans pour autant laisser de côté leurs lectures traditionnelles, souvent d'empreinte religieuse. Après avoir décrit les origines du projet, nous nous pencherons sur un certain nombre de traits typiques de ce corpus, et finirons par une présentation de la base de données MEDIATE et de son apport à nos recherches.

\section{Étudier le champ littéraire du XVIII ${ }^{\mathrm{e}}$ siècle}

2 Le projet numérique MEDIATE, financé entre 2016 et 2022 par le Conseil européen de la recherche (ERC) à l'université Radboud (Nimègue, Pays-Bas'1), a pour objectif d'étudier la circulation des livres en Europe au XVIII ${ }^{\mathrm{e}}$ siècle, avec un intérêt particulier pour ceux qui ont été associés au mouvement des Lumières. Àcette fin, nous sommes actuellement en train de développer une base de données qui hébergera un matériau extrait d'un corpus de catalogues de bibliothèques privées vendues aux enchères dans les Provinces-Unies, en France, dans le Royaume-Uni et en Italie entre 1665 et 1830. Notre hypothèse de départ est que, si l'on veut comprendre de façon adéquate la 
diffusion des livres et des idées du mouvement des Lumières - notamment les ouvrages connus des philosophes - ainsi que les processus de mutation intellectuelle et sociale qu'ils auraient inspirés, selon la célèbre thèse de Daniel Mornet ${ }^{2}$, il est essentiel de comprendre le positionnement de ces livres dans un champ culturel plus large. Ces livres et auteurs jouent en effet un rôle non pas de façon isolée, mais sont pleinement intégrés à des réseaux plus vastes les reliant à d'autres livres et auteurs oubliés aujourd'hui, et constituant ensemble un système littéraire. Ce système implique des rapports entre des textes dont le prestige est variable, issus de régions et de contextes linguistiques différents, et plus ou moins éloignés des centres d'autorité culturelle. Comme l'a montré Pascale Casanova dans son étude sur la « république mondiale des lettres ${ }^{3}$ ", ces rapports sont loin d'être toujours équitables, et les textes et auteurs qui accèdent à la reconnaissance officielle le font souvent aux dépens d'autres, moins bien positionnés dans le champ culturel. Dans ce que Franco Moretti a qualifié d'« abattoir de la littérature 4 ", la grande majorité des textes qui ont eu, à un moment donné, une certaine reconnaissance, finissent par être oubliés. Ceci rend exceptionnellement délicate la tâche d'évaluer l'apport - novateur, révolutionnaire, ou au contraire conventionnel - de ceux qui nous sont connus aujourd'hui. Comment comprendre alors le « vaste non-lu » (the great unread), selon la belle expression de Margaret Cohen ${ }^{5}$ ? Une approche quantitative et numérique s'impose vite. Comme le souligne encore Moretti, " un champ si large ne peut pas être compris en cousant ensemble plusieurs fragments d'information sur des cas individuels, car il n'est pas la somme de ces cas individuels : il est un système collectif, qui doit être compris comme tel, comme un ensemble ${ }^{6} »$. Or seule une approche numérique, capable de digérer des données concernant les centaines de milliers de textes publiés au cours d'une époque historique donnée, permet vraiment cette analyse.

\section{À la recherche des auteurs middlebrow}

3 La nécessité d'une approche quantitative pour saisir le champ littéraire du XVIII ${ }^{e}$ siècle est clairement apparue lors d'une étude antérieure que nous avions publiée en 2004 sur la réception des auteurs féminins antérieurs à 1800. Dans le cadre de cette étude, inspirée par la célèbre enquête menée jadis par Daniel Mornet sur un corpus de 500 catalogues de vente de bibliothèques parisiennes ${ }^{7}$, nous avions analysé 254 catalogues de bibliothèques privées vendues aux enchères dans les ProvincesUnies ${ }^{8}$. Nous avions supposé, étant donné le rôle central des Provinces-Unies dans le commerce du livre à cette époque, que ce corpus pourrait aussi fournir des renseignements sur les grandes tendances du marché du livre en Europe. Mais tout comme le sondage de Mornet avait montré que le roman contemporain le plus cité dans la seconde moitié du XVIII ${ }^{\mathrm{e}}$ siècle était les Lettres d'une Péruvienne de $\mathrm{M}^{\mathrm{me}}$ de Graffigny, tandis qu'en revanche Le contrat social n'apparaissait qu'une seule fois dans les catalogues, notre étude néerlandaise a révélé que les ouvrages le plus souvent cités dans nos catalogues de bibliothèques d'élite n'étaient pas ceux des philosophes des Lumières, mais ceux d'un certain nombre d'auteurs pédagogues relativement oubliés aujourd'hui. Le second auteur français du XVIII ${ }^{e}$ siècle le plus cité, après Voltaire, était Marie Leprince de Beaumont, dont les ouvrages étaient recensés dans $50 \%$ de tous les catalogues. Ceux de Rousseau et de Montesquieu, par contraste, figuraient dans $43 \%$ et ceux de Diderot dans $27 \%$. Parmi les auteurs du XviII siècle, la présence la plus élevée 
revenait au couple de journalistes Joseph Addison et Richard Steele, dont l'ouvrage Le spectateur figurait dans $66 \%$ des bibliothèques ${ }^{9}$. À part $\mathrm{M}^{\mathrm{me}}$ Leprince de Beaumont, une deuxième femme de lettres était massivement présente dans les catalogues, le "gouverneur » du duc de Chartres, futur roi Louis-Philippe, Stéphanie-Félicité Du Crest, comtesse de Genlis. Les ouvrages pédagogiques de $\mathrm{M}^{\mathrm{me}}$ de Genlis figuraient dans $32 \%$ des bibliothèques vendues avant 1800 . Mais notre étude n'est pas la seule à pointer l'importance de ces ouvrages. Ces chiffres sont comparables aux résultats d'autres analyses numériques du marché du livre au XviII e siècle plus récentes, notamment la base de données FBTEE (French book trade in Enlightenment Europe), récensant les archives de la Société typographique de Neuchâtel des années 1769-1794. Dans cette base de données, $\mathrm{M}^{\mathrm{me}}$ Leprince de Beaumont figure en sixième place dans la liste des ventes, suivie en huitième place par $\mathrm{M}^{\text {me }}$ de Genlis. Les autres auteurs sont 1 , Voltaire ; 2, Tissot ; 3, Mercier ; 4, Dorat ; 5 , Rousseau ; 7 , Mairobert ${ }^{10}$.

4 En outre, à part les noms de Beaumont et de Genlis, d'autres noms d'auteurs aujourd'hui oubliés figuraient de façon récurrente dans les catalogues de bibliothèque, ce qui paraissait justifier l'hypothèse selon laquelle ces deux femmes de lettres ne seraient que deux représentantes d'un phénomène plus vaste, et que le genre d'ouvrages qu'elles avaient publié aurait bien pu dominer un certain paysage éditorial au XVIII ${ }^{\mathrm{e}}$ siècle. En matière de genre ou de contenu, il était clair que l'apport des livres religieux, aussi bien de gros volumes théologiques que des livres plus modestes de dévotion personnelle, était également massif dans ces catalogues. Mais étant donné les contraintes que constituait notre approche manuelle, qui ne nous permettait de compter que les occurrences d'un nombre restreint de titres, il nous était impossible à cette date de développer des statistiques plus poussées sur ce phénomène. Toutes ces données, prises ensemble, suggéraient toutefois l'existence d'un marché et d'une culture du livre au XVIII ${ }^{e}$ siècle dont nous ne saisissons pas encore toute la complexité, et qu'il faudrait étudier de façon systématique et quantitative, prenant appui sur les possibilités techniques et statistiques que nous offrent les outils informatiques modernes.

\section{Définir la littérature de culture moyenne}

5 L'hypothèse centrale du projet MEDIATE était, d'abord, que ces deux auteurs mis en avant par notre étude de 2004 ne sont pas des cas isolés, mais seraient représentatifs d'un phénomène plus vaste, et que le corpus dont ils font partie regroupe des textes qui pourraient être décrits comme constituant une littérature de culture moyenne dans l'Europe du xvIII siècle. Deuxièmement, le projet postule que ces textes auraient pu jouer un rôle dans la diffusion des idées des Lumières auprès d'un plus grand public, à la fois en transmettant les idées de l'élite à des catégories sociales qui n'en faisaient pas partie, mais aussi en donnant forme à un nouveau lectorat à travers des attitudes culturelles spécifiques.

6 Ces hypothèses sont étayées par le concept d'une culture "moyenne ", tel qu'il a été élaboré en France par Pierre Bourdieu, et dans le monde anglo-saxon par des chercheurs qui se sont penchés sur le phénomène du middlebrow dans la littérature anglophone des $\mathrm{XIX}^{\mathrm{e}}$ et $\mathrm{xx}^{\mathrm{e}}$ siècles ${ }^{11}$. Les ouvrages d'auteurs comme $\mathrm{M}^{\mathrm{me}}$ Leprince de Beaumont et $\mathrm{M}^{\mathrm{me}}$ de Genlis, en effet, ressemblent sur plusieurs points à cette littérature middlebrow, dans sa définition anglo-saxonne ${ }^{12}$. Ils partagent avec elle le succès 
commercial, le discours moralisateur, une vocation popularisatrice et une accessibilité à des publics ne faisant pas partie des élites culturelles, une surreprésentation d'auteurs féminins et un prétendu manque de qualité littéraire, du moins aux yeux des critiques les plus influents. Si le concept du middlebrow, né dans le paysage éditorial du $\mathrm{XIX}^{\mathrm{e}}$ siècle, doit être manié avec précaution en l'appliquant au XVIII ${ }^{\mathrm{e}}$ siècle, il nous aide néanmoins à reconnaître le positionnement particulier de ces textes dans le champ littéraire de leur époque. Car ces textes n'appartiennent ni à la "haute» culture associée aux philosophes et à l'élite intellectuelle, ni à la culture populaire dont font partie des textes comme les catéchismes, les ouvrages de dévotion et la fameuse Bibliothèque bleue. Dans ce "modèle à deux niveaux du marché du livre au XvIII siècle en France » qu'ont évoqué certains chercheurs, « où coexistait un vaste marché du livre traditionnel, particulièrement religieux, à côté d'un marché beaucoup plus limité mais de haute valeur en livres non religieux, dont les ouvrages des Lumières ${ }^{13}$ ", on voit mal où situer ces textes qui ne sont ni l'un ni l'autre. Les ouvrages phares des Lumières, d'un côté, et le livre de colportage et le livre religieux, de l'autre, ont été l'objet d'études ponctuelles ${ }^{14}$. Ce qui manque, c'est une analyse convaincante des rapports entre ces deux types de texte à l'intérieur du système littéraire du xvIII siècle. Comment, en d'autres mots, passait-on des catéchismes populaires à Voltaire? S'agissait-il pour ces deux classes de texte de deux lectorats absolument séparés, ou y avait-il au contraire - comme on l'a montré dans le cas de la Bibliothèque bleue par rapport à la culture des élites ${ }^{15}$ - des passerelles et des points de liaison entre les deux ? Et si certains lecteurs étaient susceptibles de lire les deux types de texte, lecture religieuse et lecture des Lumières, comment se fait-il que l'une des deux soit sortie " gagnante » de la bataille d'idées qui fit rage au XvIII ${ }^{\mathrm{e}}$ siècle?

7 Ce sont autant de questions auxquelles on peut commencer à fournir une réponse si l'on suppose l'existence d'une classe de publications intermédiaires, comprenant des écrits pédagogiques et popularisateurs comme ceux de $\mathrm{M}^{\text {me }}$ Leprince de Beaumont et $\mathrm{M}^{\mathrm{me}}$ de Genlis, ciblant un public provincial de sous-élite, incluant des femmes et des enfants. Ces textes auraient combiné certaines idées développées dans la "haute " littérature des Lumières avec des structures profondes, des stratégies discursives et des traditions textuelles communes avec les textes religieux, d'empreinte plus traditionnelle, voire populaire. Les idées nouvelles des Lumières, selon cette théorie, devaient d'abord être intégrées dans un cadre religieux familier afin de devenir accessibles et acceptables pour un lectorat européen élargi. Le rôle médiateur de ces textes irait donc dans plusieurs sens. Non seulement ils transmettraient les idées de l'avant-garde en direction d'un public plus étendu, mais ils se serviraient aussi de modèles culturels issus de ces mêmes publics dans le cadre d'une réécriture des buts et des aspirations du mouvement des Lumières. Dans cette classe peu étudiée d'auteurs popularisateurs, signalons encore des écrivains comme les abbés Louis-Antoine Caraccioli, Gabriel Gauchat et Noël-Antoine Pluche en France, ou Samuel Formey et le mouvement Philanthropin dans les États allemands. Ils ont tous en commun d'avoir publié des textes à grand succès qui cherchaient à faire passer certaines idées nouvelles des Lumières, souvent exprimées dans un langage vaguement religieux, à un lectorat plus large, éloigné des grands centres d'autorité intellectuelle. Leurs écrits comprennent des succès de librairie comme les Conseils pour former une bibliothèque peu nombreuse mais choisie du pasteur huguenot Samuel Formey (1746), ou le Spectacle de la nature ou Entretiens sur les particularités de l'histoire naturelle de l'abbé Pluche (1732), dans 
lequel un jeune lectorat pouvait faire connaissance avec les acquis les plus récents de l'histoire naturelle, présentés dans un cadre physico-théologique rassurant.

\section{Un phénomène éditorial européen}

8 Le trait le plus facile à mesurer des textes appartenant à cette classe middlebrow est leur succès commercial. La large diffusion de ces écrits montre qu'ils ne devraient pas être étudiés dans un contexte national, mais comme un phénomène éditorial à l'échelle européenne, avec des influences allant du centre parisien aux périphéries régionales et européennes, mais aussi des périphéries au centre. $\mathrm{M}^{\text {me }}$ Leprince de Beaumont, d'origine normande, fait sa carrière en Lorraine et à Londres, où ses ouvrages subissent des influences anglaises, et elle est parrainée par Joseph II d'Autriche et Catherine de Russie. Il est même possible que ces ouvrages aient eu plus de succès en province qu'à Paris, et à l'étranger qu'à l'intérieur du royaume. Ceci complique évidemment nos idées reçues sur l'impact relatif, en dehors de la France, des figures phares qu'étaient les philosophes ${ }^{16}$. Un facteur de ce succès transnational se trouve dans le fait que les ouvrages d'auteurs comme $\mathrm{M}^{\text {me }}$ Beaumont et $\mathrm{M}^{\mathrm{me}}$ de Genlis sont intégrés dans des pratiques pédagogiques à travers l'Europe. De l'Espagne jusqu'en Russie ${ }^{17}$, ces livres fonctionnent comme des outils pour l'apprentissage de la langue française et comme des manuels transmettant les bases de la religion, des sciences, de la géographie et de l'histoire à un nouveau lectorat. Or, depuis l'invention de l'imprimerie, ce sont les textes à usage scolaire qui ont été le moteur de l'édition et qui ont connu les tirages et le nombre de réimpressions les plus élevés.

9 Si l'usage de ces écrits de culture moyenne dans un contexte pédagogique explique leur large diffusion au-delà de la France, leurs auteurs semblent aussi avoir consciemment cherché à cibler un lectorat élargi en mettant tout en œuvre pour les rendre accessibles. Contrairement aux docteurs ou aux savants, $\mathrm{M}^{\mathrm{me}}$ Leprince de Beaumont affirme, non sans fierté :

«Je n'ai nul effort à faire pour me mettre à portée [des lecteurs], c'est mon état naturel; je ne pense rien, je n'écris rien, qu'une personne de bon sens, sans étude, ne puisse écrire ni penser : je ne sais que mon catéchisme, mais je le sais bien ${ }^{18}$.»

Ce manque supposé d'ambition intellectuelle trahit une posture d'auteur qui fait de son statut social une base de contestation intellectuelle. Nous avons étudié ailleurs le rôle qu'a pu jouer la classe sociale des auteurs, voire leur identification aux valeurs d'une certaine bourgeoisie commerçante " moyenne ", dans le processus de dévalorisation ou de valorisation de leur œuvre. Le reproche souvent réitéré selon lequel ces auteurs manqueraient de "style noble" s'explique, selon nous, comme une tentative d'exprimer ce lien troublant entre leurs ouvrages et le positionnement de ces auteurs dans le champ littéraire de l'époque, autant national que transnational, en matière de capital aussi bien social que culturel ${ }^{19}$.

\section{Des livres religieux, des livres conservateurs?}

11 Un deuxième trait qui pourrait expliquer le succès commercial de ces ouvrages de culture moyenne est leur discours moralisateur, d'accent parfois religieux, proche des ouvrages de dévotion populaire qui étaient les livres de chevet de la plupart des lecteurs en dehors des élites culturelles. Les auteurs middlebrow s'inscrivent dans un 
mouvement que les chercheurs américains ont qualifié de religious Enlightenment ou "Lumières religieuses ", correspondant à un courant à l'intérieur du mouvement des Lumières qui, tout en retenant certains éléments des pratiques religieuses traditionnelles, cherche aussi à moderniser l'horizon culturel des croyants ${ }^{20}$. Leurs ouvrages comportent les quatre traits caractéristiques qu'attribue David Sorkin à ces Lumières religieuses: une préoccupation pour la théologie naturelle, une prise de position en faveur de la tolérance religieuse, voire une vision supraconfessionnelle, un grand impact international et un engagement explicite dans la sphère publique, parfois avec le soutien de l'État. Ces textes essaient de la sorte de réconcilier une vision du monde essentiellement religieuse avec les nouvelles idées des philosophes, notamment la valorisation de la raison comme instrument de connaissance privilégié. Les Magasins de $\mathrm{M}^{\text {me }}$ Leprince de Beaumont, collaboratrice pendant sa carrière de certains des scientifiques les plus connus de son époque, fournissent une synthèse des connaissances les plus actuelles, allant des sciences naturelles à la littérature et à la géographie. Bref, ces auteurs prônaient les idéaux des Lumières en matière de raison, de tolérance et d'utilité sociale de la modernité. Mais ils ancraient aussi leur œuvre dans leur foi catholique. Le mouvement des Lumières, dans cette perspective, pouvait aussi laisser une place aux Lumières de la foi ${ }^{21}$.

Dans leur choix d'une perspective religieuse, ces ouvrages de culture moyenne font preuve également de points de vue politiques qu'on pourrait associer aux mouvements qui, au cours du xIX siècle, vont se définir comme conservateurs. Le théoricien anglais du conservatisme, Roger Scruton, a décrit les idées qui définiraient selon lui ces mouvements. Né dans les milieux des antiphilosophes, et témoignant par conséquent d'un esprit anti-Lumières, antirévolutionnaire ou plus globalement antimoderne ${ }^{22}$, le conservatisme politique se distinguerait par ses liens avec la tradition religieuse ; une posture qui valoriserait l'autorité contre la liberté; une conception ordonnée et organique, fondée sur le passé historique, d'une société hiérarchique, un appel au modèle de la famille plutôt qu'au contrat social comme modèle de société; et un déni paradoxal de la politique, qui fait que cette pensée pourtant proprement politique ne se pose pas toujours comme telle ${ }^{23}$. Or ces traits se retrouvent fréquemment dans les textes de certains de ces auteurs que nous avons qualifiés de middlebrow. Nous avons déjà noté l'importance du cadre religieux dans ces écrits. La tradition religieuse soustend à son tour l'idéal ancien d'une société d'ordres, fondée notamment sur la tradition salesienne des états de vie. C'est dans cette optique qu'une Leprince de Beaumont brosse le portrait d'une pieuse servante qui, en se levant, «dira en s'habillant: Mon Dieu, je vous remercie de m'avoir fait servante; j'aime à l'être, parce que c'est votre sainte volonté ; je crois que c'est pour mon bien. En disant ces choses, Marie élève son cœur à Dieu: elle aura accompli le premier commandement, en adorant son Créateur ${ }^{24}$ ». Malgré des prises de position culturellement progressistes, notamment en matière de défense des capacités des femmes, ce texte témoigne aussi résolument en faveur d'un statu quo social et politique lorsqu'il reprend l'identification traditionnelle du pouvoir séculier avec le pouvoir divin :

«Apprenons à nous soumettre sans murmurer, aux lois de notre pays, et croyons qu'elles sont sages. Apprenons à respecter les puissances, parce qu'elles viennent de Dieu. Apprenons que toutes les fois que nous manquons d'obéir aux princes, nous désobéissons à $\operatorname{Dieu}^{25}$. »

13 Le positionnement politiquement conservateur de $\mathrm{M}^{\mathrm{me}} \mathrm{de}$ Genlis, de même, a été souvent remarqué, même si on trouve également chez elle, en même temps, une 
véritable philosophie politique qui nie explicitement tout but politique de sa part. Ainsi, ce conseil à ses lecteurs dans l'épître dédicatoire de son roman Les petits émigrés :

«J'espère, mes enfants, que vous imiterez un jour cette louable modestie, et qu'à 18 ou 20 ans, toujours entièrement dévoués à votre pays et soumis à ses lois, vous

aurez assez d'esprit pour ne pas disserter sur les différentes formes de gouvernement, et pour ne pas vous ériger en législateurs ${ }^{26}$.»

C'est sans doute à la fois à cause de leur couleur politique et de cette "essence inarticulée ${ }^{27}$ » que ces écrits conservateurs n'ont pas encore fait l'objet d'une étude telle qu'ils le mériteraient en tant que textes politiques : il reste évidemment du travail à faire, et nous n'avons pu faire plus ici qu'esquisser quelques pistes à suivre.

\section{La base de données et le projet numérique MEDIATE}

Comment mobiliser cet ensemble d'hypothèses sur la littérature de culture moyenne dans un projet numérique ? La base de données MEDIATE prend comme point de départ un corpus important de catalogues de bibliothèques privées vendues aux enchères tout au long du XVIII ${ }^{e}$ siècle. Le caractère supposément privé de ces collections, en effet, nous permet d'étudier la circulation des idées du point de vue de la réception plutôt que de la production des livres, en nous fournissant des indications sur leurs lecteurs possibles. Tout en restant consciente des dangers d'une approche axée sur la seule possession des livres - un livre en bibliothèque n'équivaut évidemment pas à un livre réellement lu ${ }^{28}$ - nous posons l'hypothèse que la possession d'un livre nous fournit des indications précieuses sur les aspirations intellectuelles du collectionneur, sur l'association de certains groupes sociaux ou professionnels avec certains types de lecture, sur le prestige accordé à certains livres comme forme de capital culturel et sur l'évaluation par les libraires de la valeur monétaire de ces mêmes livres.

Dans une première étape, celle de la collecte des données, le projet utilise des répertoires existants afin de créer un corpus numérique de quelques centaines de catalogues de bibliothèques privées de taille petite ou moyenne et vendues aux enchères entre 1665 et 1830 . Nos recherches couvrent quatre zones géographiques - les Provinces-Unies, le Royaume-Uni, la France et l'Italie - que nous avons choisies en fonction de leur apport au mouvement des Lumières, de la diversité de langues qu'elles recouvrent, et du rôle de ces régions dans les réseaux intellectuels et dans le commerce du livre au XVIII ${ }^{e}$ siècle. Nos sources comprennent notamment les catalogues de vente néerlandais répertoriés et photographiés par Bert Van Selm et ses collaborateurs, actuellement numérisés dans la collection Book sales catalogues online $\left(\mathrm{BSCO}^{29}\right)$, ainsi que les catalogues de bibliothèques privées décrits dans d'autres répertoires tels que ceux de Françoise Bléchet pour la France, et A. N. L. Munby et Lenore Coral pour le Royaume-Uni ${ }^{30}$. Nous axons notre travail de collecte sur les bibliothèques de taille petite ou moyenne, c'est-à-dire celles dont le catalogue cite moins de 1000 lots, car celles-ci devraient nous permettre de cibler les collections de la sous-élite, ou du public de culture moyenne destinataire de la littérature qui nous intéresse ${ }^{31}$. Les transcriptions de ces catalogues et les données que nous en aurons extraites comprenant plus d'un demi-million de notices sur des livres individuels recensés dans les catalogues - seront mises à la disposition des chercheurs dans une base de données en libre accès dès 2022. 
Le schéma conceptuel de notre base de données MEDIATE comprend actuellement huit entités: person (personne), collection, catalogue, heading (catégorie attribuée par le libraire), lot, item (livre ou autre objet à vendre), manifestation (édition du livre) et work (œuvre, pris au sens général ${ }^{32}$ ). Ceci nous permet de mobiliser le concept de littérature middlebrow à plusieurs niveaux, prenant en compte le statut social "moyen " du possesseur (person), le volume de la bibliothèque (collection), ou le caractère middlebrow de l'ouvrage lui-même (work). En outre, en déployant les instruments numériques d'analyse utilisés plus couramment pour décrire les réseaux sociaux, nous pourrons visualiser les liens entre certaines œuvres (work) entre elles, de même que les ouvrages qui se trouvent le plus souvent à proximité les uns des autres et, par là, fournir un modèle idéal des jalons bibliographiques, entre les catéchismes et Voltaire par exemple. La technologie des systèmes d'information géographique (SIG), enfin, nous permettra de visualiser les livres physiques (item) lorsqu'ils parcourent l'Europe, passant de bibliothèque en bibliothèque $\mathrm{e}^{33}$.

Dans une deuxième étape, celle de l'enrichissement des données, nous nous focalisons sur nos objectifs de recherche sur le système littéraire et le lectorat des livres en collectant des données à la fois sur les livres recensés dans les catalogues et sur leurs possesseurs, ceci afin de rendre possible une étude prosopographique de cette population. Notre entité person comprend ainsi plusieurs propriétés, dont la profession et l'appartenance religieuse (person pouvant être non seulement le possesseur de la bibliothèque, mais aussi un auteur, un éditeur, etc.) :

- Nom court

- Rôle*

- Nom de famille

- Prénom

- Date de naissance

- Date de décès

- Sexe*

- Profession*

- Ville de naissance

- Ville de décès

- Ville de résidence

- Enfants

- Époux ou épouse

- En rapport avec (person)

- Affiliation religieuse*

- Auteur de (work)

Chaque propriété marquée par un astérisque correspond à son tour à un thésaurus listant les différents choix. Par exemple, pour définir la propriété profession, le chercheur pourra choisir entre seize possibilités, qui sont susceptibles d'être cumulées. Ces choix reflètent les catégories utilisées dans notre étude antérieure, ainsi que d'autres études bibliométriques comme celle de Michel Marion sur les bibliothèques parisiennes ${ }^{34}$. L'enrichissement des données rendra ainsi possibles des questionnements précis sur les préférences de certaines classes de lecteurs et sur le 
fonctionnement du marché en ce qui concerne la circulation des livres. Le projet alimentera à terme deux bases de données numériques :

- BIBLIO (Bibliography of individually-owned book and library inventories online) comprenant les métadonnées sur tous les catalogues de vente et inventaires de bibliothèques privées britanniques, français, néerlandais et italiens imprimés dans la période 1665-1830 et conservés aujourd'hui. Ces métadonnées concernent essentiellement les entités catalogue et person.

- MEDIATE (Measuring Enlightenment: disseminating ideas, authors, and texts in Europe) comprenant les transcriptions de quelques centaines de catalogues de la période 1665-1830, ainsi que les données extraites de ceux-ci. Ces données concernent essentiellement les entités item (livre physique), person (auteur, possesseur, éditeur, etc.) et work (œuvre).

Nous nous rendons bien compte, enfin, du fait que les catalogues de vente aux enchères ne représentent qu'une seule source - particulièrement riche, certes - concernant le marché du livre au XvIII ${ }^{e}$ siècle, et que les données qu'ils nous livrent comportent des spécificités qui demandent à être maniées avec précaution. Pour mesurer l'impact des ouvrages dans le champ littéraire, il est alors nécessaire de les étudier à travers un ensemble plus vaste de sources, touchant non seulement à la réception mais aussi à la production et à la mise en circulation du livre. C'est pourquoi, dans un troisième et dernier temps, notre projet MEDIATE consolidera les collaborations déjà existantes avec un certain nombre d'autres projets numériques en histoire du livre, sur d'autres types de sources, afin de créer un écosystème de bases de données interopérables. Cette collaboration implique notamment le projet déjà cité de Simon Burrows à l'université Western Sydney (Australie), FBTEE (French book trade in Enlightenment Europe $e^{35}$ ), sur les archives de libraires, et le projet Universal short title catalogue $e^{36}$, à l'université de St Andrews (Écosse), portant sur les catalogues de libraires. L'interopérabilité qui sera établie entre ces différents projets numériques nous permettra de créer un vaste réseau de données portant sur les différents aspects de l'histoire du livre et de fournir ainsi une réponse convaincante à la question de la circulation des livres en Europe, notamment le rôle des livres « moyens » dans la transmission des savoirs à l'époque des Lumières.

\section{BIBLIOGRAPHIE}

ALBERTAN-COPPOLA Sylviane, MCKENNA Antony (dir.), Christianisme et Lumières, Dix-huitième siècle, $\mathrm{n}^{\circ} 34,2002$.

BLÉCHET Françoise, Les ventes publiques de livres en France (1630-1750) : répertoire des catalogues conservés à la Bibliothèque nationale, Oxford, Voltaire Foundation, 1991.

BLOM Helwi, JAGERSMA Rindert, REBOUL Juliette, « Printed private library catalogues as a source for the history of reading in seventeenth and eighteenth-century Europe ", dans Hammond Mary (dir.), The Edinburgh history of reading : early readers, Édimbourg, Edinburgh University Press, 2020, p. 249-269. 
BOLUFER Mónica, « Conversations from a distance : Spanish and French eighteenth-century women writers », dans Ros Xon de, Hazbun Geraldine (dir.), A companion to Spanish women's studies, Woodbridge, Tamesis, 2011, p. 175-188.

BOURDIEU Pierre, Les règles de l'art : genèse et structure du champ littéraire, Paris, Seuil, 1992.

BROWN Erica, GROVER Mary (dir.), Middlebrow literary cultures : the battle of the brows (1920-1960), New York, Palgrave Macmillan, 2012.

BURROWS Simon, The French book trade in Enlightenment Europe, II, Enlightenment bestsellers, Londres, Bloomsbury, 2018.

CASANOVA Pascale, La république mondiale des lettres, Paris, Seuil, 1999.

CHARON Annie, PARINET Élisabeth (dir.), Les ventes de livres et leurs catalogues (XVII ${ }^{e}-\mathrm{XX}^{e}$ siècle), Paris, École des chartes, 2000.

CHARTIER Roger, « Lectures et lecteurs "populaires" de la Renaissance à l'âge classique ", dans Chartier Roger, Cavallo Guglielmo (dir.), Histoire de la lecture dans le monde occidental, Paris, Seuil, 1997, p. 337-354.

COHEN Margaret, The sentimental education of the novel, Princeton, Princeton University Press, 1999. COMPAGNON Antoine, Les antimodernes : de Joseph de Maistre à Roland Barthes, Paris, Gallimard, 2005. DARNTON Robert, The forbidden best-sellers of pre-revolutionary France, New York, Norton, 1996. GENLIS Stéphanie-Félicité de, Les petits émigrés ou correspondance de quelques enfans, Hambourg, [s. n.], 1798, 3 vol.

JAGERSMA Rindert, « Dutch printed private library sales catalogues (1599-1800) : a bibliometric overview », dans Der Weduwen Arthur, Kemp Graeme, Pettegree Andrew (dir.), Book trade catalogues in early modern Europe, Leiden, Brill, p. 87-117.

LEHNER Ulrich L., The Catholic Enlightenment : the forgotten history of a global movement, Oxford, Oxford University Press, 2016.

LEPRINCE DE BEAUMONT Marie, Le magasin des pauvres, artisans, domestiques et gens de la campagne, Lyon, Pierre Bruyset, 1775, 2 vol.

LEPRINCE DE BEAUMONT Marie, Les Américaines ou la preuve de la religion chrétienne par les lumières naturelles, Annecy, C. M. Durand, 1769, 6 vol.

LOH Gerhard, Die Europäischen Privatbibliotheken und Buchauktionen, Leipzig, Loh, 1997-2005, 7 vol. MARION Michel, Collections et collectionneurs de livres au XVIII siècle, Paris, Champion, 1999.

MARTIN Philippe, Une religion des livres (1640-1850), Paris, Cerf, 2003.

MASSEAU Didier, Les ennemis des philosophes : l'antiphilosophie au temps des Lumières, Paris, Albin Michel, 2000.

MCMAHON Darrin, Enemies of the Enlightenment : the French Counter-Enlightenment and the making of modernity, Oxford, Oxford University Press, 2001.

MONTOYA Alicia C., « French and English women writers in Dutch library auction catalogues (1700-1800) : some methodological considerations and preliminary results », dans Van Dijk Suzan, Broomans Petra, Van der Meulen Janet, Van Oostrum Pim (dir.), «I have heard about you » : foreign women's writing crossing the Dutch border, Hilversum, Verloren, 2004, p. 182-216. 
MONTOYA Alicia C., «Livre de piété ou roman? Sur quelques ouvrages pédagogiques de $\mathrm{M}^{\text {mes }}$ Leprince de Beaumont et Genlis », dans Tremblay Isabelle (dir.), Les Lumières catholiques et le roman français, Oxford, Voltaire Foundation, 2019, p. 123-152.

MonToy Alicia C., « Madame Leprince de Beaumont et la littérature "médiocre" (middlebrow)», dans Seth Catriona, Von Kulessa Rotraud (dir.), Une éducatrice des Lumières : Marie Leprince de Beaumont, Paris, Classiques Garnier, 2018, p. 205-226.

MONTOYA Alicia C., « Marie Leprince de Beaumont (1711-1780) : a popular religious pedagogue », dans Lehner Ulrich L. (dir.), Women, Enlightenment and Catholicism : a transnational biographical history, Londres, Routledge, 2018, p. 22-34.

MONTOYA Alicia C., GELDER Wyneke de, « The view from the periphery : French pedagogy and Enlightenment in Russia (Leprince de Beaumont's Magasin des enfants) », De achttiende eeuw, vol. XLV, n 2, 2012, p. 227-253.

MORETTI Franco, Graphs, maps, trees : abstract models for literary history, Londres, Verso, 2005. MORETTI Franco, «The slaughterhouse of literature », MLQ : Modern language quarterly, vol. LXI, $\mathrm{n}^{\circ} 1,2000$, p. 207-227.

MORNET Daniel, « Les enseignements des bibliothèques privées (1750-1780) », Revue d'histoire littéraire de la France, $17^{\mathrm{e}}$ année, ${ }^{\circ}$ 3, 1910, p. 449-496.

MORNET Daniel, Les origines intellectuelles de la Révolution française (1715-1787), Paris, Armand Colin, 1933.

MUNBY A. N. L., CORAL Lenore, British book sale catalogues (1678-1800) : a union list, Londres, Mansell, 1977.

POLLENTIER Caroline, « Configuring middleness : Bourdieu, l'art moyen and the broadbrow », dans Brown Erica, Grover Mary (dir.), Middlebrow literary cultures : the battle of the brows (1920-1960), New York, Palgrave MacMillan, 2011, p. 37-51.

RUBIN Joan Shelley, The making of middlebrow culture, Chapel Hill, University of North Carolina Press, 1992.

SCRUTON Roger, The meaning of conservatism, $3^{e}$ éd., South Bend, St. Augustine's Press, 2002.

SORKIN David, The religious Enlightenment : Protestants, Jews, and Catholics from London to Vienna, Princeton, Princeton University Press, 2008.

VAN DIJK Suzan, MONTOYA Alicia C., « Madame Leprince de Beaumont, Mademoiselle Bonne en hun Nederlandse lezers ", De achttiende eeuw, vol. XXXIV, $n^{\circ}$ 1, 2002, p. 5-32.

VAN SELM Bert, GRUYS J. A., KOOKER H. W. de, continué par BOSTOEN Karel, LANKHORST Otto, MONTOYA Alicia C., VAN DELFT Marieke (dir.), Book sales catalogues online : book auctioning in the Dutch Republic (ca. 1500-ca. 1800), éd. numérique, Leyde, Brill, 2005. [URL : https:// primarysources.brillonline.com/browse/book-sales-catalogues-online]

\section{NOTES}

1. Ce projet a bénéficié d'une subvention du Conseil européen de la recherche (ERC) dans le cadre du programme de recherche et innovation de l'Union européenne 
Horizon 2020 sous la convention $\mathrm{n}^{\circ}$ 682022. Voir aussi le site du projet: www.mediate18.nl.

2. D. Mornet, Les origines intellectuelles de la Révolution française (1715-1787) ; R. Darnton, The forbidden best-sellers of pre-revolutionary France.

3. P. Casanova, La république mondiale des lettres.

4. F. Moretti, « The slaughterhouse of literature ».

5. M. Cohen, The sentimental education of the novel, p. 3.

6. F. Moretti, Graphs, maps, trees: abstract models for literary history, p. 4 (trad. de l'auteur).

7. D. Mornet, « Les enseignements des bibliothèques privées (1750-1780) ».

8. A.C. Montoya, «French and English women writers in Dutch library auction catalogues (1700-1800) : some methodological considerations and preliminary results ».

9. S. Van Dijk, A. C. Montoya, « Madame Leprince de Beaumont, Mademoiselle Bonne en hun Nederlandse lezers ».

10. S. Burrows, The French book trade in Enlightenment Europe, II, Enlightenment bestsellers, p. 68.

11. P. Bourdieu, Les règles de l'art: genèse et structure du champ littéraire; C. Pollentier, "Configuring middleness : Bourdieu, l'art moyen and the broadbrow ».

12. E. Brown, M. Grover (dir.), Middlebrow literary cultures: the battle of the brows (1920-1960) ; J. S. Rubin, The making of middlebrow culture. Pour une application de ce concept au XVIII ${ }^{\mathrm{e}}$ siècle, voir A.C. Montoya, "Madame Leprince de Beaumont et la littérature "médiocre" (middlebrow) ».

13. S. Burrows, The French book trade in Enlightenment Europe, II, Enlightenment bestsellers, p. 154 (trad. de l'auteur).

14. La bibliographie sur les ouvrages des Lumières est vaste et bien connue. Sur le livre religieux, voir P. Martin, Une religion des livres (1640-1850).

15. R. Chartier, « Lectures et lecteurs “populaires” de la Renaissance à l'âge classique ».

16. C'est ce que suggèrent nos données néerlandaises et les données suisses que rapporte S. Burrows dans The French book trade in Enlightenment Europe, II, Enlightenment bestsellers.

17. M. Bolufer, «Conversations from a distance: Spanish and French eighteenthcentury women writers »; A. C. Montoya, W. de Gelder, « The view from the periphery : French pedagogy and Enlightenment in Russia (Leprince de Beaumont's Magasin des enfants) ».

18. M. Leprince de Beaumont, Les Américaines ou la preuve de la religion chrétienne par les lumières naturelles, t. VI, p. 284.

19. A. C. Montoya, "Madame Leprince de Beaumont et la littérature "médiocre" (middlebrow)».

20. S. Albertan-Coppola, A. McKenna (dir.), Christianisme et Lumières ; U. L. Lehner, The Catholic Enlightenment : the forgotten history of a global movement ; D. Sorkin, The religious Enlightenment : Protestants, Jews, and Catholics from London to Vienna.

21. Pour une analyse de l'élément religieux chez $M^{\text {me }}$ Leprince de Beaumont et $M^{\text {me }}$ de Genlis, voir A.C.Montoya, «Livre de piété ou roman? Sur quelques ouvrages 
pédagogiques de $\mathrm{M}^{\text {mes }}$ Leprince de Beaumont et Genlis »; id., "Marie Leprince de Beaumont (1711-1780) : a popular religious pedagogue ».

22. D. Masseau, Les ennemis des philosophes: l'antiphilosophie au temps des Lumières; D. McMahon, Enemies of the Enlightenment: the French Counter-Enlightenment and the making of modernity; A. Compagnon, Les antimodernes: de Joseph de Maistre à Roland Barthes.

23. R. Scruton, The meaning of conservatism.

24. M. Leprince de Beaumont, Le magasin des pauvres, artisans, domestiques et gens de la campagne, t. I, p. 140-141.

25. Ibid., p. 301.

26. S.-F. de Genlis, Les petits émigrés ou correspondance de quelques enfans, t. I, p. XII.

27. Selon l'expression de R. Scruton, The meaning of conservatism, p. 1.

28. Sur les précautions à prendre en utilisant comme source les catalogues de vente des bibliothèques privées, voir H. Blom, R. Jagersma, J. Reboul, "Printed private library catalogues as a source for the history of reading in seventeenth and eighteenth-century Europe ».

29. Dont des catalogues conservés aux Pays-Bas, en Allemagne, au Royaume-Uni, en France et en Russie. Pour la liste complète, voir B. Van Selm et al. (dir.), Book sales catalogues online : book auctioning in the Dutch Republic (ca. 1500-ca. 1800).

30. F. Bléchet, Les ventes publiques de livres en France (1630-1750) : répertoire des catalogues conservés à la Bibliothèque nationale; A. N. L. Munby, L. Coral, British book sale catalogues (1678-1800): a union list; G. Loh, Die Europäischen Privatbibliotheken und Buchauktionen. Voir aussi A. Charon, É. Parinet, Les ventes de livres et leurs catalogues (XVII ${ }^{e}-\mathrm{XX}{ }^{e}$ siècle).

31. Étant donné notre estimation selon laquelle $50 \%$ des catalogues de bibliothèques recensent 1000 lots ou moins. Voir R. Jagersma, «Dutch printed private library sales catalogues (1599-1800) : a bibliometric overview ».

32. En distinguant trois niveaux pour identifier nos livres (work, manifestation, item), nous reprenons le modèle FRBR (functional requirements for bibliographical records). Nous laissons de côté, pour des raisons pratiques, le niveau expression.

33. Pour l'instant, il s'agit de simples cartes permettant de visualiser la distribution géographique d'un titre, des ouvrages d'un auteur ou des lieux de publication des ouvrages faisant partie d'une bibliothèque.

34. M. Marion, Collections et collectionneurs de livres au XVIII e siècle.

35. S. Burrows (dir.), French book trade in Enlightenment Europe ( http:// fbtee.uws.edu.au/).

36. A. Pettegree (dir.), Universal short title catalogue (http://www.ustc.ac.uk/). 


\section{RÉSUMÉS}

Cet article présente un projet numérique financé par le Conseil européen de la recherche, MEDIATE (Measuring Enlightenment : disseminating ideas, authors, and texts in Europe), dont le but est d'étudier la circulation transnationale des livres à l'époque des Lumières (1665-1830). Prenant appui sur un corpus inédit de quelques centaines de catalogues de vente aux enchères de bibliothèques privées, il suppose que parmi les livres les plus diffusés se trouve une catégorie d'ouvrages popularisateurs constituant un corpus de textes de culture moyenne (middlebrow). En tant que passerelle intellectuelle, ces livres ont permis à un lectorat de sous-élite de faire connaissance avec les nouvelles idées des Lumières, sans pour autant abandonner ses lectures traditionnelles, d'empreinte religieuse. Après avoir décrit les origines du projet, nous examinons les traits typiques de ce corpus pour aboutir à une présentation de la base de données MEDIATE et de son apport à nos recherches.

\section{AUTEUR}

\section{ALICIA C. MONTOYA}

Professeur de littérature française, université Radboud (Nimègue, Pays-Bas) 


\title{
Le monument pour transmettre, la carte postale pour diffuser : de la collection à la base de données
}

\author{
France Debuisson-Lagneau
}

1 Collectionner les cartes postales peut sembler de peu de rapport avec la transmission d'un savoir. Sauf à rencontrer les bonnes personnes au bon moment et faire d'une passion personnelle une œuvre collective à large diffusion.

2 Cette histoire a commencé en 1968 lorsque ma mère Roxane Debuisson, grande collectionneuse sur Paris et cartophile elle-même, a donné à l'adolescente que j'étais alors une boitte contenant des cartes postales anciennes de statues de province achetées au cours de ses recherches sur Paris et n'entrant pas dans sa collection. Ce premier noyau, je l'ai organisé, agrandi, enrichi. Bien sûr, j'ai acheté de nouvelles cartes, trouvé de nouveaux monuments et j'ai systématiquement dépouillé les Guides bleus Hachette pour des informations complémentaires comme les emplacements précis des monuments dans la ville, les personnages représentés ou même les noms des sculpteurs souvent précisés dans ces pages. Mon grand plaisir était de trouver des cartes du même monument sous des angles différents et de pouvoir tourner autour, comme si j'étais projetée dans l'espace et dans le temps.

Quelques années et quelques centaines de cartes plus tard, en 1978, j'ai eu l'occasion de me rendre à La Rochelle où se trouve le monument à Eugène Fromentin par Ernest Dubois (1905), que je connaissais par la carte postale et dont j'affectionnais particulièrement la fougue (fig. 1). J'ai pris alors des photos de la statue (et de toutes celles de La Rochelle, bien sûr) et les ai intégrées à ma collection. J'ai ainsi commencé à doubler ma collection d'images anciennes d'images récentes et je ne partais plus en province sans mon appareil photo. 
Fig. 1. - Eugène Fromentin par Ernest Dubois (La Rochelle).

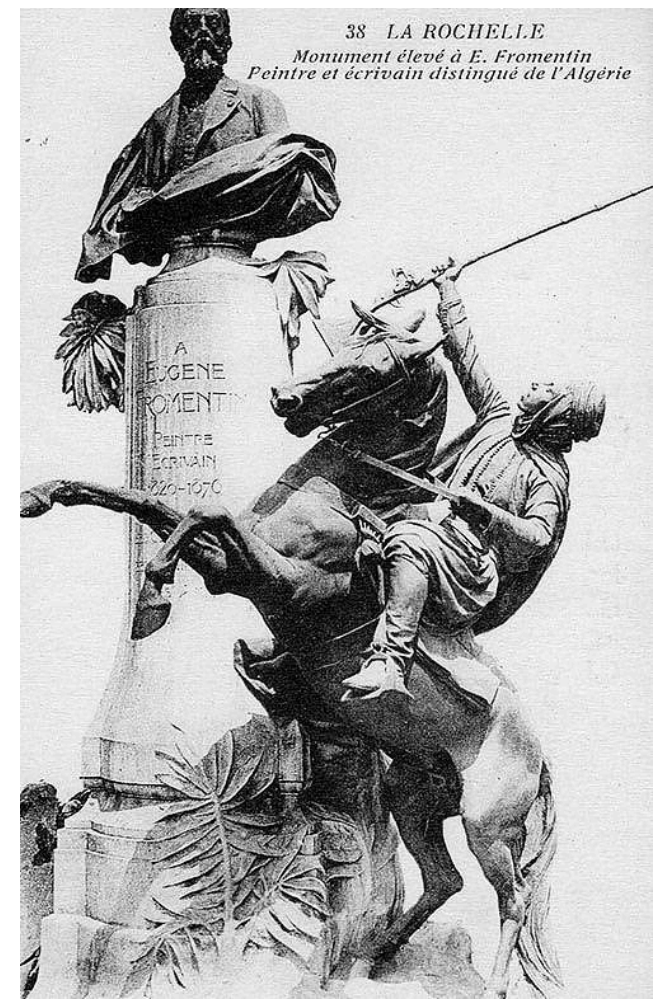

Carte postale.

Collection France Debuisson-Lagneau.

4 Puis, en 1984, j'ai rencontré Anne Pingeot venue faire une recherche sur les monuments parisiens dans la collection de ma mère. Elle a immédiatement vu dans mes cartes classées par sujet le complément du fichier classé par sculpteurs qu'elle préparait pour la documentation du musée d'Orsay. L'association entre un établissement public et un fonds privé a alors commencé au service de la sculpture du XIX ${ }^{\mathrm{e}}$ siècle.

\section{Cartes postales et illustres}

5 La carte postale n'est qu'un petit carton rigide de 9 centimètres sur 14 centimètres et d'environ 4 grammes. C'est dire combien elle est fragile et éphémère. Et pourtant, des millions d'entre elles ont traversé le temps jusqu'à nous.

6 La première carte postale illustrée date de l'Exposition universelle de 1889 et commémore l'érection de la tour Eiffel. L'invention de la phototypie en 1894 a fait passer la production de 52 millions de cartes en 1900 à 123 millions en 1910. C'est ce qu'on a appelé l'âge d'or de la carte postale. À la fois transmission d'images et moyen de communication très bon marché (l'envoi d'une carte coûtait 10 centimes, 5 centimes si vous n'inscriviez que cinq mots coté correspondance), elle reflète tous les aspects de la vie quotidienne des Français du début du siècle dernier. Tout est photographié, les lieux, les fêtes, les marchés... et les monuments.

7 S'il y a autant de cartes postales de monuments, c'est parce que l'âge d'or de la carte postale suit de près l'âge d'or du monument public à la fin du XIX siècle, mouvement 
que Maurice Agulhon a qualifié de statuomanie ${ }^{1}$. Pour la Troisième République, le monument permet à la fois de rendre hommage à ses grands hommes et de transmettre des informations et des messages sur ceux-ci.

8 J'ai défini et organisé ma collection en 3 grandes catégories :

- les grands hommes d'abord (autour de 3500 monuments dans ma base). Cela va de Napoléon à Pierrounet, le rebouteux immortalisé à Nasbinals en 1909. Toutes les communes veulent élever un monument à leur grand homme, célèbre ou local. Ce classement permet de voir d'un seul coup d'œil les 17 monuments à Pasteur. Pour l'anecdote, le grand homme le plus statufié en France est... une femme. J'ai recensé plus de 161 représentations de Jeanne d'Arc. Parfois même elle surplombe le monument aux morts comme à Benestroff ;

- les monuments aux morts des guerres de 1870 et 1914-1918 (2 300 monuments dont près de 300 pour la guerre de 1870). Garder le souvenir des héros, inscrire leur nom dans la pierre est une nécessité nationale et donne lieu après 1870 à des œuvres impressionnantes comme à Limoges (œuvre d'Adolphe Thabard) ou des œuvres majeures comme le Gloria Victis d'Antonin Merciéz. Pour la commémoration de la Grande Guerre et ses millions de morts, les monuments sculptés se font encore plus nombreux. Le poilu est largement glorifié. Il existe même des catalogues pour que chaque commune puisse acheter son monument. Les modèles existants sont signés Étienne Camus, Charles-Henri Pourquet ou Eugène Bénet et son poilu aux lauriers dont j'ai trouvé plus de 50 exemplaires dans toute la France (fig. 2) ${ }^{3}$. On peut également consulter, pour les monuments aux morts de 14-18, la passionnante base de l'université de Lille qui en a fait un inventaire pour la région Hauts-de-France et l'étend aujourd'hui à toute la France ${ }^{4}$; 
Fig. 2. - Monument aux morts de 14-18 par Eugène Bénet (Domfront).

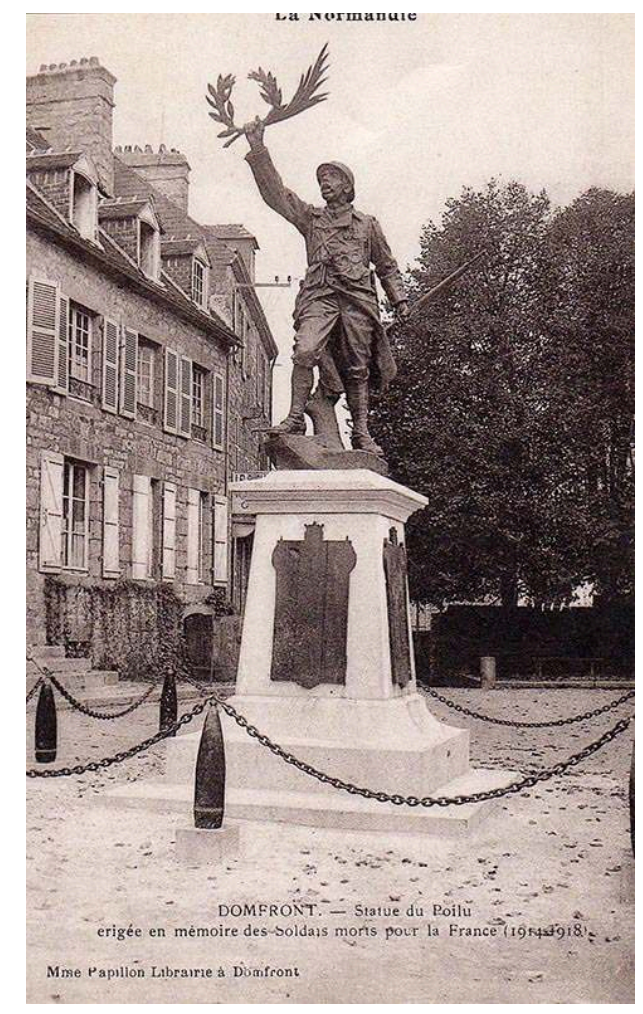

Carte postale.

Collection France Debuisson-Lagneau.

- enfin l'hommage aux idées matérialisées dans la pierre ou le bronze : la statuaire décorative (Le Chêne et le Roseau par Henri Coutheillas à Limoges, 1909), la statuaire allégorique (L'Electricité par André Laoust à Douai, 1904), la statuaire commémorative (La Défense de SaintQuentin par Corneille Theunissen, 1897), la statuaire utilitaire comme sur les fontaines qui permettait de représenter le nu féminin, fort apprécié à l'époque, jusque sur la place publique (la Fontaine des Danaïdes à Marseille par Jean-Baptiste Hugues, 1907).

\section{La carte postale comme recours aux monuments défaillants}

9 L'intérêt de cette base pour les chercheurs tient également à un événement majeur de l'histoire des monuments publics. Je veux parler de la mobilisation des statues qui a fait disparaître près de 1000 monuments en bronze entre 1941 et 1944 sous le couvert d'une récupération des métaux non ferreux.

Par la loi du 11 octobre 1941, le gouvernement de Vichy a réduit les grands hommes de la république à leur poids en bronze, joli règlement de comptes idéologique qui faisait place nette aussi bien sur les places publiques que dans les mémoires collectives.

11 Ainsi ont disparu: Jules Grévy à Dole par Alexandre Falguière (1893), Barbès à Carcassonne par le même (1886), Thiers par Antonin Mercié à Saint-Germain-en-Laye (1880), Fallières par Daniel Bacqué qui ne sera admiré que quatre ans par les habitants de Mézin car érigé en 1938, ou encore Maurice Berteaux par Charles Maillard à Chatou (1922). Le Victor Hugo par Louis-Ernest Barrias et André-Joseph Allar, qui ornait la 
place Victor-Hugo à Paris depuis 1902, disparaîtra à jamais en 1941 : grand homme, allégories et bas-reliefs. Quant à Chappe et son télégraphe érigé au coin de la rue du Bac et du boulevard Saint-Germain, œuvre d'Ernest Damé de 1893, il me manque toujours (fig. 3)!

Fig. 3. - Claude Chappe par Ernest Damé (Paris).

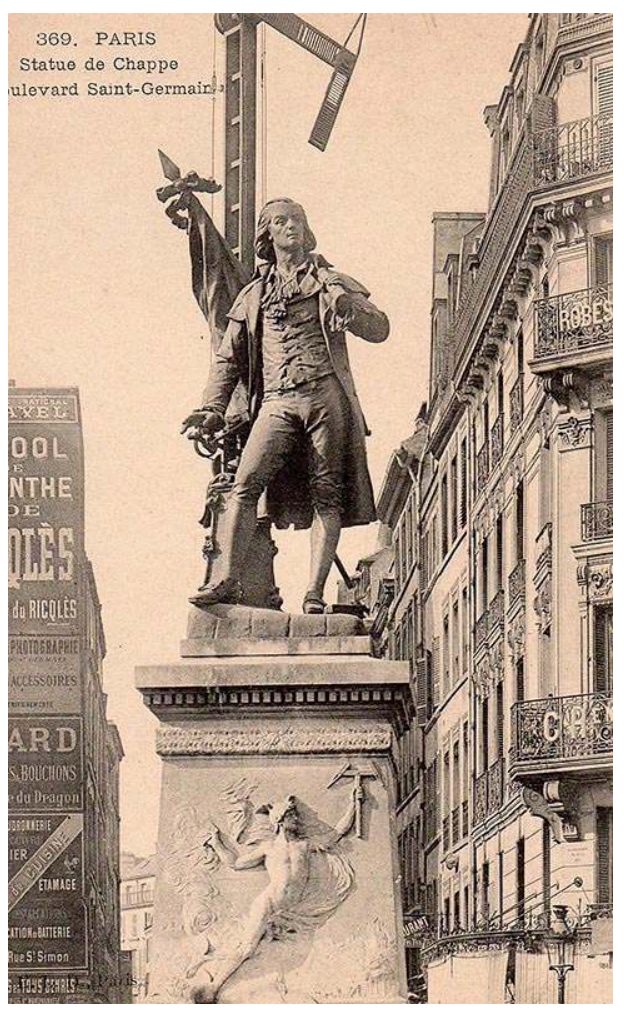

Carte postale.

Collection France Debuisson-Lagneau.

Après la guerre, certaines communes décident de réparer l'outrage fait à leur grand homme et de remplacer le monument. Certains à l'identique, comme Rotrou à Dreux par Jean-Jules Allasseur en 1867. Robert Delandre en fait un moulage en 1942, afin de le remplacer, dès 1943, par une copie à l'identique en pierre. D'autres en faisant appel à un nouveau sculpteur, comme pour l'amiral Tourville à Coutances, œuvre d'Ernest Hulin inaugurée en 1907 et fondue en 1942. Il est remplacé en pierre par l'œuvre du sculpteur Hubert Yencesse et installé à quelques mètres de son piédestal sculpté d'origine, remonté contre un mur. À Sées, de la statue en pied de Conté, chimiste et inventeur du crayon, œuvre de Jules Droz érigée en 1852, il ne reste que la tête qui fut découpée et mise à l'abri en 1941 lorsque le gouvernement de Vichy réclamait du bronze à toutes les communes de France.

\section{Vers la base de données}

En décembre 1986 lorsque ouvre le musée d'Orsay, la collaboration avec l'équipe sculpture est renforcée avec l'arrivée d'Antoinette Le Normand-Romain, Laure de Margerie et Catherine Chevillot et la mise sur informatique s'intensifie de 1987 à 1996. 
En 2002, un contrat de recherche est signé entre l'Institut national d'histoire de l'art, le musée d'Orsay et moi-même pour la production d'un double CD-ROM intitulé À nos grands hommes ${ }^{5}$. Coordonné par Catherine Chevillot et Chantal Georgel et réalisé par Laurent Chastel, il voit le jour en 2004. Le premier CD-ROM, intitulé Chercher, permet de consulter le corpus des 5500 monuments; le second, Comprendre, à visée plus didactique, éclaire différents thèmes à travers une trentaine de vidéos de quelques minutes conçues à partir des cartes postales.

En 2013, un projet de mise en ligne prend forme avec l'équipe de recherche de l'École du Louvre et le LABEX « Les passés dans le présent : histoire, patrimoine, mémoire » de l'université Paris-Nanterre en collaboration avec Claire Barbillon, alors maître de conférences HDR, et aujourd'hui directrice de l'École du Louvre. La base contient à ce jour plus de 7000 monuments et elle est en constante évolution. Bien sûr, je continue à acheter des cartes postales, à parcourir la France. L'enrichissement de la base par des compléments d'information sur les fiches existantes a permis d'effectuer en 2018 une mise en ligne à la disposition de tous, chercheurs comme simples curieux ${ }^{6}$.

Réunir pour transmettre, tel a toujours été le but de cette collection qui a permis de fédérer des énergies et des compétences, de partager des savoirs, des images... et beaucoup de bons moments!

L'érection d'un monument public à un grand homme a été, au XIX et au Xx $x^{e}$ siècle, une forme de transmission de mémoire et de savoirs à l'intention du promeneur, de tous les citoyens, de tout un chacun. Aujourd'hui, l'espace public et la transmission des informations subissent une dilatation extraordinaire grâce à internet. Je souhaite que cette base de données puisse raconter toutes les péripéties et les histoires des monuments publics et apporter autant de plaisirs et de connaissances à ses utilisateurs qu'à sa fondatrice... J'espère également que cette contribution vous incitera vivement à aller voir dans vos caves s'il n'y a pas quelques cartes postales, et à regarder d'un autre œil les statues dans la rue !

\section{BIBLIOGRAPHIE}

AGULHON Maurice, «La statue de grand homme : critique politique et critique esthétique », Mil neuf cent : revue d'histoire intellectuelle, vol. XXI, $\mathrm{n}^{\circ} 1,2003$, p. 9-19. DEBUISSON France, CHEVILLOT Catherine, GEORGEL Chantal et al., À nos grands hommes : la sculpture publique française jusqu'à la Seconde Guerre mondiale, éd. numérique (CD-ROM), Paris, Musée d'Orsay / Institut national d'histoire de l'art, 2004. 


\section{NOTES}

1. M. Agulhon, « La statue de grand homme : critique politique et critique esthétique ».

2. Cette œuvre créée en 1872 au lendemain de la défaite servira de monument aux morts dans six villes de France, érigés à des dates bien plus tardives (Paris, 1879 ; Agen, 1885 ; Bordeaux, 1886 ; Niort, 1881 ; Châlons-sur-Marne, 1892 ; Cholet, 1902).

3. Je renvoie à la très belle arnaque aux monuments aux morts racontée dans Au revoir là-haut, roman de Pierre Lemaitre paru en 2013 et adapté au cinéma par Albert Dupontel.

4. https://monumentsmorts.univ-lille.fr/

5. F. Debuisson, C. Chevillot, C. Georgel et al., À nos grands hommes : la sculpture publique française jusqu'à la Seconde Guerre mondiale.

6. https://anosgrandshommes.musee-orsay.fr/

\section{RÉSUMÉS}

En 1969, j'ai commencé une collection de cartes sur le thème des monuments publics sculptés en France. En complément, j'ai photographié sur le terrain la présence, l'absence et l'état des monuments. Anne Pingeot, qui préparait l'ouverture du département des sculptures au musée d'Orsay, s'est émerveillée de découvrir ma collection qui complétait ses recherches, le musée s'attachant aux artistes et moi aux sujets. D'autres chercheurs, en particulier américains, ont utilisé mon fonds. La collaboration avec l'équipe du musée ouvert en 1986 a débouché sur la signature d'un contrat de recherche en 2002, la parution d'un double CD-ROM en 2004 et un projet de mise en ligne en 2013. C'est cette aventure que je vous propose de présenter car elle intéresse la transmission de l'histoire, de la géographie, de l'urbanisme et de l'art, par une collaboration étroite entre des spécialistes de l'histoire de l'art et une collectionneuse d'images.

\section{AUTEUR}

\section{FRANCE DEBUISSON-LAGNEAU}

Collectionneuse

Conseillère en développement personnel 


\title{
Un plaidoyer pour la transmission des bibliothèques privées des intellectuels et enseignants : la bonne fortune du numérique
}

\author{
Odile Jacquemin
}

1 La bibliothèque privée d'intellectuel a un double statut de patrimoine matériel et immatériel, elle est la trace du cheminement de sa pensée, du contexte et du cadre culturel de l'élaboration du savoir en jeu. L'ère du numérique et des bibliothèques virtuelles permet aujourd'hui de valoriser la valeur d'ensemble d'une bibliothèque, indépendamment de la valeur vénale de chaque ouvrage. Cette contribution propose un plaidoyer et un apport méthodologique à partir d'un témoignage personnel relatif à une douzaine de bibliothèques à transmettre.

\section{L'origine du propos}

2 J'ai été personnellement concernée dans le devenir de la bibliothèque de travail d'un de mes enseignants de l'École nationale supérieure d'architecture de Paris-La Villette, alors unité pédagogique $\mathrm{n}^{\circ} 6$ (UP6), Robert Joly, disparu en 2012, et dont l'association MALTAE (Mémoire à lire, territoire à l'écoute ${ }^{1}$ ) a partagé avec le Centre d'archives d'architecture $\mathrm{du} \mathrm{xx}^{\mathrm{e}}$ siècle ${ }^{2}$ la conservation des quelques centaines d'ouvrages. J'étais aussi présente, avec David Peyceré, le responsable du Centre d'archives d'architecture du $\mathrm{xx}^{\mathrm{e}}$ siècle à la Cité de l'architecture, en 2013 lors de l'hommage rendu à un autre enseignant de cette institution, Michel Vernes, qui comptait parmi ses créateurs. Tout au long de la journée, sa bibliothèque fut au centre des prises de parole et des témoignages : Michel Vernes était bien connu pour l'importance qu'il accordait aux livres et aux lectures dans son enseignement. Je posai alors, par curiosité, la question $\mathrm{du}$ devenir de cette fameuse bibliothèque, question qui resta sans réponse. Il me semblait, ce jour-là, un peu naïvement que cela ne devait pas être si sorcier d'offrir un stage et un bon scanner à un étudiant de l'école pour relever cette bibliothèque, 
photographies à l'appui, avant qu'elle ne soit dispersée, si tel devait être son sort. La bonne fortune du numérique était précisément de le permettre techniquement. Depuis cinq ans, j'avais à entendre parler de nombreuses fois de bibliothèques en mal de devenir, peut-être parce que mon esprit était en alerte sur le sujet, mais plus certainement pour son actualité.

3 La justification de cette communication était donc trouvée : quel meilleur lieu qu'un congrès planchant sur la transmission des savoirs pour évoquer ce vrai sujet et en faire une question de société? On ne peut que partager le constat de l'accélération des disparitions d'un grand nombre d'intellectuels, et précisément des générations de chercheurs qui ont construit leur carrière, leur pensée en constituant des bibliothèques de travail d'avant l'ère du numérique. Certes, l'usage de la lecture en ligne a changé les pratiques d'apprentissage et les bibliothèques privées d'érudits sont peut-être un genre voué à disparaître, encore que le débat reste ouvert. Mais les seuls chiffres, donnés lors de l'ouverture de ce congrès, d'un réseau actif de 2000 sociétés savantes rassemblant 70000 érudits donnent une mesure de la question. La moyenne d'âge y est sans doute plus proche de 75 ans que de 25 . Le phénomène ne peut que s'amplifier, encore sur quelques décennies.

4 Il convient, avant l'exposé, de préciser d'où je parle. Architecte et historienne de formation, je suis fortement intéressée par la question documentaire, un peu écrivain, un peu éditeur, mais non spécialiste, ni bibliothécaire, ni archiviste, fondatrice d'une association d'éducation populaire et de culture partagée, MALTAE, où l'on pratique l'expertise citoyenne et collective dans les domaines de l'architecture, de l'urbanisme, du territoire et du paysage. MALTAE est impliquée dans les sciences de l'éducation, l'histoire et la géographie, fière de se compter parmi les sociétés savantes, inscrite dans l'annuaire des sociétés savantes du CTHS. L'association a une petite activité éditoriale, dont une collection "Lire et écrire le paysage ${ }^{3}$ "; la documentation et la transmission figurent dans son objet social, la question du passage au numérique des ouvrages édités et de son propre fonds est à son ordre du jour. MALTAE était en 2015-2016 partenaire d'un projet pédagogique européen Erasmus +, « Des écrits aux écrans », sur la mutation numérique des apprentissages de l'écriture et de la lecture. Elle y a contribué à plaider la cause de l'alliance entre papier et numérique ${ }^{4}$. La structure compte une quarantaine de membres, dont plusieurs auteurs, un urbaniste chercheur en géographie, Jean-Louis Pacitto, un conseiller pour le livre et la lecture honoraire de la région Provence-AlpesCôte d'Azur, Jean-Claude Gautier, et le responsable du Centre d'archives d'architecture du xx siècle, David Peyceré.

\section{Un panel de douze bibliothèques pour illustrer la diversité des situations}

5 Entre novembre 2017, où j'ai proposé cette communication, et avril 2018, le nombre de cas de bibliothèques pouvant enrichir ma réflexion sur le sujet est passé des deux évoquées ci-dessus à douze. Comment contribuer à garantir un devenir à ces bibliothèques privées, comment les transmettre, en partager la connaissance et en faire un bien commun; faut-il les "patrimonialiser»? En premier lieu, quoi transmettre? Parle-t-on seulement des ouvrages ou des lieux, pièces de travail et de recherche, avec leurs ambiances propices à la réflexion? Peut-on faire passer dans cette transmission, au-delà des ouvrages, une part d'âme de ceux qui les constituèrent? Peut-on y 
retrouver une trace du parcours de l'intellectuel, du chemin de la constitution de sa pensée et de son œuvre? Ces douze cas illustrent une diversité des situations à partir desquelles il est possible de tirer questionnements et pistes de réponses. Je passerai très vite sur les deux bibliothèques par lesquelles je suis entrée dans le sujet pour me pencher sur celles de huit enseignants, chercheurs ou intellectuels avec qui j'ai été en contact, avant de revenir sur les deux bibliothèques de Michel Vernes et Robert Joly, à l'origine de ce propos.

6 Pour procéder par élimination, j'évoquerai en premier lieu ma propre bibliothèque et celle de mon père dont j'héritai il y a cinq ans. L'injonction de mes propres enfants de m'en occuper de mon vivant, sous peine de voir transformer tout ce papier en un grand feu de joie, m'oblige à l'inclure dans le sujet.

\section{Ma bibliothèque}

7 Elle n'est qu'une des potentielles 12 fois 70000 bibliothèques que pourrait connaître tout membre de société savante. Son importance avoisine les 2000 titres; une accumulation, plus qu'une véritable structuration, du fait des déménagements successifs, dont la seule valeur est sans doute de refléter un demi-siècle d'intérêt pour la question du paysage et de témoigner combien elle puise, depuis son émergence, à des champs multiples et éloignés. J'ai donc pleine légitimité à me prendre comme cobaye et à expérimenter la conservation et la transmission de tout ce papier comme bibliothèque virtuelle. Scanners et appareils photo sont déjà disponibles, reste à trouver le temps de procéder à l'expérimentation! Au-delà de la tâche ingrate d'un relevé ou d'un inventaire, au-delà de la tâche matérielle de la numérisation d'une première et d'une quatrième de couverture, éventuellement d'un sommaire, si l'ambition est de dépasser l'austérité d'une simple liste de titres, il s'agit d'en définir les finalités et les modalités. D'une part, de choisir entre un classement structuré, un cheminement chronologique ou une balade dans les livres à créer de toutes pièces; ou de mettre en contexte chaque ouvrage, sa place dans l'ensemble, thématiquement ou chronologiquement (ou les deux, en conciliant trace et usage futur d'un nouveau lecteur, date et conditions de l'arrivée, achat ou cadeau, fruit du hasard ou choix raisonné); ou encore de rouvrir et réveiller chaque livre, en chercher un extrait, l'ajouter comme morceau choisi significatif pour donner à lire l'écriture de l'auteur, extrait qui me parle aujourd'hui ou bien extrait pour refléter l'intérêt porté à l'ouvrage au moment de son entrée dans ma bibliothèque. Et si l'enjeu n'était pas de choisir plutôt un extrait en pensant au futur lecteur, pour lui donner envie? Il s'agit à l'évidence, plus que de transmettre un état existant, de créer de toutes pièces une nouvelle bibliothèque qui intègre à son cahier des charges sa propre transmission! La perspective d'une retraite heureuse était celle de trouver enfin le temps de lire ou relire mes trésors jalousement accumulés, d'y ajouter le projet d'organiser une bibliothèque structurée digne de ce nom, doublée d'une bibliothèque virtuelle et transmissible! L'envie et la motivation ne manquent pas, ni la certitude du plaisir à en retirer. Mais quand vais-je donc trouver le temps de me consacrer à cette nouvelle tâche? Car il s'agit bien d'un nouveau projet, entièrement à concevoir et mettre en œuvre. 


\section{La bibliothèque de mon père}

8 Je passerai aussi rapidement sur le deuxième cas, la bibliothèque de mon père. Pour avoir été ingénieur de 1939 à 1980, après un cursus à l'École navale avant-guerre, sa bibliothèque, qu'il n'aurait peut-être pas osé nommer de son vivant une «bibliothèque d'intellectuel ", était somme toute très ordinaire, modeste, et en volume et en valeur : une coloration personnelle donnée par un demi-siècle d'abonnements à la Revue maritime et à Science et vie. J'ai eu la chance d'avoir assez d'espace pour entasser dans mon grenier les caisses de tout ce qui n'avait pas été beaux livres partagés dans la fratrie. Cinq ans ont passé et cette bibliothèque n'a toujours pas trouvé le début d'un classement, ni le lieu où la valoriser, et encore moins le début d'un temps à lui consacrer pour donner un sens à l'entreprise et répondre au simple enjeu de transmission d'une potentielle bibliothèque virtuelle entre enfants et petits-enfants, projet pourtant aisément accessible avec la numérisation des premières de couverture. Seul le demi-siècle des Science et vie, rangé dans des boîtes en cartons de lait étiquetées à la main et transmis directement à un petit-fils, est conservé dans son intégrité dans une valeur de collection plus affective que vénale. Mais que font les familles où il n'y a ni lieu, ni personne, ni motivation pour cette transmission et que faire pour des bibliothèques dix à vingt fois supérieures en volume?

\section{La bibliothèque gisant sur un trottoir}

Cette question m'a remis en mémoire une autre figure de bibliothèque dont je veux témoigner utilement pour l'avoir connue dans les années 1990 : celle de la bibliothèque gisant sur un trottoir. Nous avons, un soir, été réquisitionnés en tant que propriétaires d'une voiture et à quatre ou cinq nous nous sommes retrouvés à charger, une nuit d'hiver, une bibliothèque d'érudit déversée en piles écroulées, qu'un déménagement d'urgence avait conduite là, lors d'un vidage d'appartement sans ménagement. Je suis de la génération des enfants élevés dans le culte du livre, et le premier choc fut la découverte que c'était donc possible! Le second choc fut de voir la diversité des comportements des personnes appelées en renfort : je m'opposai de manière virulente à ce qu'un tri s'opère, à même le trottoir, autorisant les ramasseurs à décider d'euxmêmes quels livres méritaient d'être sauvés. Charles Maurras et Léon Daudet, qui ne sont pas mes lectures préférées, ont échappé de peu à la benne ; il a fallu convaincre de l'intérêt de conserver sa valeur d'ensemble au fonds récupéré, témoin des lectures d'une époque. L'affaire m'a rappelé celle de la statue du duc d'Anjou, présentée dans l'ouvrage Territoires littéraires, des îles à la ville, Hyères-les-Palmiers, que je publiai la même année sur cinq siècles d'écrits dédiés à la ville d'Hyères, et dont le dessein était justement de faire parler les livres du fonds ancien de la bibliothèque municipale, pour dessiner un portrait de ville. L'architecte Marcel Deslignières fustigeait en 1887 "les républicains qui voulaient abattre la statue de ce tyran sanguinaire, au prétexte qu'elle blessait le sentiment républicain ${ }^{5} »$. Avec un recul de vingt-cinq ans, force est de constater que j'ignore tout de ce qu'il est advenu de l'objet du sauvetage. N'a-t-il pas été vain, s'il ne lui a garanti ni pérennité, ni transmission, sous quelque forme soit-elle? 


\section{La bibliothèque essentielle}

10 La quatrième bibliothèque était géographiquement située dans la même rue, à moins de 300 mètres de cette malheureuse précédente, mais sa destinée fut tout autre. Étienne Juillard, éminent historien et géographe, identifié comme le père de la géographie appliquée, auteur du troisième volume de l'Histoire de la France rurale consacré au XIX ${ }^{e}$ siècle, et qui fut le président d'honneur de MALTAE à ses débuts, m'avait confié, à plus de 90 ans, avoir «fait du tri pour ne garder que l'essentiel, de l'ordre d'une étagère ». Il n'avait gardé que ce qui lui semblait mériter être relu et qu'il relisait, d'ailleurs! Une manière comme une autre de se soucier de son vivant de la transmission de sa bibliothèque, d'en extraire l'essence... J'ignore ce qu'Étienne Juillard a fait des ouvrages qu'il a possédés, et comment il a choisi de s'en séparer, mais un écrit demeure qui illustre sa bibliothèque : un article de 1976 du chercheur Robert Specklin qui fait revivre les débuts de sa carrière d'enseignant et le souvenir dans les années d'après-guerre de sa bibliothèque de travail située à son domicile : "Probablement la seule bibliothèque à peu près complète qui existait alors à Strasbourg sur la géographie de la France ${ }^{6}$." Avec cet article, nous avons hérité de lui un fichier, composé de centaines de petites fiches en carton, remplies au crayon de papier d'une écriture d'un homme du XIXe siècle, fine et penchée. Ce fichier renseigne précisément la production en articles et ouvrages de cet écrivain enseignant-chercheur ; il permet de rappeler que transmettre une bibliothèque d'un enseignant-chercheur ou intellectuel inclut de mentionner ses propres écrits, ouvrages et articles. Conserver trace de son œuvre d'intellectuel et la contextualiser, si possible, parmi celles de ses pairs, est à l'évidence une tâche à accomplir de son vivant ; c'est sans doute une des finalités à attendre d'une transmission de bibliothèque.

\section{Un chantier en cours de bibliothèque virtuelle}

11 Telle a été la démarche de cet autre historien, Maurice Agulhon, grâce à un projet porté par l'université d'Avignon, comprenant le financement d'un inventaire et celui de la réalisation d'un film permettant une visite virtuelle de la bibliothèque. L'article de Jonathan Barbier et Nathalie Petiteau ${ }^{7}$ insiste sur la sensibilisation de l'enseignantchercheur à la question du devenir de sa bibliothèque de travail, afin de la rendre utile aux chercheurs. Maurice Agulhon a donc, de son vivant, réorganisé sa bibliothèque du point de vue de son utilité pour les chercheurs. Légitime et efficace, cet arbitrage a fait disparaître la structuration initiale des collections qui aurait donné à lire le cheminement d'une pensée sur trois quarts de siècle. Si la question de la transmission implique en général l'existence des points de vue à la fois de l'émetteur et du récepteur, le point de vue retenu dans la présente démarche est celui du récepteur, puisque l'objectif $\mathrm{du}$ projet est une appropriation rendue possible via l'ouverture aux chercheurs d'une bibliothèque privée présente dans la demeure familiale. Pourtant, l'article de Jonathan Barbier et Nathalie Petiteau conclut : « Reste que la constitution de la bibliothèque de Villeneuve-lez-Avignon en véritable patrimoine est un problème non résolu. [...] Quant à une donation à l'université d'Avignon qui l'a inventoriée et filmée, elle n'est nullement à l'ordre du jour. » Maurice Agulhon s'y est attelé de son vivant ; un partenariat avec une université prestigieuse a été trouvé pour apporter le financement et l'outillage scientifique, mais la pérennité recherchée n'est pas encore au bout du chemin! 


\section{Un champ des possibles : une transmission de bibliothèque à inventer}

12 Jean-Paul Ferrier, décédé en 2016, est lui aussi historien et géographe, professeur d'une autre université de Provence, celle d'Aix. Son épouse ne s'est pas encore résolue à trier les ouvrages. «Rien ne presse, la maison est assez vaste, mais il faudra bien s'y mettre ! Un de nos fils s'intéresse à une partie des livres ; mais il y a tellement de revues... et les "Que sais- je ?", ceux-là on peut bien les éliminer. On a commencé à jeter les travaux d'étudiants, il y en a tellement ! Même l'université ne les veut pas. » Peut-il y avoir une autre réponse qu'un inventaire exhaustif et préalable à tout tri ? Si plus rien ne peut être fait du vivant de Jean-Paul Ferrier, le réseau encore actif constitué par un groupe de collègues permettrait de faire parler les livres. Amis ou collègues auraient chacun certainement quelque chose à dire d'un ouvrage spécifique que, dans sa carrière d'enseignant-chercheur, Jean-Paul Ferrier aurait jugé précieux ou qu'il aurait écrit. Le devenir de sa bibliothèque privée demeure dans le champ des possibles, entre les mains de sa famille et de ses pairs ou amis. Rien n'a bougé, ni des étagères du salon, dédiées aux livres précieux et anciens de géographie, ni la bibliothèque de la pièce de travail, ni le stockage en haut de la mezzanine (dont les «Que sais-je?»), ni les étagères de la chambre consacrées aux ouvrages littéraires, lectures communes avec son épouse. Les allers-retours en vue de réaliser la présente contribution aideront peut-être à déclencher un projet: tout reste à imaginer, mais il apparaît clairement que la transmission des bibliothèques de travail des enseignants-chercheurs ne peut pas reposer sur les seules épaules des familles.

\section{La bibliothèque éclatée : « trop tard » ou « il est encore temps » ?}

En 2011, Robert Joly et Marcel Roncayolo avaient engagé des entretiens croisés, auxquels ils m'avaient associée; leur projet était de mutualiser leurs regards d'urbaniste et de géographe pour " refaire la ville », projet éponyme de l'ouvrage écrit par Robert Joly en 1996. Le décès de Robert Joly a interrompu le projet; mon intérêt éveillé par les cas des bibliothèques de Michel Vernes et de Robert Joly m'a conduite à proposer à la fille de Marcel Roncayolo de réorienter le sujet de ces entretiens autour de la constitution de sa bibliothèque de travail, en invitant l'éminent géographe et historien de Marseille à parler de ses lectures fondatrices et de ses livres fétiches. Malheureusement, l'éclatement de sa bibliothèque privée sur ses deux lieux d'habitation de Marseille et Paris a rendu l'entreprise impossible: difficile de faire parler les livres, quand ils sont matériellement éloignés de 800 kilomètres! Ce septième témoignage peut laisser penser qu'il est trop tard pour reconstituer la bibliothèque, à moins qu'il soit encore possible de dire qu'il est encore temps, mais les modalités doivent être rapidement trouvées !

\section{Les chantiers à ouvrir : des bibliothèques à transmettre de leur « vivant »}

Jean-Pierre Frey, urbaniste et sociologue, proche de Marcel Roncayolo pour lui avoir succédé à la direction de l'Institut d'urbanisme de Paris, a été sollicité pour participer à 
ce projet de faire avec Marcel Roncayolo le tour du propriétaire de sa bibliothèque. Immédiatement, Jean-Pierre Frey, alors jeune retraité de sa carrière d'enseignant, a évoqué sa propre bibliothèque de plus de 5000 titres et le problème que son devenir lui posait. Par ailleurs, quand j'ai voulu prendre des nouvelles de la bibliothèque de Michel Vernes, j'ai cherché à joindre Bertrand Lemoine, qui avait été directeur de l'École nationale supérieure d'architecture de Paris-La Villette où Michel Vernes avait fait sa carrière d'enseignant. Immédiatement, Bertrand Lemoine a lui aussi évoqué sa propre bibliothèque de plus de 5000 titres et le problème que son devenir lui posait. Ces cas désignent, à l'évidence, une catégorie de bibliothèques de même volume en mal de devenir, mais, plus encore, une catégorie d'acteurs, chercheurs, érudits dans la force de l'âge. Ces acteurs conscients de la nécessité et de l'urgence de l'entreprise de transmission et, qui plus est, en pleine capacité intellectuelle et potentiellement disponibles pour s'atteler à la tâche sont ceux qu'il convient de mobiliser pour constituer un groupe de travail ad hoc, en situation de recherche-action.

Jean-Claude Gautier eut à s'occuper, en qualité de conseiller pour le livre et la lecture à la direction régionale des affaires culturelles Provence-Alpes-Côte d'Azur, de la construction de nombreuses bibliothèques publiques. Nous nous sommes retrouvés à Marseille, justement lors de la présentation du dernier ouvrage de Marcel Roncayolo, Le géographe dans sa ville (Parenthèses, 2016). Par goût de son métier, il s'est constitué sa vie durant une belle bibliothèque et appartient lui aussi à cette catégorie d'intellectuels dans la force de l'âge, conscients de l'urgence et de la nécessité de l'entreprise de transmission ${ }^{8}$. L'évolution du périmètre de sa bibliothèque, dont il nous donne des nouvelles de temps à autre, montre que cette tâche est déjà largement amorcée, de par les contraintes d'un déménagement d'abord, puis méticuleusement opérée au fil de l'eau par l'intéressé lui-même. Il a profité du réseau constitué durant sa vie professionnelle pour procéder à des donations d'ensembles thématiques dans les établissements de lecture publique les plus appropriés. C'est un projet qui procède par dépossessions successives et raisonnées. Jean-Claude Gautier ne nous a pas informé s'il s'est intéressé à en garder la trace.

\section{La démesure : la bibliothèque-appartement « piles de livres » de Michel Vernes}

Évoquer le sauvetage de la bibliothèque de Michel Vernes conduit à passer dans la cour des grands, changer d'échelle; elle mériterait une étude à elle seule et de laisser conter cette histoire par Marc Le Cœur, historien de l'architecture lui-même et neveu de Michel Vernes, qui assuma la direction des opérations 9 . Pour mesurer l'épopée qui s'engagea avec un tel héritage, ce fut, selon ses dires, "trois ans de vie, à mettre 30000 livres en 800 caisses », pour réaliser finalement ce qu'il propose de nommer une bibliothèque posthume réorganisée par le réceptionnaire en trente-deux thématiques ${ }^{10}$. Les piles de livres emplissaient l'appartement jusqu'à ne plus pouvoir y tenir! Son épouse disait qu'il n'y avait pas un jour sans qu'il en achète un ou plusieurs. Lorsqu'il devait rechercher une référence dans ces empilements non classés, il lui était plus rapide de la racheter, augmentant d'autant de doublons le nombre d'ouvrages! L'École nationale supérieure d'architecture de Paris-La Villette a finalement reçu le fonds, mais, à ce jour, il n'est toujours qu'un stock de 800 caisses de livres... On est donc passé, en cinq ans, d'un appartement privé "piles de livres" à un fonds semi-public de «caisses de livres» déposé dans un établissement d'enseignement public. Quid de la 
valorisation et de la transmission du parcours d'intellectuel ? Le chemin semble encore long!

Ce cas donne une mesure tout d'abord de ma naïveté première, mais surtout de l'étendue des chantiers à ouvrir et à conduire pour faire parler ces livres, si tant est qu'on y trouverait un sens ! Comment donner à cette bibliothèque un devenir, associant réel et virtuel, qui inclurait cette démarche de rendre compte du parcours d'intellectuel de l'enseignant-chercheur. Néanmoins, un travail colossal a déjà été entrepris puisque Marc Le Cœur, lors du déménagement de l'appartement, a procédé à un repérage photographique systématique des ouvrages au fur et à mesure de leur mise en caisse, constituant la matière première d'un inventaire ou d'un relevé. Par-delà cette étape, un classement en a même déjà été fait, construit a posteriori, condition d'une potentielle accessibilité, même si celle-ci implique un lieu de stockage associé à une bibliothèque ou un centre d'archives... Une des hypothèses serait de déposer cette bibliothèque dans les fonds des Archives de la critique d'art (ACA), constituées en 1989 en association, puis groupement d'intérêt scientifique rattaché à l'université de Rennes, en espérant y trouver des ressources en personnel pour son traitement. Le prix à payer serait une séparation des livres du fonds d'archives, lui-même déposé à l'École nationale supérieure d'architecture de Paris-La Villette, au risque d'éloigner le fonds de ceux qui devraient en être les premiers intéressés en tant qu'usagers, enseignantschercheurs et étudiants de cette école. Arbitrage qui implique de poser la question du "pour qui »?

On peut imaginer que, depuis l'École nationale supérieure d'architecture de Paris-La Villette, il est possible de retrouver bon nombre des étudiants de Michel Vernes. Des entretiens pourraient faire parler certains de ces 30000 livres et enrichir le portrait d'un homme d'exception. Ces nouvelles archives orales, à créer, contribueraient à la constitution d'une mémoire de l'enseignement de ce professeur. Peut-on imaginer un tel investissement de temps et d'argent pour chaque enseignant de chaque école? Sûrement non! Mais le fondateur d'une école, à la bibliothèque si notoire, mériterait peut-être un traitement d'exception.

Il semblerait qu'en matière de conservation d'archives de lieux d'enseignement le versement des fonds administratifs soit privilégié aux archives elles-mêmes et aux bibliothèques des chercheurs. Affaire à suivre; cette valorisation est loin d'être achevée, mais elle est placée entre les mains de personnes et d'institutions autorisées et compétentes. Nous pouvons ici en tirer comme enseignement d'une part qu'une sensibilisation des institutions est encore à conduire, et d'autre part qu'une transmission aboutie doit concilier pérennité du transfert, accès aux ouvrages et mobilisation de moyens en personnel pour son traitement.

\section{Une transmission de bibliothèque documentée, inventoriée et intégrée à l'activité de recherche}

Passons à la bibliothèque de travail de Robert Joly, enseignant-chercheur, praticien et théoricien, longtemps enseignant à l'École nationale supérieure d'architecture de ParisLa Villette. Je commencerai par évoquer l'homme que j'ai connu comme enseignant puis comme patron dans une relation de maître à élève qui a évolué sur trente ans, jusqu'à devenir une relation de pairs et d'amis. En 2010, il devient sujet et objet d'étude de mon activité d'historienne de l'architecture dans le film que j'engage avec Christian 
Girier et Jean-Louis Pacitto, Retour sur site. Un maître d'œuvre et son ouvrage : Robert Joly et le lycée agricole de Tulle-Naves ${ }^{11}$ (fig. 1). Son écriture avait à voir avec celle d'Étienne Juillard, dans le soin, la finesse et l'usage du crayon-mine. Cette remarque vaut pour souligner la place des écrits et de l'écriture dans les bibliothèques d'intellectuels, dont les annotations en marge et les notes de travail encore en place dans les ouvrages restent la source la plus précieuse pour suivre la pensée en train de s'élaborer : cette transmission où le maître, en situation d'élève, apprend d'un autre maître, qu'il en soit le pair ou non (fig. 2).

Fig. 1. - Robert Joly, un an avant sa mort, devant le lycée agricole de Tulle-Naves, l'édifice en béton brut qu'il construisit en 1971.

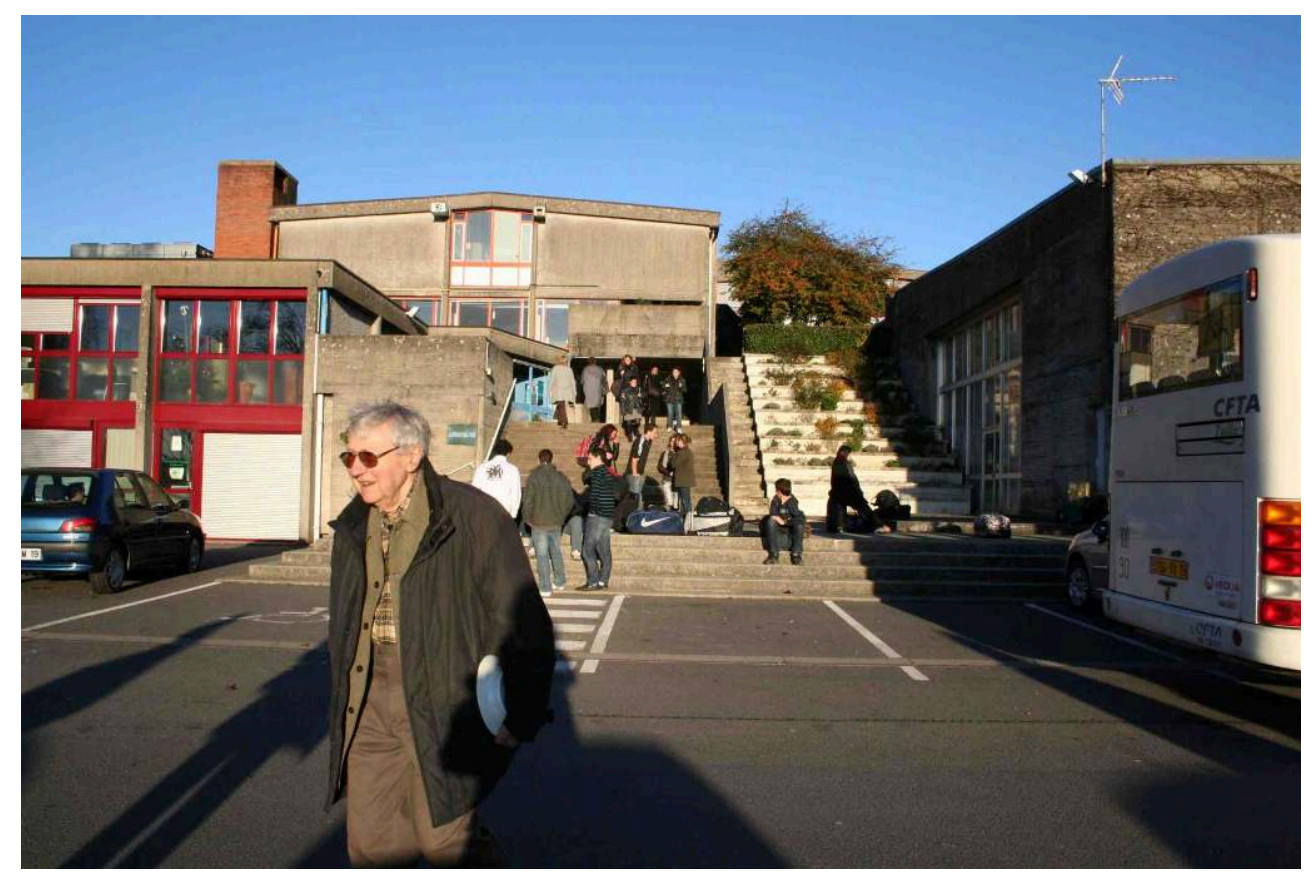

Extrait de C. Girier, O. Jacquemin, J.-L. Pacitto, Retour sur site. Un maitre d'œuvre et son ouvrage : Robert Joly et le lycée agricole de Tulle-Naves, film documentaire, MALTAE, 2011.

D.R. 
Fig. 2. - Bibliothèque de Robert Joly. Note de lecture, traces d'une pensée en cours d'élaboration.

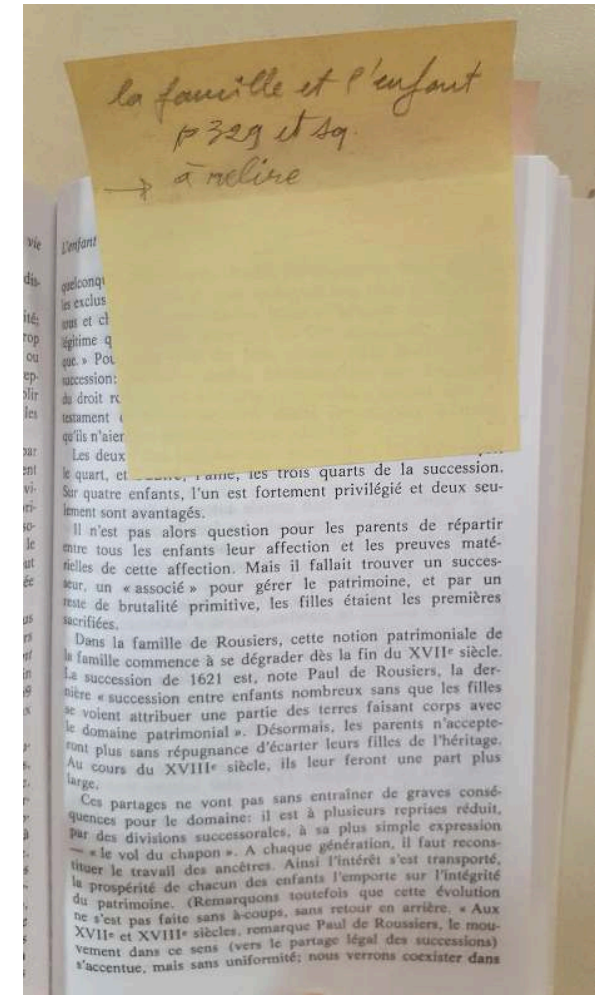

Cliché O. Jacquemin.

21 Au-delà du choix des titres et des auteurs des ouvrages retenus pour leur contenu, le tri opéré par David Peyceré lors de la réception du fonds d'archives au Centre d'archives d'architecture $d u x^{e}$ siècle après le décès de Robert Joly (2013) a notamment été motivé par la capacité de ces références à parler de Robert Joly, à travers leurs annotations, commentaires ou marque-pages déposés. Ils renseignent aussi sur l'état d'âme de l'homme, la part du sensible (fig. 3). À la suite de ce versement aux archives de l'architecture, le reliquat vint constituer la part de bibliothèque dont MALTAE a hérité de la famille. 
Fig. 3. - Bibliothèque de Robert Joly. La part du sensible : feuille d'automne, marque-page trouvé en revenant du Louvre.

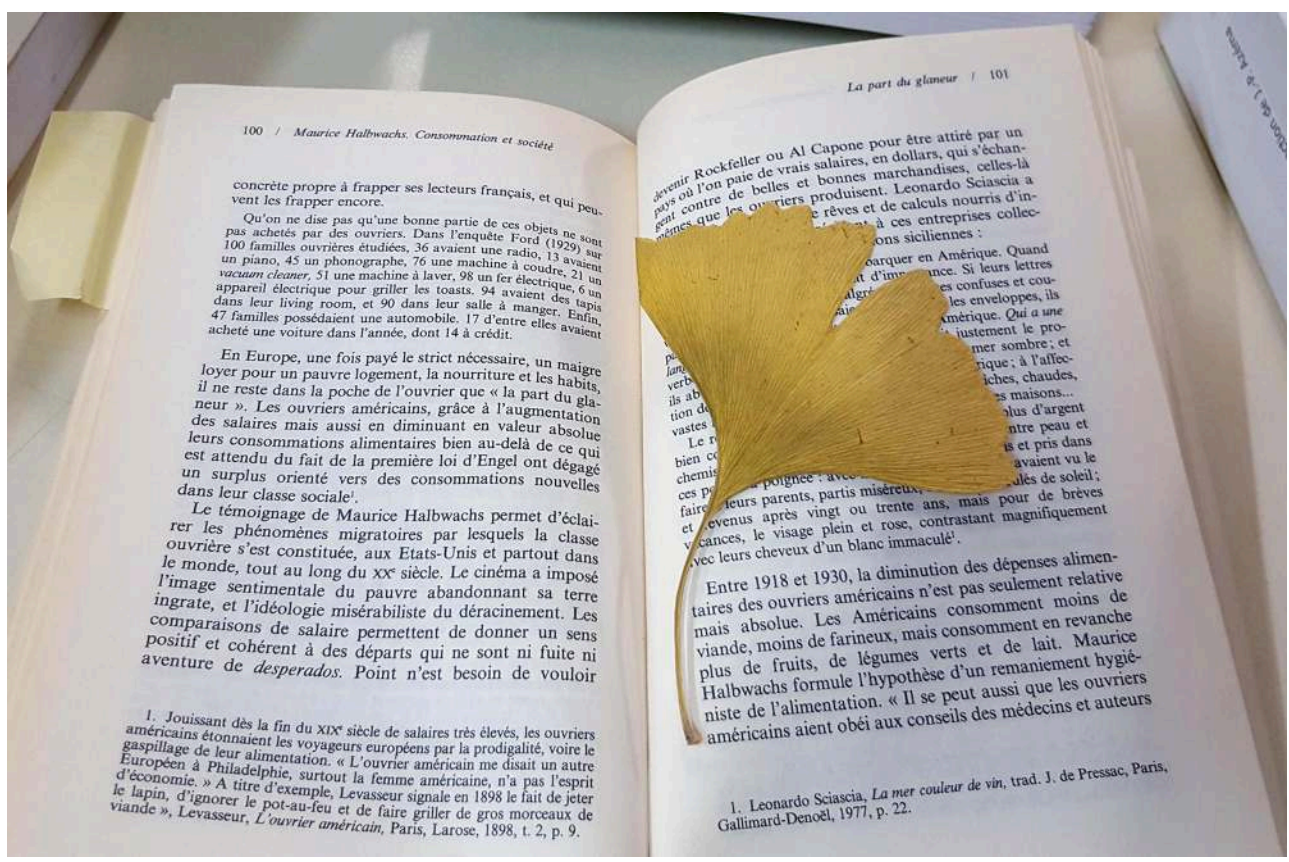

Cliché O. Jacquemin

Ce fut la grande chance de cette bibliothèque privée, ce que confirment les trois filles de Robert Joly, Hélène, Anne et Françoise, interrogées pour cette contribution. Le dépôt des archives professionnelles de celui-ci, huit ans auparavant, de son vivant, avait tracé la voie et permis de dédier à l'étude de l'œuvre protéiforme de cet architecte atypique, urbaniste, chercheur, théoricien et enseignant, une thèse de doctorat. Alexandra Schlicklin avait pu fréquenter et interviewer Robert Joly, dépouiller ses archives avant de procéder, à son décès, au relevé de sa bibliothèque, en respectant le classement thématique des étagères, rigoureusement photographiées. «La bibliothèque était constituée pour partie des livres personnels de Robert Joly, et pour partie du fonds de livres du Groupement des architectes associés $\left(\mathrm{GAA}^{12}\right)$, car l'agence possédait sa propre bibliothèque documentaire. Les relever, même succinctement, mais avec une méthode exploitable, a nécessité au total une semaine de travail. Le relevé en est présenté dans les annexes de la thèse et en est resté volontairement à la logique, d'ailleurs déjà thématisée, du classement des étagères et des pièces accueillant les livres ${ }^{13}$. » Une fois les caisses de livres déposées rue de Tolbiac, David Peyceré en fit faire un classement alphabétique par auteur et, par simple surlignage, la mention de ceux entrés dans le fonds classé. La présence des enfants de l'architecte permet encore de faire parler les livres, ponctuellement, tout au moins en lien avec la dimension d'histoire familiale que revêt une bibliothèque privée. Anne évoque des lectures partagées autour de Gaston Bachelard, qui donna lieu à un projet commun que le décès de son père ne permit pas de mettre en œuvre. Françoise se souvient plus douloureusement du poids de l'injonction à lire que représentait cette vie construite autour des livres, où ils avaient tant d'importance dans ce couple d'intellectuels, chacun dans sa spécialité ! « Comment être à la hauteur, quand on est petit et que la barre est si haut?» Pour Hélène, la bibliothèque témoigne d'une culture du monde des lettres et, au-delà des livres, « de l'amour du verbe juste » qui a été transmis dans la maison familiale, jusque dans la 
parole. La thèse d'Alexandra Schlicklin, en liant le relevé de la bibliothèque au dépouillement des archives, aux entretiens oraux conduits du vivant de Robert Joly et autres médias ${ }^{14}$ au titre de ses sources, répond en partie aux défis que nous posions ici en introduction sur les enjeux des transmissions des bibliothèques d'érudits. Sa thèse se veut à la fois biographie de l'auteur et monographie sur son œuvre; en revanche, la bibliothèque y a perdu son statut de corpus autonome. Faire parler ses livres pour retracer le parcours intellectuel et dresser le portrait de Robert Joly par et dans sa bibliothèque est un projet qui aujourd'hui serait vain, sauf à inclure la thèse d'Alexandra Schlicklin au rang des sources susceptibles d'éclairer un portrait posthume qui pourrait être fait de lui complété d'une balade littéraire dans sa bibliothèque !

\section{Questionnements et pistes}

Aux questionnements énumérés dans ce tour d'horizon, deux pistes peuvent être retenues afin de répondre à plusieurs cas de figures.

La première piste implique certaines conditions simples mais exigeantes qui permettraient de réagir au cas par cas. Il s'agit notamment de prendre conscience que ces opérations exigent temps et motivation; de garantir une bienveillance et une disponibilité de la part de l'entourage familial; de pouvoir bénéficier d'un minimum de formation pour la rigueur des relevés et des classements; de s'assurer de conditions techniques de numérisation et de transfert matériel et immatériel satisfaisantes; de pouvoir s'assurer du soutien, voire du partenariat d'institutions publiques concernées ; de garantir la pérennité des solutions proposées; de réunir les conditions permettant de mener ces actions autant que possible avec leur propriétaire, de les monter de leur vivant.

Une seconde piste, plus ambitieuse, serait de répondre à la question de la préservation de ces bibliothèques en leur qualité de patrimoine scientifique et culturel pour les générations futures. Le défi est alors d'assurer la transformation de patrimoines privés matériels en un bien commun immatériel. Il s'agirait tout d'abord d'impliquer le plus en amont possible les intellectuels concernés pour qu'ils s'occupent de leur vivant de leur bibliothèque. Il est nécessaire de faire prendre conscience que transmettre une bibliothèque est un prolongement indissociable et inévitable de l'acte de constituer cette bibliothèque; et notamment en $\mathrm{y}$ intégrant la propre production de son propriétaire, en la contextualisant parmi celle de ses pairs. Réussir la transmission du cheminement, de l'élaboration d'une pensée et d'un savoir est l'un de ces enjeux, qui nécessite leur collaboration, même s'il est toujours possible de reconstituer une bibliothèque posthume comme la nomme Marc Le Cœur ${ }^{15}$. Il conviendrait également de mettre un pluriel à ces transmissions, de leur reconnaître le statut de projets culturels à part entière, la part de créativité qui leur est inhérente. Enfin il paraît nécessaire de faire prendre conscience aux institutions des enjeux scientifiques et du caractère de bien commun que représente cette transmission, autant que de la nécessité de sortir les familles de leur isolement face à cette problématique et face à une tâche qui les dépasse, souvent dans une période délicate de deuil et de règlement de succession.

Face à l'ampleur de la question, le CTHS pourrait mobiliser les sociétés savantes pour qu'elles partagent responsabilités et initiatives avec les familles, pour qu'elles exercent une veille sur les bibliothèques les plus urgentes à sauvegarder, par exemple en lançant une enquête en vue d'un premier état des lieux des bibliothèques concernées. 
27 De même, il s'agirait d'engager une dynamique collaborative, de constituer un groupe de travail ad hoc à travers lequel pourraient être mobilisés les jeunes retraités concernés par le devenir de leur bibliothèque et de jeunes étudiants prêts à s'impliquer en situation de recherche-action ${ }^{16}$.

Enfin, au-delà, il conviendrait d'impliquer la société civile et de faire de la transmission des bibliothèques de savants des chantiers de sciences participatives: faire du transgénérationnel appliqué au domaine de l'érudition... De développer des compétences de passeurs de culture et de multiplier les occasions de passage à l'acte. Pour les plus jeunes, ce seraient des occasions privilégiées de côtoyer des érudits d'un genre en voie de disparition et, pour les moins jeunes qui acceptent de préparer et d'accompagner la transmission de leur bibliothèque, ce seraient des occasions privilégiées de revenir sur leur carrière, de faire un bilan de leur vie professionnelle, des occasions uniques de laisser une archive orale du type de la balade littéraire ou du dialogue avec leurs livres, que nul autre ne pourrait reconstituer à leur place, de prolonger leur pratique de l'enseignement dans un dernier exercice de style, pour répondre au défi de donner envie, de s'inviter enfin à coproduire une œuvre de création, placée entre la relecture de l'un et la découverte de l'autre, de donner sens à une rencontre improbable...

Certes, il convient de voir la part d'utopie dans l'idée de ces 800000 potentielles bibliothèques à transmettre, en y faisant parler les livres. Il faut sans doute accepter qu'un tri s'opère, qu'une part d'abandon inhérente au principe de conservation existe, qu'une part d'aléatoire se réalise, que la nouvelle vie que représente un livre donné à un proche a aussi sa valeur puisque, à son tour, il y trouvera peut-être un texte fondateur pour nourrir sa propre pensée, à quarante ans et 200 pages de distance de premier lecteur qui l'avait annoté...

30 Toutefois, il faut faire savoir que transmettre est un vrai projet ! Ce qui est valable pour la transmission d'une terre cultivée en biodynamie depuis trente ans l'est tout autant pour une bibliothèque soigneusement et amoureusement constituée pendant soixante ans. Dans les deux cas, le temps du projet est au minimum une affaire de dix ans! C'est un chantier ouvert qui implique, pour prolonger la comparaison avec la terre, de remettre du vivant, de l'humain au cœur des livres, des caisses et des étagères, en bref des lecteurs!

31 Faire d'une bibliothèque à transmettre un patrimoine vivant, en devenir, implique de développer une culture du projet et les compétences de créativité nécessaires à sa mise en œuvre. Cette reconnaissance d'un projet culturel créatif à partager n'est-elle pas le moyen de répondre à la part douloureuse de dépossession contenue dans la transmission et de garantir qu'il y a bien eu héritage?

David Peyceré trouvait à un tel projet un air de bibliothèque vertigineuse, s'apparentant à la mise en abîme chère à Jorge Luis Borges et développée dans $L a$ bibliothèque de Babel en 1941. De la bibliothèque au livre, au titre, au mot, de la bibliothèque au catalogue, un catalogue plus important que la bibliothèque puisqu'il la contient toute et y compris le lecteur... Pour donner le mot de la fin à l'écrivain Borges et à Hélène Joly, pour qui l'essence de la transmission de la bibliothèque familiale est dans le goût transmis du "verbe juste ", ce plaidoyer pour faire parler les livres d'une 
bibliothèque se conclura sur le vœu que les transmissions remettent parole et langage au cœur des projets, dans un échange ouvrant le passage de l'écrit à l'oral, quelles qu'en soient les formes multiples.

\section{BIBLIOGRAPHIE}

BARBIER Jonathan, PETITEAU Natalie, « La bibliothèque de Maurice Agulhon : cadre de travail, coulisses de l'écriture et patrimoine scientifique ", Revue d'histoire du XIX $x^{e}$ siècle, éd. numérique, $\mathrm{n}^{\circ} 47,2013$. [URL : https://journals.openedition.org/rh19/4565]

Des écrits aux écrans : livre blanc, éd . numérique, projet Erasmus +, [s. 1.], 2015. [URL : http:// www.scriptanumerica.eu]

JACQUEMIN Odile, BERRO Catherine, DAUGA Hélène, Territoires littéraires, des îles à la ville, Hyères-lesPalmiers : écrits d'une ville, Hyères, Mémoire à lire, territoire à l'écoute, 1998.

JUILLARD Étienne, Le Var et les Maures, entre histoire et géographie : recueil d'articles (1957-2006), Hyères, Mémoire à lire, territoire à l'écoute, 2015.

LE CEUUR Marc, LEMOINE Bertrand, « Hommage à Michel Vernes », Archicréée, n 360, 2013, p. 28-30. SCHLICKLIN Alexandra, « Robert Joly (1928-2012), urbaniste, moderne, intellectuel : les nouvelles figures de l'architecte », thèse de doctorat, Metz, université de Lorraine, 2014.

\section{NOTES}

1. Créée il y a vingt-cinq ans à Hyères dans le Var, MALTAE a pour objet la culture du territoire partagé par la valorisation du patrimoine architectural, urbain, paysager et environnemental. Association d'éducation populaire, dans une posture entre sciences et arts, elle pratique l'animation territoriale, avec des conférences, des expositions, des visites et débats, elle édite et produit des films d'archives.

2. Centre d'archives d'architecture du $\mathrm{xx}^{\mathrm{e}}$ siècle, IFA cote 38 , fonds Robert Joly (1928-2012).

3. Les trois axes de son projet éditorial sont l'exploration du temps (de ses différentes échelles, du quotidien, de l'histoire et de la prospective), celle du paysage habité (derrière les lieux, les hommes qui les ont produits et qui y vivent) et celle d'une approche anthropologique qui se refuse à dissocier nature et culture: archives textuelles, photographiques ou orales et œuvres contemporaines déclinent une approche sensible du territoire entre arts et sciences. Parmi les titres, rééditions d'introuvables, hommages à des lettrés disparus, publications de thèses, romans, traductions de rapports de recherche et études en papier gris... : Odile Jacquemin, Deux siècles d'histoire d'un paysage entre terre et mer: Hyères de 1748 à nos jours, 2012; Christian Dental, Le petit jardinier, 2011 ; Odile Jacquemin et Jean Louis Pacitto (dir.), Jean Belvisi (photographies), Traits de côte, arpents de mer: carnet de bord d'une expérimentation de 
gestion intégrée des zones côtières, 2008; Pierre Foncin, Odile Jacquemin (préf.), Jean Belvisi (photographies), Les Maures et l'Esterel, rééd., 2008; George Sand, Nathalie Bertrand (préf.), Hélène Dauga (ill.), Tamaris, rééd., 2008.

4. Des écrits aux écrans : livre blanc.

5. O. Jacquemin, C. Berro, H. Dauga, Territoires littéraires, des îles à la ville, Hyères-lesPalmiers : écrits d'une ville, p. 178.

6. R. Specklin, dans É. Juillard, Le Var et les Maures, entre histoire et géographie: recueil d'articles (1957-2006), p. 24.

7. J. Barbier, N. Petiteau, «La bibliothèque de Maurice Agulhon: cadre de travail, coulisses de l'écriture et patrimoine scientifique ».

8. Il contribua activement au ${ }^{\circ} 74$ des Études drômoises, paru en 2018 et consacré aux passeurs de culture européens installés dans la Drôme.

9. M. Le Cœur, B. Lemoine, « Hommage à Michel Vernes ».

10. À titre de repère, pour donner une idée de ce que peut représenter un appartement rempli de 30000 volumes, la bibliothèque de Jacques Doucet, constituée de 90 fonds de grands auteurs, tels Charles Baudelaire ou René Char, rassemble 50000 ouvrages (et 140000 manuscrits).

11. C. Girier, O. Jacquemin, J.-L. Pacitto, Retour sur site. Un maitre d'œuvre et son ouvrage: Robert Joly et le lycée agricole de Tulle-Naves, film documentaire, MALTAE, 2011.

12. Agence fondée avec Robert Joly et Simone Mourot en 1963.

13. A. Schlicklin, «Robert Joly (1928-2012), urbaniste, moderne, intellectuel: les nouvelles figures de l'architecte », p. 23.

14. A. Schlicklin introduit sa thèse avec une citation de trois jeunes internes, filmés en situation de réception de l'œuvre: «C'est notre maison!» (Dans C.Girier, O. Jacquemin, J.-L. Pacitto, Retour sur site. Un maître d'œuvre et son ouvrage : Robert Joly et le lycée agricole de Tulle-Naves, film documentaire, MALTAE, 2011.)

15. M. Le Cœur, B. Lemoine, « Hommage à Michel Vernes ».

16. «Donner envie à des jeunes»: cette injonction nous a fait tester en 2020 un protocole de péesentation d'ouvrage, à mi-chemin entre le sérieux de la notice bibliographique et le coup de cœur du libraire ou du blog de lecteur pour la réalisation d'une bibliothèque virtuelle du projet éducatif Erasmus + "Cooper'actif: habiter ensemble autrement demain » (http://habitat-cooperactif.eu/)

\section{RÉSUMÉS}

Avec la dispersion de la bibliothèque d'un enseignant à son décès, c'est tout un pan de connaissance sur le savoir constitué qui disparaît. La bibliothèque privée d'intellectuel a un double statut de patrimoine matériel et immatériel, elle est la trace du cheminement de sa pensée, du contexte et du cadre culturel de l'élaboration du savoir en jeu. L'ère du numérique et des bibliothèques virtuelles permet aujourd'hui de transmettre la valeur d'ensemble de la 
bibliothèque et de son contenu, indépendamment des questions d'héritage, de succession liées à la valeur vénale des ouvrages. Historienne et architecte, l'auteure a participé aux débats sur le devenir de deux bibliothèques privées de deux de ses enseignants, fondateurs de l'École nationale supérieure d'architecture de Paris-La Villette, alors UP6, Robert Joly et Michel Vernes, décédés en 2012 et 2013. Cette contribution propose, en partant de ces deux exemples, vite devenus douze, un plaidoyer et un apport méthodologique.

\section{AUTEUR}

\section{ODILE JACQUEMIN}

Docteur en histoire culturelle

Architecte urbaniste

Directrice de l'association MALTAE (Mémoire à lire, territoire à l'écoute) 